Article

\title{
The $R^{r}$ Form of the Kedem-Katchalsky-Peusner Model Equations for Description of the Membrane Transport in Concentration Polarization Conditions
}

\author{
Kornelia M. Batko ${ }^{1, *}$ C , Andrzej Ślęzak ${ }^{2}$, Sławomir Grzegorczyn ${ }^{3}$ and Wioletta M. Bajdur ${ }^{2, *}$ \\ 1 Department of Business Informatics, University of Economics, 40287 Katowice, Poland \\ 2 Department of Innovation and Safety Management Systems, Technical University of Czestochowa, \\ 42200 Czestochowa, Poland; aslezak52@gmail.com \\ 3 Department of Biophysics, Faculty of Medicine with the Division of Dentistry in Zabrze, Medical University \\ of Silesia, 19 H. Jordan Str., 41808 Zabrze, Poland; grzegorczyn@sum.edu.pl \\ * Correspondence: kornelia.batko@ue.katowice.pl (K.M.B.); wiolawb@poczta.onet.pl (W.M.B.)
}

Received: 3 July 2020; Accepted: 30 July 2020; Published: 1 August 2020

check for updates

\begin{abstract}
The paper presents the $R^{r}$ matrix form of Kedem-Katchalsky-Peusner equations for membrane transport of the non-homogeneous ternary non-electrolyte solutions. Peusner's coefficients $R_{i j}^{r}$ and $\operatorname{det}\left[R^{r}\right](i, j \in\{1,2,3\}, r=\mathrm{A}, \mathrm{B})$ occurring in these equations, were calculated for Nephrophan biomembrane, glucose in aqueous ethanol solutions and two different settings of the solutions relative to the horizontally oriented membrane for concentration polarization conditions or homogeneity of solutions. Kedem-Katchalsky coefficients, measured for homogeneous and non-homogeneous solutions, were used for the calculations. The calculated Peusner's coefficients for homogeneous solutions depend linearly, and for non-homogeneous solutions non-linearly on the concentrations of solutes. The concentration dependences of the coefficients $R_{i j}^{r}$ and $\operatorname{det}\left[R^{r}\right]$ indicate a characteristic glucose concentration of $9.24 \mathrm{~mol} / \mathrm{m}^{3}$ (at a fixed ethanol concentration) in which the obtained curves for Configurations A and B intersect. At this point, the density of solutions in the upper and lower membrane chamber are the same. Peusner's coefficients were used to assess the effect of concentration polarization and free convection on membrane transport (the $\xi_{i j}$ coefficient), determine the degree of coupling (the $r_{i j}^{r}$ coefficient) and coupling parameter (the $Q_{R}^{r}$ coefficient) and energy conversion efficiency (the $\left(e_{i j}^{r}\right)_{r}$ coefficient).
\end{abstract}

Keywords: membrane transport; non-electrolyte solutions; Peusner's network thermodynamics; Kedem-Katchalsky equations; concentration polarization

\section{Introduction}

Membrane transport belongs to the group of processes described by thermodynamics of irreversible processes, now called modern thermodynamics. This theory was created and described by Lars Onsager, Theophile De Donder, Ilya Prigogine and others [1]. This field of knowledge has provided many research tools for transport mechanisms, including membrane transport, which is used in many areas of science (physics, biology, chemistry) and technology (biotechnology, biomedical engineering, water and sewage engineering, bioenergetics) [2-9]. One of the basic research tools for membrane transport are the Kedem-Katchalsky Equations (K-K Equations) derived from Onsager thermodynamics. The $\mathrm{K}-\mathrm{K}$ Equations show the relationship between volume $\left(J_{v}\right)$, solute $\left(J_{s}\right)$ fluxes and thermodynamic forces (osmotic $\Delta \pi$ and/or hydrostatic $\Delta P$ ) $[10,11]$. Currently, several versions of these equations classical form [12] and forms presented by Kargol and Kargol [13,14], Peusner [15], Elmoazzen et al. [16], Cheng and Pinsky [17] and Cardoso and Cartwright [18]. 
The starting point of Onsager thermodynamics is the scattering function: $\Phi=T\left(d_{i} S / d t\right)$, where $T$ is the absolute temperature, and $d_{i} S / d t$ - the production of internal entropy [8,11]. For isothermal processes: $\Phi=\sum_{i} J_{i} X_{i}$. If the forces $\left(X_{i}\right)$ and flows $\left(J_{i}\right)$ are related by linear equations in the form $X_{i}=\sum_{j} R_{i j} J_{j}$, then the matrix of coefficients $R$ is symmetrical, i.e., $R_{i j}=R_{j i}$. The degree of coupling $r_{i j}$ results from the relationship between forces and fluxes $[19,20]$ and for diluted and homogeneous solutions is determined by the relations $r_{i j}=-R_{i j}\left(R_{i i} R_{j j}\right)^{-0.5}$ and $r_{i j}=r_{j i}=r$. The second law of thermodynamics imposes the condition $R_{i i} R_{i j} \geq\left(R_{i j}\right)^{2}$, which means that $r_{i j}$ is limited by the relation $-1 \leq r \leq+1$. When $r= \pm 1$, the system is completely coupled, the processes become a single process. When $r=0$, the two processes are completely unrelated and there are no energy conversion interactions. Considering the $r$ factor, Kedem and Caplan presented the expression of the maximum energy conversion efficiency: $e_{\max }=r^{2}\left[1+\left(1-r^{2}\right)^{0.5}\right]^{-2}$ [16]. In turn, Peusner proposed a coupling parameter called "super $Q_{R}$ ": $Q_{R}=r^{2}\left(2-r^{2}\right)^{-1}[15,21]$.

The network form of K-K Equations was presented by Leonardo Peusner [21,22]. He obtained these equations as a result of symmetrical and/or hybrid transformation of classic Kedem-Katchalsky equations with the use of network thermodynamics, which he developed (Peusner NT) [23]. It should be noted that network thermodynamics developed by Oster, Perelson and Katchalsky (Oster, Perelson, Katchalsky NT) also occurs in science [24]. For homogeneous and non-homogeneous binary solutions of nonelectrolytes, there are two symmetrical and two hybrid forms of K-K Equations. Symmetrical forms of these equations contain Peusner matrix coefficients: $R_{i j}$ and $L_{i j}$ (for homogeneous solutions) and $R_{i j}^{r}$ and $L_{i j}^{r}$ (for non-homogeneous solutions), while hybrid forms include Peusner coefficients: $P_{i j}$ and $H_{i j}$ (for homogeneous solutions) and $P_{i j}^{r}$ and $H_{i j}^{r}$ (for non-homogeneous solutions) $(i, j \in\{1,2\})$ [25-28]. It should be noted that solutions which are vigorously mechanically stirred are considered as homogeneous solutions [29,30]. In turn, for heterogeneous solutions (solutions in which concentration polarization occurs), consisting in the formation of concentration boundary layers (CBLs) on both sides of the membrane separating solutions [31-38]. These layers serve as additional kinetic barriers for rapidly penetrating substances through membranes in artificial and biological systems [37-41]. For multicomponent solutions, the number of Peusner matrix coefficients increases: for ternary solutions, there are eight Peusner coefficients: $R_{i j}, L_{i j}, H_{i j}, N_{i j}, K_{i j}, P_{i j}, S_{i j}$ and $W_{i j}$-for homogeneous solutions and $R_{i j}^{r}, L_{i j}^{r}, H_{i j}^{r}, N_{i j}^{r}, K_{i j}^{r}, P_{i j}^{r}, S_{i j}^{r}$ or $W_{i j}^{r}$-for nonhomogeneous solutions $(i, j \in\{1,2,3\}, r=\mathrm{A}$ or B) [42]. It should be noted that the symmetrical forms of these $\mathrm{K}-\mathrm{K}$ Equations, as in the case of binary solutions, include Peusner coefficients $R_{i j}^{r}$ or $L_{i j^{\prime}}^{r}$, while hybrid forms-other Peusner coefficients. It should be noted that the coefficients $R_{i j}^{r}$ or $L_{i j}^{r}$ ' come directly from Onsager thermodynamics, and the remaining coefficients are a consequence of the application of network thermodynamics techniques [25-28,42,43].

In the previous papers $[42,43]$ the case of two directional port of Peusner's network thermodynamics with single inputs for volume flux $J_{v}^{r}$ coupled with thermodynamic force $\Delta P-\Delta \pi_{1}-\Delta \pi_{2}$ and solute fluxes: $J_{1}^{r}$ coupled with thermodynamic force $\Delta \pi_{1} / \bar{C}_{1}$ and $J_{2}^{r}$ coupled with thermodynamic force $\Delta \pi_{2} / \bar{C}_{2}$ was considered. The network K-K Equations for non-homogeneous ternary non-electrolyte solutions containing Peusner's coefficients $H_{i j}^{r}$ and $L_{i j}^{r}(i, j \in\{1,2,3\}, r=\mathrm{A}, \mathrm{B})$ were obtained by means of hybrid network transformations of Peusner's network thermodynamic. The coefficients $H_{i j}^{r}$ and $L_{i j}^{r}(i, j \in\{1,2,3\}$, $r=\mathrm{A}, \mathrm{B})$ occurring in the matrix $\left[H^{r}\right]$ and $\left[L^{r}\right]$ we call Peusner's coefficients and matrix $\left[H^{r}\right]$ or $\left[L^{r}\right]$ —matrix of Peusner's coefficients $H_{i j}^{r}$ or $L_{i j}^{r}$ respectively. According to the principles of network thermodynamic, for non-diagonal coefficients we have $H_{12}^{r} \neq H_{21}^{r}, H_{13}^{r} \neq H_{31}^{r}, H_{23}^{r} \neq H_{32}^{r}, L_{12}^{r} \neq L_{21}^{r}, L_{13}^{r} \neq L_{31}^{r}$ and $L_{23}^{r} \neq L_{32}^{r}$.

The aim of this paper is to develop the form of $R^{r}$ of the K-K Equations, containing the Peusner coefficients $R_{i j}^{r}(i, j \in\{1,2,3\}, r=\mathrm{A}, \mathrm{B})$. We will present the results of calculations of coefficients $R_{i j}^{r}$ and $R_{i j}$ matrix coefficients $R_{\operatorname{det}}^{r}=\operatorname{det}\left[R^{r}\right]$ and $R_{\operatorname{det}}=\operatorname{det}[R]$ and the quotients $\xi_{i j}=\left(R_{i j}^{A}-R_{i j}^{B}\right) / R_{i j}$ and $\xi_{\text {det }}=\left(R_{\text {det }}^{A}-R_{\text {det }}^{B}\right) / R_{\text {det }}$ which were obtained on the basis of experimentally determined coefficients $\left(L_{p}\right.$, $\sigma_{1}, \sigma_{2}, \omega_{11}, \omega_{22}, \omega_{21}, \omega_{12}, \zeta_{1}^{r}$ and $\zeta_{2}^{r}$ ) for glucose in aqueous ethanol solutions and Configurations A and $\mathrm{B}$ of the membrane system. These coefficients were calculated on the basis of experimentally measured volume $\left(J_{v}^{r}\right)$ and solute fluxes $\left(J_{k}^{r}\right)(k=1,2$ and $r=\mathrm{A}, \mathrm{B})$ using the procedure described in [11,30,34]. 
Besides, we will present the results of calculations of the degree of coupling $r_{i j}=-R_{i j}\left(R_{i i} R_{j j}\right)^{-0.5}$ (for homogeneous ternary nonelectrolyte solutions), $r_{i j}^{r}=R_{i j}^{r}\left(R_{i i}^{r} R_{j j}^{r}\right)^{-0.5}$ (for non-homogeneous ternary nonelectrolyte solutions), coupling parameter $Q_{R}=r_{i j} r_{j i}\left(2-r_{i j} r_{j i}\right)^{-1}$ (for homogeneous ternary nonelectrolyte solutions), $Q_{R}^{r}=r_{i j}^{r} r_{j i}^{r}\left(2-r_{i j}^{r} r_{j i}^{r}\right)^{-1}$ (for non-homogeneous ternary nonelectrolyte solutions) and energy conversion coefficients $\left(e_{i j}\right)_{r}=\left(r_{j i}\right)^{2}\left[1+\left(1-r_{i j} r_{j i}\right)^{0.5}\right]^{-2}$ (for homogeneous ternary nonelectrolyte solutions) and $\left(e_{i j}^{r}\right)_{r}=\left(r_{j i}^{r}\right)^{2}\left[1+\left(1-r_{i j}^{r} j_{j i}^{r}\right)^{0.5}\right]^{-2}$ (for non-homogeneous ternary nonelectrolyte solutions) in which $(i, j \in\{1,2,3\}, r=\mathrm{A}, \mathrm{B})$.

\section{Theory}

Similarly, as in previous papers (e.g., [42,43]), let us consider the membrane system presented in Figure 1. In this system the membrane (M) is located in horizontal plane and separates compartments $(l)$ and $(h)$ filled with non-homogeneous ternary non-electrolyte solutions with concentrations at the initial moment $(t=0) C_{k h}$ and $C_{k l}\left(C_{k h}>C_{k l}, k=1,2\right)$. This membrane treated as a "black box" type is isotropic, symmetrical, electroneutral and selective for solvent and non-ionized dissolved substances. For a membrane located in a horizontal plane that is perpendicular to the gravity vector, two configurations of the membrane system are possible. These configurations are denoted by $\mathrm{A}$ and $\mathrm{B}$. In Configuration $\mathrm{A}$, the $C_{k l}$ solution is in the chamber above the membrane, and the $C_{k h}$ solution is in the chamber under the membrane. In Configuration B, the arrangement of the solutions relative to the membrane is reversed.

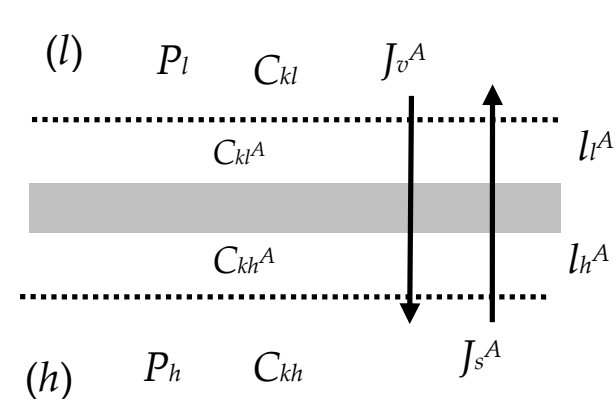

Configuration A

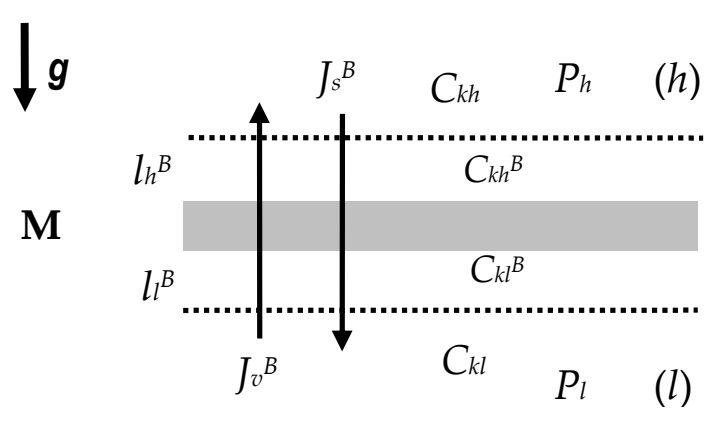

Configuration B

Figure 1. The model of single-membrane system: $\mathrm{M}$-membrane, $g$-gravitational acceleration, $l_{l}^{A}$ and $l_{h}^{A}$ - the concentration boundary layers in Configuration $\mathrm{A}, l_{l}^{B}$ and $l_{h}^{B}$ - the concentration boundary layers in Configuration B, $P_{h}$ and $P_{l}$-mechanical pressures, $C_{k h}$ and $C_{k l}$-global solution concentrations, $C_{k l}^{A}, C_{k h^{\prime}}^{A} C_{k l}^{B}$ and $C_{k h}^{B}$-local (at boundaries between membrane and CBLs) solution concentrations, $J_{k}^{A}$ and $J_{v}^{A}$-solute and volume fluxes in Configuration $A, J_{k}^{B}$ and $J_{v}^{B}$-solute and volume fluxes in Configuration B.

We will consider only isothermal and stationary processes of membrane transport, for which the measure is the volume fluxes $\left(J_{v}^{r}\right)$ and solutes fluxes $\left(J_{k}^{r}\right)(k=1,2$ and $r=\mathrm{A}, \mathrm{B})$. These fluxes can be described by the K-K Equations for ternary non-electrolyte solutions [42,43]. Under such conditions water and solutes which diffuse through the membrane create concentration boundary layers (CBLs), $l_{h}^{r}$ and $l_{l}^{r}$ on both sides of the membrane [35-37]. The thicknesses of $l_{h}^{r}$ and $l_{l}^{r}$ are equal suitably to $\delta_{h}^{r}$ and $\delta_{l}^{r}$. The mean concentrations of solutes "1" and "2" in membrane $\left(\bar{C}_{1}, \bar{C}_{2}\right)$ can be calculated using expressions $\bar{C}_{k}=\left(C_{k h}-C_{k l}\right)\left[\ln \left(C_{k h} C_{k l}{ }^{-1}\right)\right]^{-1}(k=1,2)$. Appearance of CBLs causes that concentrations at the interfaces of the membrane and solutions respectively decreases from $C_{k h}$ to $C_{k h}^{r}$ and increases from $C_{k l}$ to $C_{k l}^{r}\left(C_{k h}^{r}>C_{k l}^{r}, C_{k l}^{r}>C_{k l}, C_{k h}>C_{k h}^{r} . k=1,2\right)$. 
Let us denote by $\rho_{l}^{r}$ and $\rho_{h}^{r}$ the densities of solutions in the interfaces $l_{l}^{r} / \mathrm{M}$ and $\mathrm{M} / l_{h}^{r}$ while by $\rho_{l}$ and $\rho_{h}\left(\rho_{l}<\rho_{h}\right.$ or $\left.\rho_{l}>\rho_{h}\right)$ the density of solutions outside the CBLs. The following conditions can be saved for these densities: $\rho_{l}^{r}>\rho_{l}$ or $\rho_{l}^{r}<\rho_{h^{\prime}}^{r} \rho_{l}^{r}>\rho_{h}^{r}$ or $\rho_{l}^{r}<\rho_{h}^{r}$ and $\rho_{h}^{r}>\rho_{h}$ or $\rho_{h}^{r}<\rho_{h}$. If the solution with lower density is under the membrane, the system $l_{h}^{r} / \mathrm{M} / l_{l}^{r}$ loses its hydrodynamic stability and convective instabilities in near membrane area are observed [35-37]. The measure of the concentration polarization (CP) is the $\mathrm{CP}$ coefficient $\left(\zeta_{k}^{r}\right)$. Using this coefficient, we can write the relation: $C_{k h}^{r}-C_{k l}^{r}=$ $\zeta_{k}^{r}\left(C_{k h}-C_{k l}\right)$. The value of coefficient $\zeta_{k}^{r}$ depends on both the concentration of solutions separated by the membrane $\left(\bar{C}_{k}\right)$ and the configuration of the membrane system $(r=\mathrm{A}, \mathrm{B})$. More specifically for this case, the thicknesses of CBLs $\delta_{h}^{r}$ and $\delta_{l}^{r}$ exceed values $\left(\delta_{h}^{r}\right)_{\text {crit }}$ and $\left(\delta_{l}^{r}\right)_{\text {crit }}$ and CP coefficient $\left(\zeta_{k}^{r}\right)$ exceed its critical value $\left(\zeta_{k}^{r}\right)$ crit suitably $[42,43]$. The dependency between the CP coefficient $\left(\zeta_{k}^{r}\right)$ and the thickness of CBLs $\left(\delta_{h}^{r}\right.$ and $\left.\delta_{l}^{r}\right)$ can be described by the following expression [37].

$$
\zeta_{k}^{r}=\left\{1+R T \omega_{i j}\left[\frac{\delta_{l}^{r}}{\left(D_{i j}^{r}\right)_{l}}+\frac{\delta_{h}^{r}}{\left(D_{i j}^{r}\right)_{h}}\right]\right\}^{-1}
$$

where $(i, j \in\{1,2\}$ and $r=\mathrm{A}, \mathrm{B})$. In diluted non-electrolyte solutions, the diffusion coefficients $\left(D_{k s}^{r}\right)_{l}$ and $\left(D_{k s}^{r}\right)_{h}$ are independent both of gravitational direction and solution concentration. Therefore, we can assume that $\left(D_{k s}^{r}\right)_{l}=\left(D_{k s}^{r}\right)_{h}=D_{k s}$. Besides, we can also assume that $\delta_{h}^{r}=\delta_{l}^{r}=\delta^{r}$.

According to the Kedem-Katchalsky formalism [11] transport properties of the membrane are determined for solutions containing a solvent and two dissolved substances (ternary solution) by practical coefficients: hydraulic permeability $\left(L_{p}\right)$, reflection $\left(\sigma_{k}, k=1,2\right)$ and permeability of solute $\left(\omega_{k f}, k, f \in\{1,2\}\right)$. In turn, the transport properties of the complex $l_{h}^{r} / \mathrm{M} / l_{l}^{r}$ are characterized by coefficients of hydraulic permeability $\left(L_{p}^{r}\right)$, reflection $\left(\sigma_{s k^{\prime}}^{r} \sigma_{a k}^{r}\right)$ and permeability of solute $\left(\omega_{k f}^{r}\right)$. The coefficients of hydraulic, osmotic, advective and diffusive concentration polarization are defined by expressions: $\zeta_{p}^{r}=L_{p}^{r} / L_{p}, \zeta_{v}^{r}=\sigma_{s k}^{r} / \sigma_{k}, \zeta_{a}^{r}=\sigma_{a k}^{r} / \sigma_{k}$ and $\zeta_{k}^{r}=\omega_{k f}^{r} / \omega_{k f}$ [26]. For osmotic volume and diffusive fluxes of homogeneous (evenly stirred) solutions, the values of volume $\left(J_{v}\right)$ and solute $\left(J_{k}\right)$ fluxes does not depend on the configuration of the membrane system. Besides, the dependencies $J_{v}=f\left(C_{k h}-C_{k l}\right)$ and $J_{k}=f\left(C_{k h}-C_{k l}\right)$ are linear, while $J_{v}^{r}=f\left(C_{k h}-C_{k l}\right)$ and $J_{k}^{r}=f\left(C_{k h}-C_{k l}\right)$ are nonlinear $[33,43]$. The formation of the layers $l_{l}^{r}$ and $l_{h}^{r}$ reduce the value of volume and solute fluxes from $J_{v}$ and $J_{k}$ (in conditions of homogeneous solutions) to $J_{v}^{r}$ and $J_{k}^{r}$ (in condition of $\mathrm{CP}$ ), respectively.

The Kedem-Katchalsky Equations for $\mathrm{CP}$ conditions can be written as:

$$
\begin{gathered}
J_{v}^{r}=\zeta_{p}^{r} L_{p}\left(\Delta P-\zeta_{v 1}^{r} \sigma_{1} \Delta \pi_{1}-\zeta_{v 2}^{r} \sigma_{2} \Delta \pi_{2}\right) \\
J_{1}^{r}=\zeta_{s 11}^{r} \omega_{11} \Delta \pi_{1}+\zeta_{s 12}^{r} \omega_{12} \Delta \pi_{2}+\bar{C}_{1}\left(1-\zeta_{a 1}^{r} \sigma_{1}\right) J_{v}^{r} \\
J_{2}^{r}=\zeta_{s 21}^{r} \omega_{21} \Delta \pi_{1}+\zeta_{s 22}^{r} \omega_{22} \Delta \pi_{2}+\bar{C}_{1}\left(1-\zeta_{a 2}^{r} \sigma_{2}\right) J_{v}^{r}
\end{gathered}
$$

where $J_{v}^{r}, J_{1}^{r}$ and $J_{2}^{r}$-volume and solutes, $1 "$ and, $2 "$ fluxes respectively, $L_{p}$-hydraulic permeability coefficient, $\sigma_{1}$ and $\sigma_{2}$-reflection coefficients suitably for solutes "1" or ",2", $\omega_{11}$ and $\omega_{22}$-solute permeability coefficients for solutes " 1 " or " 2 " generated by forces with indexes " 1 " or ",2" and $\omega_{12}$ and $\omega_{21}$ - cross coefficients of permeability for substances " 1 " or " 2 " generated by forces with indexes , 2 " or "1" respectively. $\Delta P=P_{h}-P_{l}$ is the hydrostatic pressure difference $\left(P_{h}, P_{l}\right.$ are higher and lower values of hydrostatic pressure suitably). $\Delta \pi_{k}=R T\left(C_{k h}-C_{k l}\right)$ is the difference of osmotic pressure ( $R T$ is the product of gas constant and thermodynamic temperature whereas $C_{k h}$ and $C_{k l}$ are solutes concentrations, $\left.k=1,2\right)$. $\bar{C}_{k}$ is the mean solute concentration in membrane and is expressed by $\bar{C}_{k}=\left(C_{k h}-C_{k l}\right)\left[\ln \left(C_{k h} C_{k l}{ }^{-1}\right)\right]^{-1}$ $(k=1,2)$. By means of this expression one can show that $\Delta \pi_{k} / \bar{C}_{k}=\ln \left(C_{k h} C_{k l}^{-1}\right)$. Equations (2)-(4) are modified Kedem-Katchalsky Equations for ternary solutions [33]. 
The Equations (2)-(4) can be transformed by simple algebraic transformations to the matrix form of the Kedem-Katchalsky-Peusner equations for non-homogenous non-electrolyte ternary solutions:

$$
\left[\begin{array}{c}
\Delta P-\Delta \pi_{1}-\Delta \pi_{2} \\
\frac{\Delta \pi_{1}}{\overline{\bar{C}}_{1}} \\
\frac{\Delta \pi_{2}}{\overline{\mathrm{C}}_{2}}
\end{array}\right]=\left[\begin{array}{lll}
R_{11}^{r} & R_{12}^{r} & R_{13}^{r} \\
R_{21}^{r} & R_{22}^{r} & R_{23}^{r} \\
R_{31}^{r} & R_{32}^{r} & R_{33}^{r}
\end{array}\right]\left[\begin{array}{c}
J_{v}^{r} \\
J_{1}^{r} \\
J_{2}^{r}
\end{array}\right]=\left[R^{r}\right]\left[\begin{array}{c}
J_{v}^{r} \\
J_{1}^{r} \\
J_{2}^{r}
\end{array}\right]
$$

where $R_{11}^{r}=\left(\zeta_{p}^{r} L_{p}\right)^{-1}-\left[\left(1-\zeta_{v 1}^{r} \sigma_{1}\right)\left(\alpha_{1}-\alpha_{2}\right)+\left(1-\zeta_{v 2}^{r} \sigma_{2}\right)\left(\alpha_{3}-\alpha_{4}\right)\right] \gamma^{-1}, \alpha_{1}=\zeta_{s 12}^{r} \omega_{12}\left(1-\zeta_{a 2}^{r} \sigma_{2}\right) \bar{C}_{2}$, $\alpha_{2}=\zeta_{s 22}^{r} \omega_{22}\left(1-\zeta_{a 1}^{r} \sigma_{1}\right) \bar{C}_{1}, \quad \alpha_{3}=\zeta_{s 21}^{r} \omega_{21}\left(1-\zeta_{a 1}^{r} \sigma_{1}\right) \bar{C}_{1}, \quad \alpha_{4}=\zeta_{s 11}^{r} \omega_{11}\left(1-\zeta_{a 2}^{r} \sigma_{2}\right) \bar{C}_{2}, \quad \gamma=$ $\zeta_{s 11}^{r} \omega_{11} \zeta_{s 22}^{r} \omega_{22}-\zeta_{s 12}^{r} \omega_{12} \zeta_{s 21}^{r} \omega_{21}, \quad R_{12}^{r}=\left[\zeta_{s 21}^{r} \omega_{21}\left(1-\zeta_{v 2}^{r} \sigma_{2}\right)-\zeta_{s 22}^{r} \omega_{22}\left(1-\zeta_{v 1}^{r} \sigma_{1}\right)\right] \gamma^{-1}, \quad R_{13}^{r}=$ $\left[\zeta_{s 12}^{r} \omega_{12}\left(1-\zeta_{v 1}^{r} \sigma_{1}\right)-\zeta_{s 11}^{r} \omega_{11}\left(1-\zeta_{v 2}^{r} \sigma_{2}\right)\right] \gamma^{-1}, R_{21}^{r}=\left[\zeta_{s 12}^{r} \omega_{12}\left(1-\zeta_{a 2}^{r} \sigma_{2}\right) \bar{C}_{2}-\zeta_{s 22}^{r} \omega_{22}\left(1-\zeta_{a 1}^{r} \sigma_{1}\right) \bar{C}_{1}\right] \gamma^{-1}$, $R_{22}^{r}=\zeta_{s 22}^{r} \omega_{22} \gamma^{-1} \bar{C}_{1}^{-1} R_{23}^{r}=-\zeta_{s 12}^{r} \omega_{12} \gamma^{-1} \bar{C}_{1}^{-1}, R_{31}^{r}=\left[\zeta_{s 21}^{r}\left(1-\zeta_{a 1}^{r} \sigma_{1}\right) \bar{C}_{1}-\zeta_{s 11}^{r} \omega_{11}\left(1-\zeta_{a 2}^{r} \sigma_{2}\right) \bar{C}_{2}\right] \gamma^{-1}$, $R_{32}^{r}=-\zeta_{s 21}^{r} \omega_{21} \gamma^{-1} \bar{C}_{2}^{-1}, R_{33}^{r}=\zeta_{s 11}^{r} \omega_{11} \gamma^{-1} \bar{C}_{2}{ }^{-1},\left[R^{r}\right]$ is the matrix of the Peusner's coefficients $R_{i j}^{r}(\mathrm{i}, \mathrm{j} \in\{1,2,3\})$ for ternary non-electrolyte solutions in conditions of concentration polarization.

Results from Equation (5) are the non-diagonal coefficients $R_{12}^{r} \neq R_{21}^{r}, R_{13}^{r} \neq R_{31}^{r}$ and $R_{23}^{r} \neq R_{32}^{r}$. Besides, the determinant of the matrix $\left[R^{r}\right]$ is equal to:

$$
\operatorname{det}\left[R^{r}\right]=\frac{1}{\zeta_{p}^{r} L_{p} \bar{C}_{1} \bar{C}_{2}\left(\omega_{11} \zeta_{s 11}^{r} \omega_{22} \zeta_{s 22}^{r}-\omega_{12} \zeta_{s 12}^{r} \omega_{21} \zeta_{s 21}^{r}\right)} \equiv R_{d e t}^{r}
$$

Index ", $r^{\prime \prime}$ in Equations (2)-(6) indicate that the fluxes $J_{v}^{r}, J_{1}^{r}, J_{2}^{r}$, Coefficients $R_{i j}^{r}(i, j \in\{1,2,3\}$ and matrix $\left[R^{r}\right]$ of these coefficients ( $R^{r}$ form of the matrix of Peusner's coefficients), depend on configuration of the membrane system $(r=\mathrm{A}, \mathrm{B})$. From a formal point of view, the case of $R_{\text {det }}^{r}=0$ is excluded, because in order for the denominator of Equation (6) to be different from zero, the condition $\omega_{11} \zeta_{s 11}^{r} \omega_{22} \zeta_{s 22}^{r} \neq \omega_{12} \zeta_{s 12}^{r} \omega_{21} \zeta_{s 21}^{r}$ must be satisfied. If $\omega_{11} \zeta_{s 11}^{r} \omega_{22} \zeta_{s 22}^{r}>\omega_{12} \zeta_{s 12}^{r} \omega_{21} \zeta_{s 21}^{r}$ then $R_{d e t}^{r}>0$, and if $\omega_{11} \zeta_{s 11}^{r} \omega_{22} \zeta_{s 22}^{r}<\omega_{12} \zeta_{s 12}^{r} \omega_{21} \zeta_{s 21}^{r}$ then $R_{\text {det }}^{r}<0$.

In order to write Equations (5) and (6) for the conditions of homogeneity of solutions, the superscript " $\mathrm{r}$ " should be removed and assumption that the condition $\zeta_{p}^{r}=\zeta_{v 1}^{r}=\zeta_{v 2}^{r}=\zeta_{a 1}^{r}=\zeta_{a 2}^{r}=\zeta_{s 11}^{r}$ $=\zeta_{s 12}^{r}=\zeta_{s 22}^{r}=\zeta_{s 21}^{r}=1$ is fulfilled. Then Equations (5) and (6) are taking the following form:

$$
\left[\begin{array}{c}
\Delta P-\Delta \pi_{1}-\Delta \pi_{2} \\
\frac{\Delta \pi_{1}}{\bar{C}_{1}} \\
\frac{\Delta \pi_{2}}{\bar{C}_{2}}
\end{array}\right]=\left[\begin{array}{lll}
R_{11} & R_{12} & R_{13} \\
R_{21} & R_{22} & R_{23} \\
R_{31} & R_{32} & R_{33}
\end{array}\right]\left[\begin{array}{c}
J_{v} \\
J_{1} \\
J_{2}
\end{array}\right]=[R]\left[\begin{array}{c}
J_{v} \\
J_{1} \\
J_{2}
\end{array}\right]
$$

where $R_{11}^{r}=\left(\zeta_{p}^{r} L_{p}\right)^{-1}-\left[\left(1-\zeta_{v 1}^{r} \sigma_{1}\right)\left(\alpha_{1}-\alpha_{2}\right)+\left(1-\zeta_{v 2}^{r} \sigma_{2}\right)\left(\alpha_{3}-\alpha_{4}\right)\right] \gamma^{-1}, \alpha_{1}=\omega_{12}\left(1-\sigma_{2}\right) \bar{C}_{2}$, $\alpha_{2}=\omega_{22}\left(1-\sigma_{1}\right) \bar{C}_{1}, \quad \alpha_{3}=\omega_{21}\left(1-\sigma_{1}\right) \bar{C}_{1}, \quad \alpha_{4}=\omega_{11}\left(1-\sigma_{2}\right) \bar{C}_{2}, \quad \gamma=\omega_{11} \omega_{22}-$ $\omega_{12} \omega_{21}, \quad R_{12}=\left[\omega_{21}\left(1-\sigma_{2}\right)-\omega_{22}\left(1-\sigma_{1}\right)\right] \gamma^{-1}, \quad R_{13}=\left[\omega_{12}\left(1-\sigma_{1}\right)-\omega_{11}\left(1-\sigma_{2}\right)\right] \gamma^{-1}, R_{21}=$ $\left[\omega_{12}\left(1-\sigma_{2}\right) \bar{C}_{2}-\omega_{22}\left(1-\sigma_{1}\right) \bar{C}_{1}\right] \gamma^{-1}, \quad R_{22}=\omega_{22} \gamma^{-1} \bar{C}_{1}^{-1}, \quad R_{23}=-\omega_{12} \gamma^{-1} \bar{C}_{1}^{-1}, \quad R_{31}=$ $\left[\omega_{21}\left(1-\sigma_{1}\right) \bar{C}_{1}-\omega_{11}\left(1-\sigma_{2}\right) \bar{C}_{2}\right] \gamma^{-1}, R_{32}=-\omega_{21} \gamma^{-1} \bar{C}_{2}^{-1}, R_{33}=\omega_{11} \gamma^{-1} \bar{C}_{2}^{-1}$.

Besides the determinant of matrix $[R]$ is given by the relationship:

$$
\operatorname{det}[R]=\frac{1}{L_{p} \bar{C}_{1} \bar{C}_{2}\left(\omega_{11} \omega_{22}-\omega_{12} \omega_{21}\right)} R_{\operatorname{det}}
$$

As in the case of Equation (6), the case of $R_{\text {det }}=0$ is excluded, because in order for the denominator of Equation (8) to be different from zero, the condition $\omega_{11} \omega_{22} \neq \omega_{12} \omega_{21}$ must be fulfilled. If $\omega_{11} \omega_{22}>\omega_{12} \omega_{21}$ then $R_{\text {det }}^{r}>0$, and if $\omega_{11} \omega_{22}<\omega_{12} \omega_{21}$ then $R_{\text {det }}<0$. 
The coefficients $R_{11}, R_{12}, R_{13}, R_{21}, R_{22}, R_{23}, R_{31}, R_{32}$ and $R_{33}$ occurring in the matrix [R] we call Peusner's coefficients and matrix $[R]-R$ form of the matrix of Peusner's coefficients. According to the principles of network thermodynamic [15] in the above equation, symmetry of non-diagonal coefficients $\left(R_{i j}=R_{j i} i \neq j\right)$ is not required. In the case considered above for non-diagonal coefficients, we have $R_{12}=R_{21}, R_{13}=R_{31}$ only when $\omega_{12}=\omega_{21}$. Besides, from Equation (7) it results that $R_{23}=R_{32}$ only when $\omega_{12} \bar{C}_{1}=\omega_{21} \bar{C}_{2}$.

In order to show the relations between coefficients $R_{i j}^{r}$ and $R_{i j}$ and between determinants of matrixes $\left[R^{r}\right]$ and $[R]$ for $\mathrm{A}$ and $\mathrm{B}$ configurations of the membrane system $(r=\mathrm{A}, \mathrm{B})$ we calculate using Equations (4)-(7) the expressions:

$$
\begin{gathered}
\xi_{i j}=\frac{R_{i j}^{A}-R_{i j}^{B}}{R_{i j}} \\
\xi_{\text {det }}=\frac{R_{\text {det }}^{A}-R_{\text {det }}^{B}}{R_{\text {det }}}
\end{gathered}
$$

The values of coefficients $\xi_{i j}$ and $\xi_{\text {det }}$ show the influence of CP and natural convection (NC) on the membrane transport. These coefficients are a measure of the distance of convective processes from the critical state (non-convection). Assuming that the coefficients $R_{i j}^{A}, R_{i j^{\prime}}^{B} R_{i j}, R_{d e t}^{A} R_{d e t^{\prime}}^{B} \xi_{i j}$ and $\xi_{d e t}$ have the same sign, on the basis of Equations (9) and (10), we can write the criteria listed in Table 1.

\begin{tabular}{|c|c|c|c|}
\hline$R_{i j}^{A}>0, R_{i j}^{B}>0, R_{i j}>0$ & $\begin{array}{l}R_{i j}^{A}>R_{i j}, R_{i j}^{B}>R_{i j} \\
R_{i j}^{A}<R_{i j}, R_{i j}^{B}<R_{i j}\end{array}$ & $\begin{array}{l}R_{i j}^{A}>R_{i j}^{B} \\
R_{i j}^{A}<R_{i j}^{B} \\
R_{i j}^{A}=R_{i j}^{B}\end{array}$ & $\begin{array}{l}\xi_{i j}>0 \\
\xi_{i j}<0 \\
\xi_{i j}=0\end{array}$ \\
\hline$R_{i j}^{A}<0, R_{i j}^{B}<0, R_{i j}<0$ & $\begin{array}{l}R_{i j}^{A}>R_{i j}, R_{i j}^{B}>R_{i j} \\
R_{i j}^{A}<R_{i j}, R_{i j}^{B}<R_{i j}\end{array}$ & $\begin{array}{l}R_{i j}^{A}>R_{i j}^{B} \\
R_{i j}^{A}<R_{i j}^{B} \\
R_{i j}^{A}=R_{i j}^{B}\end{array}$ & $\begin{array}{l}\xi_{i j}<0 \\
\xi_{i j}>0 \\
\xi_{i j}=0\end{array}$ \\
\hline$R_{d e t}^{A}>0, R_{d e t}^{B}>0, R_{d e t}>0$ & $\begin{array}{l}R_{d e t}^{A}>R_{d e t}, R_{d e t}^{B}>R_{d e t} \\
R_{d e t}^{A}<R_{d e t}, R_{d e t}^{B}<R_{d e t}\end{array}$ & $\begin{array}{l}R_{\text {det }}^{A}>R_{\text {det }}^{B} \\
R_{\text {det }}^{A}<R_{\text {det }}^{B} \\
R_{\text {det }}^{A}=R_{\text {det }}^{B}\end{array}$ & $\begin{array}{l}\xi_{\text {det }}>0 \\
\xi_{\text {det }}<0 \\
\xi_{\text {det }}=0\end{array}$ \\
\hline$R_{d e t}^{A}<0, R_{d e t}^{B}<0, R_{d e t}<0$ & $\begin{array}{l}R_{d e t}^{A}>R_{d e t}, R_{d e t}^{B}>R_{d e t} \\
R_{d e t}^{A}<R_{d e t}, R_{d e t}^{B}<R_{d e t}\end{array}$ & $\begin{array}{l}R_{\text {det }}^{A}>R_{\text {det }}^{B} \\
R_{\text {det }}^{A}<R_{\text {det }}^{B} \\
R_{\text {det }}^{A}=R_{\text {det }}^{B}\end{array}$ & $\begin{array}{l}\xi_{\text {det }}<0 \\
\xi_{\text {det }}>0 \\
\xi_{\text {det }}=0\end{array}$ \\
\hline
\end{tabular}

Table 1. Criteria for coefficients, $R_{i j}^{A}, R_{i j^{\prime}}^{B}, R_{i j}, R_{d e t^{\prime}}^{A}, R_{d e t^{\prime}}^{B} \xi_{i j}$ and $\xi_{d e t}$.

In order to show the relationship between coefficients $R_{i j}, R_{j i}, R_{i i}$ and $R_{j j}$ and coefficients $R_{i j}^{r}, R_{j i}^{r}, R_{i i}^{r}$ and $R_{j j}^{r}$ for $\mathrm{A}$ and $\mathrm{B}$ configurations of membrane system we will calculate the Kedem-Caplan-Peusner (KCP) degree of coupling $r_{i j}$ and $r_{i j}^{r}$ in which $i, j \in\{1,2,3\}$, superscript $r=\mathrm{A}, \mathrm{B}$, using Equations (5), (7), (11) and (12) $[19,20]$. The expressions for these coefficients take the following forms:

$$
\begin{gathered}
r_{i j}^{r}=-\frac{R_{i j}^{r}}{\sqrt{R_{i i}^{r} R_{j j}^{r}}} \\
r_{i j}=-\frac{R_{i j}}{\sqrt{R_{i i} R_{j j}}}
\end{gathered}
$$

The second law of thermodynamics imposes the conditions $R_{i i}^{r} R_{j j}^{r} \geq\left(R_{i j}^{r}\right)^{2}$ and $R_{i i}^{r} R_{j j}^{r} \geq\left(R_{j i}^{r}\right)^{2}$ which means that $r_{i j}^{r}$ and $r_{j i}^{r}$ is limited by the relation $-1 \leq r_{i j}^{r} r_{j i}^{r} \leq+1$. For ternary solutions, taking into 
consideration Equations (5) and (11) and (7) and (12) we get: $r_{12}^{r} \neq r_{21}^{r}, r_{13}^{r} \neq r_{31}^{r}, r_{23} \neq r_{32}$ and $r_{23}^{r} \neq r_{32}^{r}$. This shows that for conditions of $\mathrm{CP}$, Onsager's reciprocal relations are not satisfied.

The $\left(e_{i j}\right)_{r}$ and $\left(e_{i j}^{r}\right)_{r}$ coefficients can be used to evaluate of energy conversion efficiency by means of the Kedem-Caplan-Peusner coefficient, which can be written in the form:

$$
\begin{aligned}
\left(e_{i j}^{r}\right)_{r} & =\frac{\left(r_{j i}^{r}\right)^{2}}{\left(1+\sqrt{1-r_{i j}^{r} j_{j i}^{r}}\right)^{2}}=\frac{\left(R_{j i}^{r}\right)^{2}}{R_{i i}^{r} R_{j j}^{r}\left(1+R_{i i}^{r} R_{j j}^{r} \sqrt{R_{i i}^{r} R_{j j}^{r}-R_{i j}^{r} R_{j i}^{r}}\right)^{2}} \\
\left(e_{i j}\right)_{r} & =\frac{\left(r_{j i}\right)^{2}}{\left(1+\sqrt{1-r_{i j} r_{j i}}\right)^{2}}=\frac{\left(R_{j i}\right)^{2}}{R_{i i} R_{j j}\left(1+R_{i i} R_{j j} \sqrt{R_{i i} R_{j j}-R_{i j} R_{j i}}\right)^{2}}
\end{aligned}
$$

Peusner proposed the "super $Q_{R}$ "—coupling parameter, defined by the following expression [15,21,22]:

$$
\begin{aligned}
& Q_{R}^{r}=\frac{R_{i j}^{r} R_{j i}^{r}}{2 R_{i i}^{r} R_{j j}^{r}-R_{i j}^{r} R_{j i}^{r}}=\frac{r_{i j}^{r} r_{j i}^{r}}{2-r_{i j}^{r} r_{j i}^{r}} \\
& Q_{R}=\frac{R_{i j} R_{j i}}{2 R_{i i} R_{j j}-R_{i j} R_{j i}}=\frac{r_{i j} r_{j i}}{2-r_{i j} r_{j i}}
\end{aligned}
$$

\section{Results and Discussion}

For ternary solutions, the coefficients $R_{i j}^{r} R_{i j},(i, j \in\{1,2,3\}, r=\mathrm{A}, \mathrm{B})$ and determinant of matrix of these coefficients det $\left[R^{r}\right]$ were calculated for polymer membrane Nephrophan (VEB Filmfabrik, Wolfen, Germany) and glucose solutions in aqueous solution of ethanol using Equations (2)-(16). Nephrophan is a microporous, highly hydrophilic membrane made of cellulose acetate (cello- triacetate $\left.\left(\mathrm{OCO}-\mathrm{CH}_{3}\right)_{n}\right)$. The glucose concentration was marked by Index " 1 " and the ethanol concentration by Index " 2 ". The concentration of Substance " 1 " in Chamber (h) take values from $C_{1 h}=1 \mathrm{~mol} / \mathrm{m}^{3}$ to $C_{1 h}=101 \mathrm{~mol} / \mathrm{m}^{3}$. In turn, concentration of a Substance "2" in Chamber (h) was constant and amounted to $C_{2 h}=201 \mathrm{~mol} / \mathrm{m}^{3}$. The concentrations of both components in the chamber (l) were established and amounted to $C_{1 l}=C_{2 l}=1 \mathrm{~mol} \mathrm{~m}^{-3}$. In expressions under Equation (2) which describe the matrix coefficients $R_{11}^{r}, R_{12}^{r}, R_{13}^{r}, R_{21}^{r}, R_{22}^{r}, R_{23}^{r}, R_{31}^{r}, R_{32}^{r}$ and $R_{33}^{r}$ which are the coefficients that describe transport properties of membrane $\left(L_{p}, \sigma_{1}, \sigma_{2}, \omega_{11}, \omega_{22}, \omega_{21}\right.$ and $\left.\omega_{12}\right)$, average concentrations of Solutions " 1 " and " 2 " in the membrane $\left(\bar{C}_{1}, \bar{C}_{2}\right)$ and CP coefficients $\left(\zeta_{p}^{r}, \zeta_{a 1}^{r}, \zeta_{a 2}^{r}, \zeta_{v 1}^{r}, \zeta_{s 11}^{r}, \zeta_{s 12}^{r}, \zeta_{v 2}^{r}, \zeta_{s 22}^{r}\right.$ and $\left.\zeta_{s 21}^{r}\right)$. For Nephrophan membrane and aqueous solutions of glucose and ethanol the following conditions are fulfilled: $\zeta_{p}^{r}=\zeta_{a 1}^{r}=\zeta_{a 2}^{r}=1, \zeta_{v 1}^{r}=\zeta_{s 11}^{r}=\zeta_{s 12}^{r}=\zeta_{1}^{r}$ and $\zeta_{v 2}^{r}=\zeta_{s 22}^{r}=\zeta_{s 21}^{r}=\zeta_{2}^{r}$ [42]. The coefficients describing transport properties of membrane, e.g., hydraulic permeability $\left(L_{p}\right)$, reflection $\left(\sigma_{1}, \sigma_{2}\right)$ and diffusive permeability $\left(\omega_{11}, \omega_{22}, \omega_{21}, \omega_{12}\right)$ were appointed in the conditions of uniform stirring of solutions separated by membrane in series of independent experiments according with the procedure described in the paper [11]. For Nephrophan, membrane values of these coefficients are independent on solution concentration and amount to $L_{p}=4.9 \times 10^{-12} \mathrm{~m}^{3} / \mathrm{Ns}, \sigma_{1}=0.068, \sigma_{2}=0.025, \omega_{11}=0.8 \times 10^{-9} \mathrm{~mol} / \mathrm{Ns}$, $\omega_{12}=0.81 \times 10^{-13} \mathrm{~mol} / \mathrm{Ns}, \omega_{22}=1.43 \times 10^{-9} \mathrm{~mol} / \mathrm{Ns}$ and $\omega_{21}=1.63 \times 10^{-12} \mathrm{~mol} / \mathrm{Ns}[33]$.

\subsection{Concentration Dependencies of Coefficients $\zeta_{i}^{r}$ and $\rho^{r}$}

In Figure 2, the experimental dependencies $\zeta_{i}^{r}=f\left(\bar{C}_{1}, \bar{C}_{2}=37.71 \mathrm{~mol} / \mathrm{m}^{3}\right),(i=1$ or 2 and $r=\mathrm{A}$ or B) were presented for glucose solutions in $201 \mathrm{~mol} \mathrm{~m}^{-3}$ aqueous solution of ethanol taken from our previous paper [42]. The dependences $\rho^{r}=f\left(\bar{C}_{1}, \bar{C}_{2}=37.71 \mathrm{~mol} / \mathrm{m}^{3}\right),(i=1$ or 2 and $r=\mathrm{A}$ or B) presented in Figure 3 were calculated on the basis of Equation (1) and the results shown in 
Figure 2. The points $(\bigcirc, \triangle)$ were obtained for Configuration A and points $(\square, \nabla)$ for Configuration B of single-membrane system.

Figure 2 shows that in the case of Configuration A for $0<\bar{C}_{1} \leq 4 \mathrm{~mol} / \mathrm{m}^{3}, \zeta_{1}^{A}=\zeta_{2}^{A}=0.5=$ constant and for $4 \mathrm{~mol} / \mathrm{m}^{3}<\bar{C}_{1} \leq 12.72 \mathrm{~mol} / \mathrm{m}^{3}$ the values of coefficients $\zeta_{1}^{A}$ and $\zeta_{2}^{A}$ decrease nonlinearly and for $\bar{C}_{1}>12.72 \mathrm{~mol} / \mathrm{m}^{3}$ reach constant value equal respectively to $\zeta_{1}^{A}=\zeta_{2}^{A}=0.03$. In the case of Configuration B for $0<\bar{C}_{1} \leq 5.41 \mathrm{~mol} / \mathrm{m}^{3}, \zeta_{1}^{B}=\zeta_{2}^{B}=0.03=$ constant, and for $5.41 \mathrm{~mol} / \mathrm{m}^{3}<\bar{C}_{1} \leq 12.72$ $\mathrm{mol} / \mathrm{m}^{3}$ the values of coefficients $\zeta_{1}^{A}$ and $\zeta_{2}^{A}$ increase and for $\bar{C}_{1}>12.72 \mathrm{~mol} / \mathrm{m}^{3}$ reach constant value equal respectively to $\zeta_{1}^{A}=\zeta_{2}^{A}=0.5$. The results presented in this figure show that $0.5 \geq \zeta_{1}^{A} \geq 0.03$ and $0.03 \leq \zeta_{1}^{B} \leq 0.5$. This notation indicates that for the same values $\bar{C}_{1}$ and $\bar{C}_{2}$ the value of coefficient $\zeta_{1}^{A}$ decreases from 0.5 to 0.03 and coefficient $\zeta_{1}^{B}$ increases from 0.03 to 0.5 .

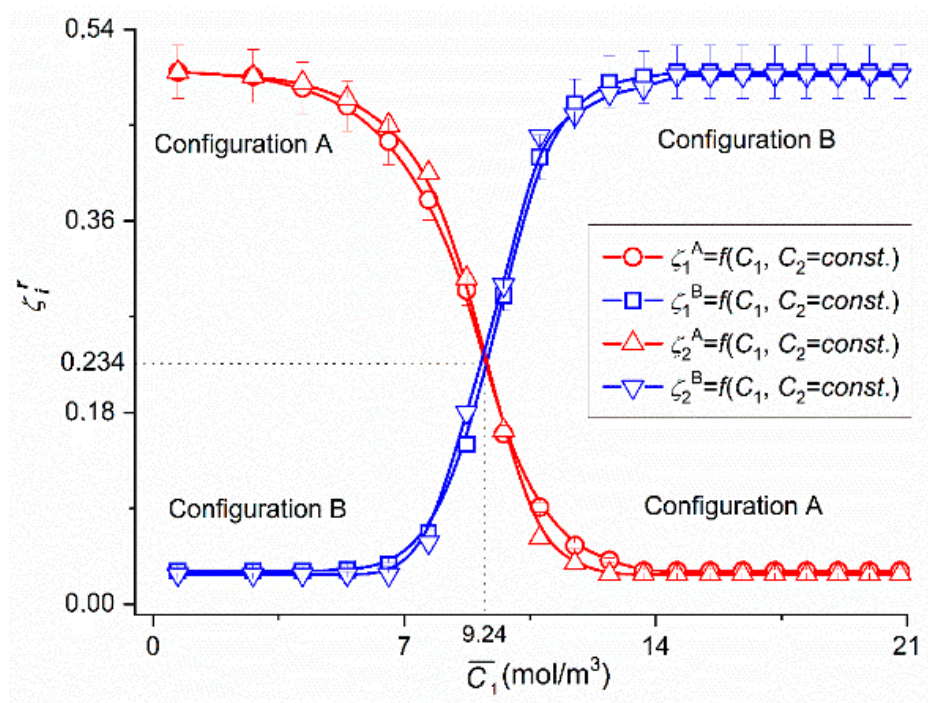

Figure 2. Dependencies of concentration polarization coefficient $\left(\zeta_{i}^{r}\right)$ on glucose concentration in $201 \mathrm{~mol} / \mathrm{m}^{3}$ aqueous ethanol solution for Configurations A and B of the single-membrane system.

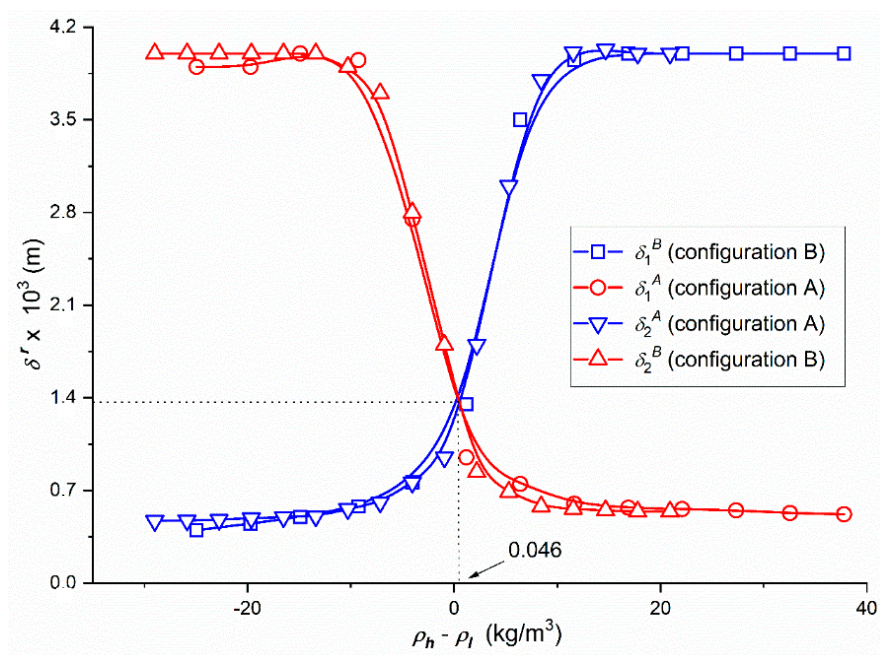

Figure 3. Dependencies of the thickness of concentration boundary layers $\left(\delta^{r}\right)$ in Configurations $\mathrm{A}(r=A)$ and $\mathrm{B}(r=B)$ of the membrane system on density difference $\left(\rho_{h}-\rho_{l}\right)$ of glucose concentration in $201 \mathrm{~mol} / \mathrm{m}^{3}$ aqueous ethanol solutions.

Figure 3 shows that in Configuration A for $-30 \mathrm{~kg} / \mathrm{m}^{-3}<\Delta \rho=\rho_{h}-\rho_{l} \leq-8.5 \mathrm{~kg} / \mathrm{m}^{-3}$, $\delta^{A}=3.95 \times 10^{-3} \mathrm{~m}=$ constant and for $-8.5 \mathrm{~kg} / \mathrm{m}^{-3}<\Delta \rho \leq 7.9 \mathrm{~kg} / \mathrm{m}^{-3}$ the values of coefficients $\delta^{A}$ decrease nonlinearly and for $\Delta \rho>7.9 \mathrm{~kg} / \mathrm{m}^{-3}$ reach constant value equal respectively to $\delta^{A}=0.52 \times 10^{-3} \mathrm{~m}$ 
$=$ constant. For $-30 \mathrm{~kg} / \mathrm{m}^{-3}<\Delta \rho \leq-8.5 \mathrm{~kg} / \mathrm{m}^{-3}, \delta^{B}=0.52 \times 10^{-3} \mathrm{~m}=$ constant and for $-8.5 \mathrm{~kg} / \mathrm{m}^{-3}$ $<\Delta \rho \leq 7.9 \mathrm{~kg} / \mathrm{m}^{-3}$ the values of coefficients $\delta^{B}$ decrease nonlinearly and for $\Delta \rho>7.9 \mathrm{~kg} / \mathrm{m}^{-3}$ reach constant value equal respectively to $\delta^{B}=3.95 \times 10^{-3} \mathrm{~m}=$ const. The results presented in Figure 3 show that $3.95 \times 10^{-3} \mathrm{~m} \geq \delta^{A} \geq 0.52 \times 10^{-3} \mathrm{~m}$ and $0.52 \times 10^{-3} \mathrm{~m} \leq \delta^{B} \leq 3.95 \times 10^{-3} \mathrm{~m}$. This notation indicates that for the same values $\Delta \rho$ the value of coefficient $\delta^{A}$ decreases from $3.95 \times 10^{-3} \mathrm{~m}$ to $0.52 \times 10^{-3} \mathrm{~m}$ and coefficient $\delta^{B}$ increases from $0.52 \times 10^{-3} \mathrm{~m}$ to $3.95 \times 10^{-3} \mathrm{~m}$.

In addition, it can be seen from the Figures 2 and 3 that for $\bar{C}_{1}<9.24 \mathrm{~mol} / \mathrm{m}^{3}$ and $\Delta \rho \leq 0.046 \mathrm{~kg} / \mathrm{m}^{-3}$ in Configuration A, the complex of CBLs is hydrodynamically unstable and in Configuration B-hydrodynamically stable, because the solutions of ethanol prevailing over glucose are under the membrane, and for that case the solution density under the membrane is lower than the solution density over the membrane. In Configuration B, the complex of CBLs is stable because density of the solution under the membrane is greater than the solution above the membrane. In turn for $\bar{C}_{1}>9.24 \mathrm{~mol} / \mathrm{m}^{3}$ and $\Delta \rho>0.046 \mathrm{~kg} / \mathrm{m}^{-3}$ in Configuration A, the complex of CBLs is hydrodynamically stable, and in Configuration B-hydrodynamically unstable due to the fact that in solutions separated by the membrane, glucose concentration is greater than ethanol and density of solution under the membrane is greater than the solution over the membrane. In Configuration B, the complex of CBLs is unstable because density of the solution under the membrane is smaller than the solution above the membrane. This causes the convection movements vertically downward. For $\bar{C}_{1}=9.24 \mathrm{~mol} / \mathrm{m}^{3}$ and $\Delta \rho=0.046 \mathrm{~kg} / \mathrm{m}^{-3}$ the CBLS complex is independent of the membrane system configuration and therefore $\zeta_{1}^{A}=\zeta_{1}^{B}=0.234$ and $\delta^{A}=\delta^{B}=1.3 \times 10^{-3} \mathrm{~m}$. In Configuration A, a non-convective state occurs, when the density of the solution in the compartment above the membrane is higher than density of the solution in the compartment under the membrane. In Configuration A natural convection occurs when $\rho_{l}>\rho_{e}^{A}, \rho_{i}^{A}>\rho_{h}$ and $\rho_{e}^{A}>\rho_{i}^{A}$ and is directed vertically upwards. On the other hand, in Configuration B, a natural convection occurs when $\rho_{l}<\rho_{e}^{B}, \rho_{i}^{B}<\rho_{h}$ and $\rho_{e}^{B}<\rho_{e i}^{B}$ and is directed vertically downwards [38]. Natural convection allows it to increase the value fluxes of $J_{v k}^{r}$ and $J_{k}^{r}$.

\subsection{Concentration Dependencies of Coefficients $R_{i j^{\prime}}^{r}, R_{i j}, R_{\text {det }}^{r}$ and $R_{\text {det }}$}

To calculate $R_{i j}^{r}, R_{i j}, R_{d e t}^{r}$ and $R_{d e t},(i, j \in\{1,2,3\}, r=\mathrm{A}, \mathrm{B})$, based on Equations (5)-(8) respectively, the characteristics $\zeta_{1}^{r}=f\left(\bar{C}_{1}, \bar{C}_{2}=37.71 \mathrm{~mol} / \mathrm{m}^{3}\right)$ and $\zeta_{2}^{r}=f\left(\bar{C}_{1}, \bar{C}_{2}=37.71 \mathrm{~mol} / \mathrm{m}^{3}\right)$ presented in Figure 2 and following data: $L_{p}=4.9 \times 10^{-12} \mathrm{~m}^{3} / \mathrm{Ns}, \sigma_{1}=0.068, \sigma_{2}=0.025, \omega_{11}=0.8 \times 10^{-9} \mathrm{~mol} / \mathrm{Ns}, \omega_{12}=0.81$ $\times 10^{-13} \mathrm{~mol} / \mathrm{Ns}, \omega_{22}=1.43 \times 10^{-9} \mathrm{~mol} / \mathrm{Ns}, \omega_{21}=1.63 \times 10^{-12} \mathrm{~mol} / \mathrm{Ns}, \bar{C}_{1}=2.79 \div 21.67 \mathrm{~mol} / \mathrm{m}^{3}$ and $\bar{C}_{2}=37.71 \mathrm{~mol} / \mathrm{m}^{3}$ were used. The results of calculating these coefficients are presented in Figures $4-10$.

The Graphs 1A and 1B in Figure 4 illustrating the dependencies $R_{11}^{A}=f\left(\bar{C}_{1}, \bar{C}_{2}=37.71 \mathrm{~mol} / \mathrm{m}^{3}\right)$ and $R_{11}^{B}=f\left(\bar{C}_{1}, \bar{C}_{2}=37.71 \mathrm{~mol} / \mathrm{m}^{3}\right)$ were obtained for the Configurations $\mathrm{A}$ and $\mathrm{B}$ of the membrane system. The value of coefficient $R_{11}^{A}$ increases initially nonlinearly from $R_{11}^{A}=2.57 \times 10^{11} \mathrm{Ns} / \mathrm{m}^{3}$ (for $\bar{C}_{1}=1.44 \mathrm{~mol} / \mathrm{m}^{3}$ ) to $R_{11}^{A}=2.89 \times 10^{11} \mathrm{Ns} / \mathrm{m}^{3}$ (for $\bar{C}_{1}=7.56 \mathrm{~mol} / \mathrm{m}^{3}$ ) and next increases nonlinearly to $R_{11}^{A}=16.45 \times 10^{11} \mathrm{Ns} / \mathrm{m}^{3}$ (for $\left.\bar{C}_{1}=14.59 \mathrm{~mol} / \mathrm{m}^{3}\right)$. For $\bar{C}_{1}>16.45 \mathrm{~mol} / \mathrm{m}^{3} R_{11}^{A}$ increases approximately linearly and for $\bar{C}_{1}=21.67 \mathrm{~mol} / \mathrm{m}^{3}$ and $\bar{C}_{2}=37.71 \mathrm{~mol} / \mathrm{m}^{3}$ achieves the value $R_{11}^{A}=19.2 \times 10^{11} \mathrm{Ns} / \mathrm{m}^{3}$. The value of coefficient $R_{11}^{B}$ initially increases linearly from $R_{11}^{B}=11.47 \times 10^{11} \mathrm{Ns} / \mathrm{m}^{3}$ (for $\bar{C}_{1}=1.44 \mathrm{~mol} / \mathrm{m}^{3}$ ) to $R_{11}^{A}=13.22 \times 10^{11} \mathrm{Ns} / \mathrm{m}^{3}$ (for $\bar{C}_{1}=5.41 \mathrm{~mol} / \mathrm{m}^{3}$ ) and next decreases almost linearly from $R_{11}^{B}=12.86 \times 10^{11} \mathrm{Ns} / \mathrm{m}^{3}$ (for $\bar{C}_{1}=6.57 \mathrm{~mol} / \mathrm{m}^{3}$ ) to $R_{11}^{B}=4.14 \times 10^{11} \mathrm{Ns} / \mathrm{m}^{3}$ (for $\bar{C}_{1}=8.74 \mathrm{~mol} / \mathrm{m}^{3}$ ). Besides $R_{11}{ }^{A}=R_{11}^{B}=3.67 \times 10^{11} \mathrm{Ns} / \mathrm{m}^{3}$ (for $\bar{C}_{1}=9.24 \mathrm{~mol} / \mathrm{m}^{3}$ ). For $\bar{C}_{1}>12.72 \mathrm{~mol} / \mathrm{m}^{3} R_{11}^{A}$ increases approximately linearly and for $\bar{C}_{1}=21.67 \mathrm{~mol} / \mathrm{m}^{3}$ achieves the value $R_{11}^{A}=3.04 \times 10^{11} \mathrm{Ns} / \mathrm{m}^{3}$. For homogeneous solutions $R_{11}^{A}=R_{11}^{B}=R_{11}$ increase linearly from $R_{11}=2.3 \times 10^{11} \mathrm{Ns} / \mathrm{m}^{3}$ (for $\bar{C}_{1}=1.44 \mathrm{~mol} / \mathrm{m}^{3}$ ) to $R_{11}=2.53 \times 10^{11} \mathrm{Ns} / \mathrm{m}^{3}$ (for $\bar{C}_{1}=21.67 \mathrm{~mol} / \mathrm{m}^{3}$ ). Besides, it follows from this figure that for $\bar{C}_{1}<9.24 \mathrm{~mol} / \mathrm{m}^{3} R_{11}^{A}<R_{11}^{B}$ and for $\bar{C}_{1}>9.24 \mathrm{~mol} / \mathrm{m}^{3} R_{11}^{A}>R_{11}^{B}$. 


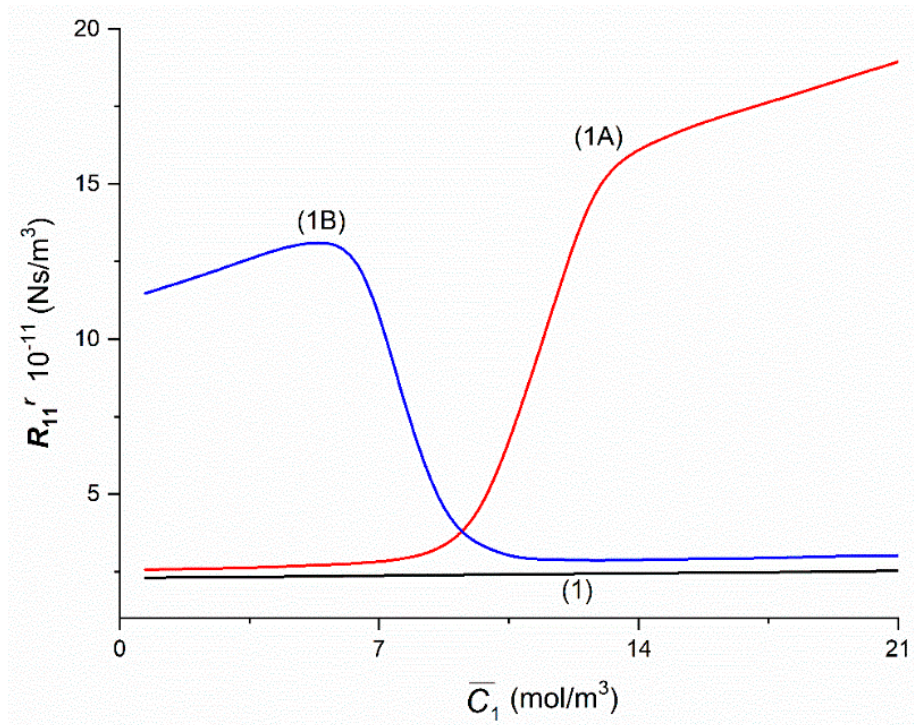

Figure 4. The graphic illustration of the dependences: $R_{i j}^{r}=f\left(\bar{C}_{1}, \bar{C}_{2}=37.71 \mathrm{~mol} / \mathrm{m}^{3}\right),(i, j \in\{1,2,3\}$ and $r=\mathrm{A}$, $B)$ for the glucose in aqueous ethanol solution in conditions of concentration polarization for Configurations $\mathrm{A}$ and $\mathrm{B}$ of the membrane system: Curve $1 \mathrm{~A}$ - for $R_{11}^{A}$, Curve $1 \mathrm{~B}$ - for $R_{11}^{B}$ and Line 1 - for $R_{11}$.

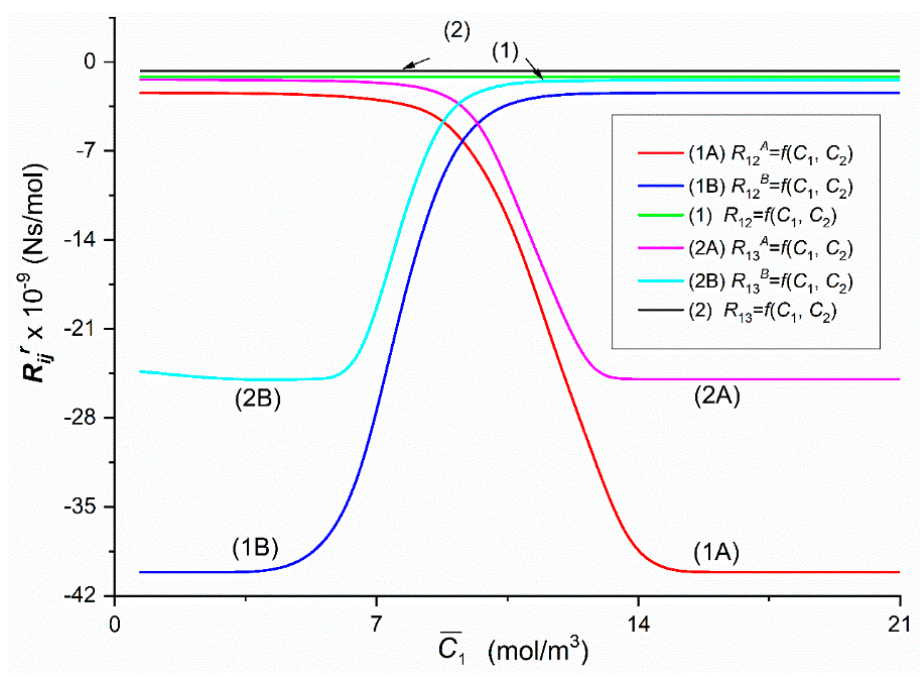

Figure 5. The graphic illustration of the dependences: $R_{i j}^{r}=f\left(\bar{C}_{1}, \bar{C}_{2}=37.71 \mathrm{~mol} / \mathrm{m}^{3}\right),(i, j \in\{1,2,3\}$ and $r=\mathrm{A}, \mathrm{B})$ for the glucose in aqueous ethanol solution in conditions of concentration polarization for Configurations $\mathrm{A}$ and $\mathrm{B}$ of the membrane system: Curve $1 \mathrm{~A}$-for $R_{12^{\prime}}^{A}$ Curve $2 \mathrm{~A}$-for $R_{13^{\prime}}^{A}$ Curve $1 \mathrm{~B}$-for $R_{12}^{B}$, Curve $2 \mathrm{~B}$ - for $R_{13}^{B}$, Line 1 -for $R_{12}$ and Line 2 -for $R_{13}$.

The Graphs 1A, 1B, 2A and 2B illustrating dependencies $R_{12}^{A}=f\left(\bar{C}_{1}, \bar{C}_{2}=37.71 \mathrm{~mol} / \mathrm{m}^{3}\right), R_{12}^{B}$ $=f\left(\bar{C}_{1}, \bar{C}_{2}=37.71 \mathrm{~mol} / \mathrm{m}^{3}\right), R_{21}^{A}=f\left(\bar{C}_{1}, \bar{C}_{2}=37.71 \mathrm{~mol} / \mathrm{m}^{3}\right)$ and $R_{21}^{B}=f\left(\bar{C}_{1}, \bar{C}_{2}=37.71 \mathrm{~mol} / \mathrm{m}^{3}\right)$ presented in Figure 5, were obtained suitably for Configurations A and B of the membrane system, respectively. In the case of Configuration $\mathrm{A}$, the value of coefficients $R_{12}^{A}$ and $R_{13}^{A}$ decreases nonlinearly from $R_{12}^{A}=-2.41 \times 10^{9} \mathrm{Ns} / \mathrm{mol}$ and $R_{13}^{A}=-1.41 \times 10^{9} \mathrm{Ns} / \mathrm{mol}\left(\right.$ for $\left.\bar{C}_{1}=5.41 \mathrm{~mol} / \mathrm{m}^{3}\right)$ to $R_{12}^{A}=-39.95 \times$ $10^{-9} \mathrm{Ns} / \mathrm{mol}$ (for $\bar{C}_{1}=14.59 \mathrm{~mol} / \mathrm{m}^{3}$ ) and to $R_{13}^{A}=-24.76 \times 10^{9} \mathrm{Ns} / \mathrm{mol}\left(\right.$ for $\bar{C}_{1}=13.66 \mathrm{~mol} / \mathrm{m}^{3}$ ). $R_{12}^{A}$ (for $\bar{C}_{1} \geq 15.51 \mathrm{~mol} / \mathrm{m}^{3}$ ) and $R_{13}^{A}$ (for $\bar{C}_{1} \geq 12.72 \mathrm{~mol} / \mathrm{m}^{3}$ ) are constant and amounts to $R_{12}^{A}=-40.15 \times$ $10^{9} \mathrm{Ns} / \mathrm{mol}$ and $R_{13}^{A}=-24.95 \times 10^{9} \mathrm{Ns} / \mathrm{mol}$, respectively. The value of coefficients $R_{12}^{B}$ and $R_{13}^{B}$ increases nonlinearly from $R_{12}^{B}=-40.15 \times 10^{9} \mathrm{Ns} / \mathrm{mol}$ and $R_{13}^{B}=-24.34 \times 10^{-9} \mathrm{Ns} / \mathrm{mol}\left(\right.$ for $\bar{C}_{1}=0.69 \mathrm{~mol} / \mathrm{m}^{3}$ ) to $R_{12}^{B}=-2.46 \times 10^{9} \mathrm{Ns} / \mathrm{mol}$ and $R_{13}^{B}=R_{21}^{B}=-1.43 \times 10^{9} \mathrm{Ns} / \mathrm{mol}\left(\right.$ for $\bar{C}_{1}=12.72 \mathrm{~mol} / \mathrm{m}^{3}$ ). For $\bar{C}_{1}>13.66$ $\mathrm{mol} / \mathrm{m}^{3}, R_{12}^{B}$ and $R_{21}^{B}$ are constant and amounts to $R_{12}^{B}=-2.41 \times 10^{-9} \mathrm{Ns} / \mathrm{mol}$ and $R_{13}^{B}=-1.39 \times 10^{9}$ 
$\mathrm{Ns} / \mathrm{mol}$. For $\bar{C}_{1}=9.24 \mathrm{~mol} / \mathrm{m}^{3}$ and $\bar{C}_{2}=37.71 \mathrm{~mol} / \mathrm{m}^{3} R_{12}^{A}=R_{12}^{B}=-6.0 \times 10^{9} \mathrm{Ns} / \mathrm{mol}$ and $R_{13}^{A}=R_{13}^{B}=$ $-3.0 \times 10^{9} \mathrm{Ns} / \mathrm{mol}$. Besides, for $\bar{C}_{1}<9.24 \mathrm{~mol} / \mathrm{m}^{3} R_{12}^{A}>R_{12}^{B}$ and $R_{13}^{A}>R_{13}^{B}$. For $\bar{C}_{1}>9.24 \mathrm{~mol} / \mathrm{m}^{3} R_{12}^{A}<$ $R_{12}^{B}$ and $R_{13}^{A}<R_{13}^{B}$. For homogeneous solutions $R_{12}^{A}=R_{12}^{B}=R_{12}=-1.16 \times 10^{9} \mathrm{Ns} / \mathrm{mol}<R_{13}^{A}=R_{13}^{B}=$ $R_{13}=-0.68 \times 10^{9} \mathrm{Ns} / \mathrm{mol}$ in whole range of studied $\bar{C}_{1}$ (Lines 1 and 2 ).

The Graphs 1A, 1B, 2A and 2B present dependencies $R_{12}^{A}=f\left(\bar{C}_{1}, \bar{C}_{2}=37.71 \mathrm{~mol} / \mathrm{m}^{3}\right), R_{12}^{B}=f\left(\bar{C}_{1}\right.$, $\left.\bar{C}_{2}=37.71 \mathrm{~mol} / \mathrm{m}^{3}\right), R_{21}^{A}=f\left(\bar{C}_{1}, \bar{C}_{2}=37.71 \mathrm{~mol} / \mathrm{m}^{3}\right)$ and $R_{21}^{B}=f\left(\bar{C}_{1}, \bar{C}_{2}=37.71 \mathrm{~mol} / \mathrm{m}^{3}\right)$ presented in Figure 5, were obtained suitably for Configurations $A$ and $B$ of the membrane system, respectively. In the case of Configuration $\mathrm{A}$, the value of coefficients $R_{12}^{A}$ and $R_{13}^{A}$ decreases nonlinearly from $R_{12}^{A}$ $=-2.41 \times 10^{9} \mathrm{Ns} / \mathrm{mol}$ and $R_{13}^{A}=-1.41 \times 10^{9} \mathrm{Ns} / \mathrm{mol}\left(\right.$ for $\left.\bar{C}_{1}=5.41 \mathrm{~mol} / \mathrm{m}^{3}\right)$ to $R_{12}^{A}=-39.95 \times 10^{-9}$ $\mathrm{Ns} / \mathrm{mol}$ (for $\bar{C}_{1}=14.59 \mathrm{~mol} / \mathrm{m}^{3}$ ) and to $R_{13}^{A}=-24.76 \times 10^{9} \mathrm{Ns} / \mathrm{mol}$ (for $\bar{C}_{1}=13.66 \mathrm{~mol} / \mathrm{m}^{3}$ ). $R_{12}^{A}$ (for $\bar{C}_{1} \geq 15.51 \mathrm{~mol} / \mathrm{m}^{3}$ ) and $R_{13}^{A}$ (for $\bar{C}_{1} \geq 12.72 \mathrm{~mol} / \mathrm{m}^{3}$ ) are constant and amounts to $R_{12}^{A}=-40.15 \times 10^{9}$ $\mathrm{Ns} / \mathrm{mol}$ and $R_{13}^{A}=-24.95 \times 10^{9} \mathrm{Ns} / \mathrm{mol}$, respectively. The value of coefficients $R_{12}^{B}$ and $R_{13}^{B}$ increases nonlinearly from $R_{12}^{B}=-40.15 \times 10^{9} \mathrm{Ns} / \mathrm{mol}$ and $R_{13}^{B}=-24.34 \times 10^{-9} \mathrm{Ns} / \mathrm{mol}\left(\right.$ for $\bar{C}_{1}=0.69 \mathrm{~mol} / \mathrm{m}^{3}$ ) to $R_{12}^{B}=-2.46 \times 10^{9} \mathrm{Ns} / \mathrm{mol}$ and $R_{13}^{B}=R_{21}^{B}=-1.43 \times 10^{9} \mathrm{Ns} / \mathrm{mol}\left(\right.$ for $\bar{C}_{1}=12.72 \mathrm{~mol} / \mathrm{m}^{3}$ ). For $\bar{C}_{1}>13.66$ $\mathrm{mol} / \mathrm{m}^{3}, R_{12}^{B}$ and $R_{21}^{B}$ are constant and amounts to $R_{12}^{B}=-2.41 \times 10^{-9} \mathrm{Ns} / \mathrm{mol}$ and $R_{13}^{B}=-1.39 \times 10^{9}$ $\mathrm{Ns} / \mathrm{mol}$. For $\bar{C}_{1}=9.24 \mathrm{~mol} / \mathrm{m}^{3}$ and $\bar{C}_{2}=37.71 \mathrm{~mol} / \mathrm{m}^{3} R_{12}^{A}=R_{12}^{B}=-6.0 \times 10^{9} \mathrm{Ns} / \mathrm{mol}$ and $R_{13}^{A}=R_{13}^{B}=$ $-3.0 \times 10^{9} \mathrm{Ns} / \mathrm{mol}$. Besides, for $\bar{C}_{1}<9.24 \mathrm{~mol} / \mathrm{m}^{3} R_{12}^{A}>R_{12}^{B}$ and $R_{13}^{A}>R_{13}^{B}$. For $\bar{C}_{1}>9.24 \mathrm{~mol} / \mathrm{m}^{3} R_{12}^{A}<$ $R_{12}^{B}$ and $R_{13}^{A}<R_{13}^{B}$. For homogeneous solutions $R_{12}^{A}=R_{12}^{B}=R_{12}=-1.16 \times 10^{9} \mathrm{Ns} / \mathrm{mol}<R_{13}^{A}=R_{13}^{B}=$ $R_{13}=-0.68 \times 10^{9} \mathrm{Ns} / \mathrm{mol}$ in whole range of studied $\bar{C}_{1}$ (Lines 1 and 2 ).

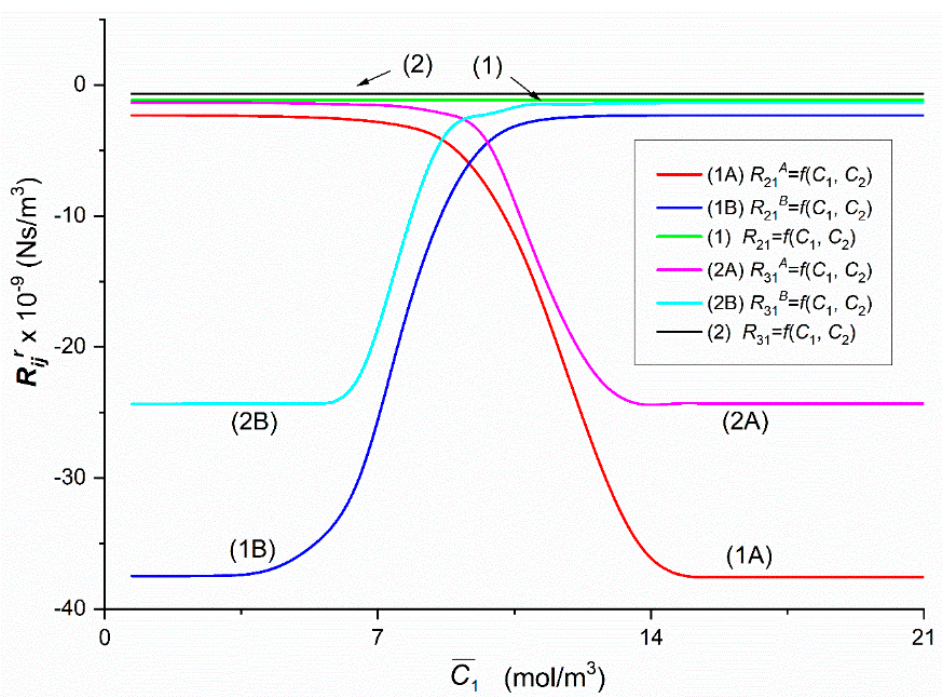

Figure 6. The graphic illustration of the dependences: $R_{i j}^{r}=f\left(\bar{C}_{1}, \bar{C}_{2}=37.71 \mathrm{~mol} / \mathrm{m}^{3}\right),(i, j \in\{1,2,3\}$ and $r=\mathrm{A}, \mathrm{B})$ for the glucose in aqueous ethanol solution in conditions of concentration polarization for Configurations $\mathrm{A}$ and $\mathrm{B}$ of the membrane system: Curve $1 \mathrm{~A}-$ for $R_{21}^{A}$, Curve $2 \mathrm{~A}$-for $R_{31}^{A}$, Curve $1 \mathrm{~B}$-for $R_{21}^{B}$, Curve 2B-for $R_{31}^{B}$, Line 1 -for $R_{21}$ and Line 2-for $R_{31}$.

Graphs 1A, 1B, 2A and 2B illustrating dependencies $R_{21}^{A}=f\left(\bar{C}_{1}, \bar{C}_{2}=37.71 \mathrm{~mol} / \mathrm{m}^{3}\right), R_{21}^{B}=f\left(\bar{C}_{1}\right.$, $\left.\bar{C}_{2}=37.71 \mathrm{~mol} / \mathrm{m}^{3}\right), R_{31}^{A}=f\left(\bar{C}_{1}, \bar{C}_{2}=37.71 \mathrm{~mol} / \mathrm{m}^{3}\right)$ and $R_{31}^{B}=f\left(\bar{C}_{1}, \bar{C}_{2}=37.71 \mathrm{~mol} / \mathrm{m}^{3}\right)$, presented in Figure 6, were obtained suitably for Configurations A and B of the membrane system, respectively. The dependencies shown in this figure are similar to the dependencies shown in Figure 5. 


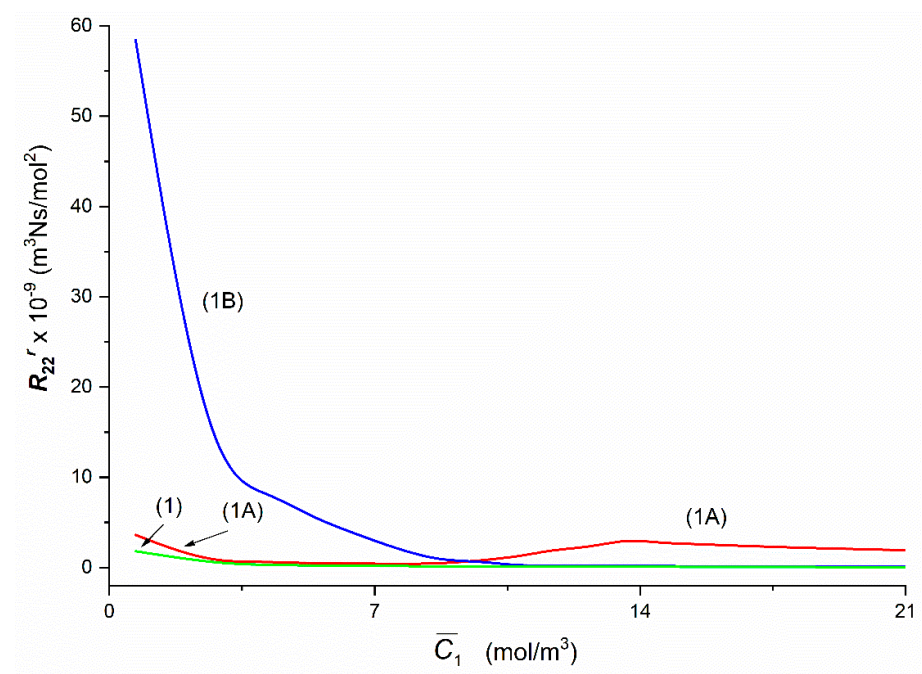

Figure 7. The graphic illustration of the dependences: $R_{22}^{r}=f\left(\bar{C}_{1}, \bar{C}_{2}=37.71 \mathrm{~mol} / \mathrm{m}^{3}\right),(r=\mathrm{A}, \mathrm{B})$ for the glucose in aqueous ethanol solution in conditions of concentration polarization for Configurations $\mathrm{A}$ and $\mathrm{B}$ of the membrane system: Curve $1 \mathrm{~A}$-for $R_{22}^{A}$, Curve $1 \mathrm{~B}$-for $R_{22}^{B}$ and Line 1 -for $R_{22}$.

The Graphs $1 \mathrm{~A}$ and $1 \mathrm{~B}$, illustrating the dependencies $R_{22}^{A}=f\left(\bar{C}_{1}, \bar{C}_{2}=37.71 \mathrm{~mol} / \mathrm{m}^{3}\right)$ and $R_{22}^{B}=$ $f\left(\bar{C}_{1}, \bar{C}_{2}=37.71 \mathrm{~mol} / \mathrm{m}^{3}\right)$, presented in Figure 7 , were obtained for the Configurations $\mathrm{A}$ and $\mathrm{B}$ of the membrane system. Curves 1 and $1 \mathrm{~B}$ illustrate the dependencies $R_{22}=f\left(\bar{C}_{1}, \bar{C}_{2}=37.71 \mathrm{~mol} / \mathrm{m}^{3}\right)$ and $R_{22}^{B}=f\left(\bar{C}_{1}, \bar{C}_{2}=37.71 \mathrm{~mol} / \mathrm{m}^{3}\right)$ are hyperbolas. In turn, Curve $1 \mathrm{~A}$, illustrating the dependence $R_{22}^{A}=$ $f\left(\bar{C}_{1}, \bar{C}_{2}\right)$, is an irregular curve: initially it decreases nonlinearly from $R_{22}^{A}=3.62 \times 10^{9} \mathrm{~m}^{3} \mathrm{Ns} / \mathrm{mol}^{2}$ (for $\bar{C}_{1}=1.44 \mathrm{~mol} / \mathrm{m}^{3}$ ) to $R_{22}^{A}=0.43 \times 10^{9} \mathrm{~m}^{3} \mathrm{Ns} / \mathrm{mol}^{2}$ (for $\bar{C}_{1}=7.68 \mathrm{~mol} / \mathrm{m}^{3}$ ) and then grows nonlinearly to $R_{22}^{A}=2.95 \times 10^{9} \mathrm{~m}^{3} \mathrm{Ns} / \mathrm{mol}^{2}$ (for $\bar{C}_{1}=13.66 \mathrm{~mol} / \mathrm{m}^{3}$ ). For $\bar{C}_{1}>13.66 \mathrm{~mol} / \mathrm{m}^{3} R_{22}^{A}$ decreases linearly to $R_{22}^{A}=1.86 \times 10^{9} \mathrm{~m}^{3} \mathrm{Ns} / \mathrm{mol}^{2}$ (for $\bar{C}_{1}=21.67 \mathrm{~mol} / \mathrm{m}^{3}$ ). In turn for $\bar{C}_{1}=9.24 \mathrm{~mol} / \mathrm{m}^{3} R_{22}^{A}=R_{22}^{B}=0.64 \times$ $10^{9} \mathrm{~m}^{3} \mathrm{Ns} / \mathrm{mol}^{2}$, while for $\bar{C}_{1}<9.24 \mathrm{~mol} / \mathrm{m}^{3} R_{22}^{A}<R_{22}^{B}$ and for $\bar{C}_{1}>9.24 \mathrm{~mol} / \mathrm{m}^{3} R_{22}^{A}>R_{22}^{B}$.

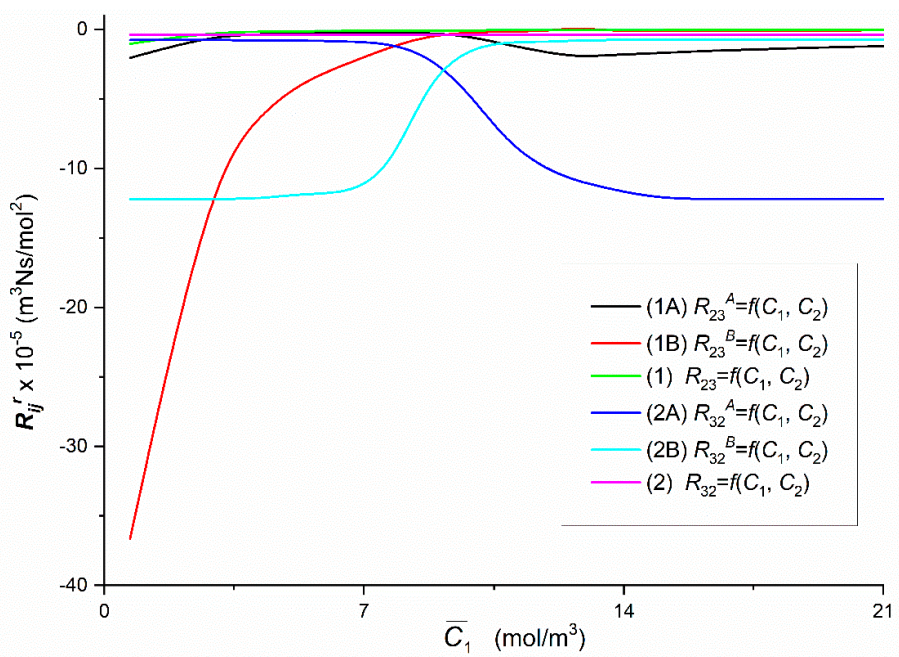

Figure 8. The graphic illustration of the dependences: $R_{i j}^{r}=f\left(\bar{C}_{1}, \bar{C}_{2}=37.71 \mathrm{~mol} / \mathrm{m}^{3}\right),(i, j \in\{1,2,3\}$ and $r=\mathrm{A}, \mathrm{B})$ for the glucose in aqueous ethanol solution in conditions of concentration polarization for Configurations $\mathrm{A}$ and $\mathrm{B}$ of the membrane system: Curve $1 \mathrm{~A}-$ for $R_{23}^{A}$, Curve $2 \mathrm{~A}-$ for $R_{32}^{A}$ Curve $1 \mathrm{~B}$-for $R_{23}^{B}$ Curve $2 \mathrm{~B}$-for $R_{32}^{B}$ Line 1 -for $R_{23}$ and Line 2 -for $R_{32}$. 


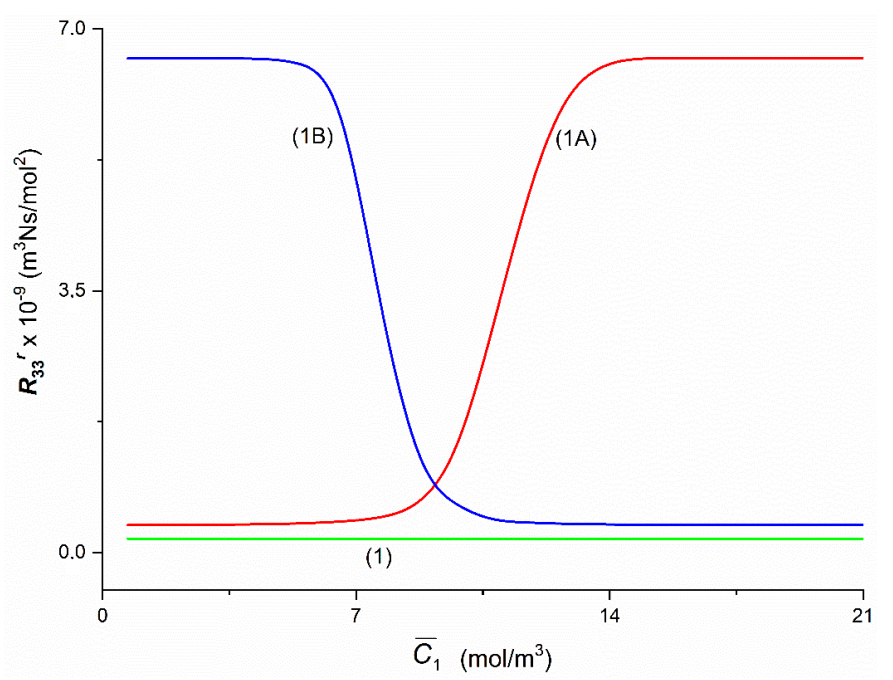

Figure 9. The graphic illustration of the dependence $R_{33}^{r}=f\left(\bar{C}_{1}, \bar{C}_{2}=37.71 \mathrm{~mol} / \mathrm{m}^{3}\right),(r=\mathrm{A}, \mathrm{B})$ for the glucose in aqueous ethanol solution in conditions of concentration polarization for Configurations $\mathrm{A}$ and $\mathrm{B}$ of the membrane system: Curve $1 \mathrm{~A}$ - for $R_{33^{\prime}}^{A}$ Curve $1 \mathrm{~B}$ - for $R_{33}^{B}$ and Line 1 - for $R_{33}$.

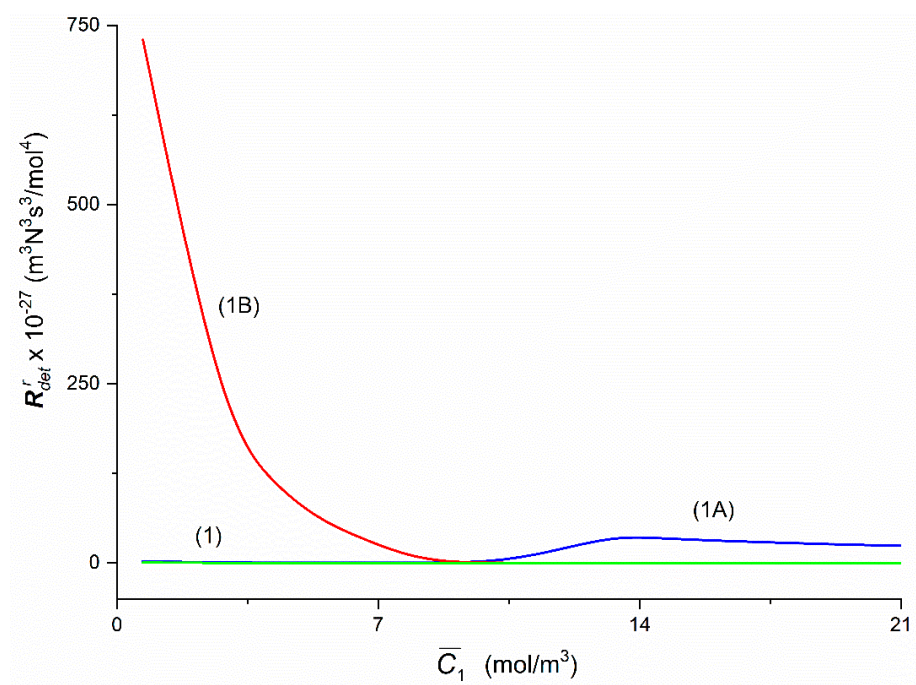

Figure 10. The graphic illustration of the dependence $R_{\text {det }}^{r}=f\left(\bar{C}_{1}, \bar{C}_{2}=37.71 \mathrm{~mol} / \mathrm{m}^{3}\right)(r=A, B)$ for the glucose in aqueous ethanol solution in conditions of concentration polarization for Configuration $\mathrm{A}$ $\left(R_{\text {det }^{\prime}}^{A}\right.$ Curve 1A) and B ( $R_{\text {det }^{\prime}}^{B}$ Curve 1B) of the membrane system. Line 1 illustrates the dependence $R_{\text {det }}$ $=f\left(\bar{C}_{1}, \bar{C}_{2}=\right.$ const. $)$ in conditions of homogeneity of solutions.

Graphs 1A, 1B, 2A and 2B illustrate dependencies $R_{23}^{A}=f\left(\bar{C}_{1}, \bar{C}_{2}=37.71 \mathrm{~mol} / \mathrm{m}^{3}=\right.$ const. $), R_{23}^{B}$ $=f\left(\bar{C}_{1}, \bar{C}_{2}=37.71 \mathrm{~mol} / \mathrm{m}^{3}=\right.$ const. $), R_{32}^{A}=f\left(\bar{C}_{1}, \bar{C}_{2}=37.71 \mathrm{~mol} / \mathrm{m}^{3}=\right.$ const. $)$ and $R_{32}^{B}=f\left(\bar{C}_{1}, \bar{C}_{2}=\right.$ $7.71 \mathrm{~mol} / \mathrm{m}^{3}=$ const.) as presented in Figure 8, were obtained suitably for Configurations A and B of the membrane system, respectively. Curves 1,2 and $1 \mathrm{~B}$ illustrate dependencies $R_{23}=f\left(\bar{C}_{1}, \bar{C}_{2}=\right.$ $37.71 \mathrm{~mol} / \mathrm{m}^{3}=$ const. $), R_{32}=f\left(\bar{C}_{1}, \bar{C}_{2}=37.71 \mathrm{~mol} / \mathrm{m}^{3}=\right.$ const. $)$ and $R_{23}^{B}=f\left(\bar{C}_{1}, \bar{C}_{2}=37.71 \mathrm{~mol} / \mathrm{m}^{3}=\right.$ const.) are hyperbolas. In turn, Curve $1 \mathrm{~A}$ illustrating the dependence $R_{23}^{A}=f\left(\bar{C}_{1}, \bar{C}_{2}=37.71 \mathrm{~mol} / \mathrm{m}^{3}=\right.$ const.) is an irregular curve: initially it grows nonlinearly from $R_{23}^{A}=-2.05 \times 10^{5} \mathrm{~m}^{3} \mathrm{Ns} / \mathrm{mol}^{2}$ (for $\bar{C}_{1}=$ $1.44 \mathrm{~mol} / \mathrm{m}^{3}$ ) to $R_{23}^{A}=-0.23 \times 10^{5} \mathrm{~m}^{3} \mathrm{Ns} / \mathrm{mol}^{2}\left(\right.$ for $\bar{C}_{1}=7.68 \mathrm{~mol} / \mathrm{m}^{3}$ ) and then decreases nonlinearly to $R_{23}^{A}=-1.99 \times 10^{5} \mathrm{~m}^{3} \mathrm{Ns} / \mathrm{mol}^{2}$ (for $\bar{C}_{1}=13.66 \mathrm{~mol} / \mathrm{m}^{3}$ ). For $\bar{C}_{1}>12.71 \mathrm{~mol} / \mathrm{m}^{3} R_{23}^{A}$ increases linearly to $R_{23}^{A}=-1.17 \times 10^{5} \mathrm{~m}^{3} \mathrm{Ns} / \mathrm{mol}^{2}$ (for $\bar{C}_{1}=21.67 \mathrm{~mol} / \mathrm{m}^{3}$ ). For $\bar{C}_{1}=9.24 \mathrm{~mol} / \mathrm{m}^{3}, R_{23}^{A}=R_{23}^{B}=-0.38 \times$ $10^{5} \mathrm{~m}^{3} \mathrm{Ns} / \mathrm{mol}^{2}$, while for $\bar{C}_{1}<9.24 \mathrm{~mol} / \mathrm{m}^{3} R_{23}^{A}>R_{23}^{B}$ and for $\bar{C}_{1}>9.24 \mathrm{~mol} / \mathrm{m}^{3} R_{23}^{A}<R_{23}^{B}$. Curves $2 \mathrm{~A}$ and 2B illustrating respectively the dependence $R_{32}^{A}=f\left(\bar{C}_{1}, \bar{C}_{2}\right)$ and $R_{32}{ }^{B}=f\left(\bar{C}_{1}, \bar{C}_{2}\right)$ intersect at the 
coordinates $\bar{C}_{1}=9.24 \mathrm{~mol} / \mathrm{m}^{3}$ and $R_{32}^{A}=R_{32}^{B}=-2.66 \times 10^{5} \mathrm{~m}^{3} \mathrm{Ns} / \mathrm{mol}^{2}$. For $\bar{C}_{1}<9.24 \mathrm{~mol} / \mathrm{m}^{3} R_{32}^{A}>R_{32}^{B}$ and for $\bar{C}_{1}>9.24 \mathrm{~mol} / \mathrm{m}^{3} R_{32}^{A}<R_{32}^{B}$.

Presented in Figure 9, Graphs 1A and 1B illustrating the dependencies $R_{33}^{A}=f\left(\bar{C}_{1}, \bar{C}_{2}=37.71\right.$ $\left.\mathrm{mol} / \mathrm{m}^{3}\right)$ and $R_{33}^{B}=f\left(\bar{C}_{1}, \bar{C}_{2}=37.71 \mathrm{~mol} / \mathrm{m}^{3}\right)$ were obtained for Configurations $\mathrm{A}$ and $\mathrm{B}$ of the membrane system. The value of coefficient $R_{33}^{A}$ increases nonlinearly from $R_{33}^{A}=0.37 \times 10^{9} \mathrm{~m}^{3} \mathrm{Ns} / \mathrm{mol}^{2}$ (for $\bar{C}_{1}=$ $1.44 \mathrm{~mol} / \mathrm{m}^{3}$ ) to $R_{33}^{A}=6.51 \times 10^{9} \mathrm{~m}^{3} \mathrm{Ns} / \mathrm{mol}^{2}$ (for $\bar{C}_{1}=13.66 \mathrm{~mol} / \mathrm{m}^{3}$ ). For $\bar{C}_{1}>13.66 \mathrm{~mol} / \mathrm{m}^{3} R_{33}^{A}=6.61$ $\times 10^{9} \mathrm{~m}^{3} \mathrm{Ns} / \mathrm{mol}^{2}$ and is constant. The value of coefficient $R_{33}^{B}$ in Configuration B of the membrane system initially is constant and for $\bar{C}_{1}>1.44 \mathrm{~mol} / \mathrm{m}^{3}$ increases nonlinearly from $R_{33}^{B}=6.61 \times 10^{9}$ $\mathrm{m}^{3} \mathrm{Ns} / \mathrm{mol}^{2}$ (for $\bar{C}_{1}=0.69 \mathrm{~mol} / \mathrm{m}^{3}$ ) to $R_{33}^{B}=0.38 \times 10^{9} \mathrm{~m}^{3} \mathrm{Ns} / \mathrm{mol}^{2}$ (for $\bar{C}_{1}=13.66 \mathrm{~mol} / \mathrm{m}^{3}$ ) and next achieves constant value $R_{33}^{B}=0.37 \times 10^{9} \mathrm{~m}^{3} \mathrm{Ns} / \mathrm{mol}^{2}\left(\right.$ for $\left.\bar{C}_{1}>13.66 \mathrm{~mol} / \mathrm{m}^{3}\right)$. Besides $R_{33}^{A}=R_{33}^{B}=0.82$ $\times 10^{9} \mathrm{~m}^{3} \mathrm{Ns} / \mathrm{mol}^{2}$ for $\bar{C}_{1}=9.24 \mathrm{~mol} / \mathrm{m}^{3}$ and $\bar{C}_{2}=37.71 \mathrm{~mol} / \mathrm{m}^{3}$. For homogeneous solutions $R_{33}^{A}=R_{33}^{B}$ $=R_{33}, R_{33}=0.18 \times 10^{9} \mathrm{~m}^{3} \mathrm{Ns} / \mathrm{mol}^{2}$ (for $\bar{C}_{1}=0.69 \mathrm{~mol} / \mathrm{m}^{3}$ ). Besides, it follows from this figure that for $\bar{C}_{1}<9.24 \mathrm{~mol} / \mathrm{m}^{3} R_{33}^{A}<R_{33}^{B}$ and for $\bar{C}_{1}>9.24 \mathrm{~mol} / \mathrm{m}^{3} R_{33}^{A}>R_{33}^{B}$.

The curves presented in Figures 2-9, marked with a number and letters A or B, show that there are transition points from a linear wave to a non-linear wave or vice versa. It is related to the change of the nature of membrane transport from osmotic-diffusion to osmotic-diffusion-convective, or-inversely. The mechanism of this process is as follows. As the concentration of glucose increases at a given concentration of ethanol, the density of the solution, filling the compartment under the membrane in Configuration $B$, increases. If the density of this solution is lower than the density of the solution filling the compartment above the membrane, natural convection occurs in Configuration $\mathrm{B}$, which causes destruction of CBLs, increasing driving forces and increasing the value of the coefficient. The addition of glucose stabilizes the layers and finally eliminates natural convection and changes the nature of transport from osmotic-diffusion-convective to osmotic-diffusion. In Configuration A, the process of creating gravitational convection is in the reverse order. This means that in Configuration $\mathrm{A}$ we have a transition from non-convective to convective, and in Configuration B-from convective to non-convective states. These transitions have a pseudo-phase transition character.

To calculate coefficients $R_{d e t}^{r}$ and $R_{\text {det }}$ the Equations (6) and (8) were used, respectively. The Graphs $1 \mathrm{~A}$ and $1 \mathrm{~B}$ presented in Figure 10 and illustrating the dependencies $R_{\text {det }}^{A}=f\left(\bar{C}_{1}, \bar{C}_{2}=37.71 \mathrm{~mol} / \mathrm{m}^{3}=\right.$ const.) and $R_{\text {det }}^{B}=f\left(\bar{C}_{1}, \bar{C}_{2}=37.71 \mathrm{~mol} / \mathrm{m}^{3}=\right.$ const.) were obtained for the Configurations $\mathrm{A}$ and $\mathrm{B}$ of the membrane system. Curve $1 \mathrm{~B}$ is hyperbolic. In turn, Curve $1 \mathrm{~A}$ is an irregular curve: initially it decreases nonlinearly from $R_{\text {det }}^{A}=2.53 \times 10^{27} \mathrm{~m}^{3} \mathrm{~N}^{3} \mathrm{~s}^{3} / \mathrm{mol}^{4}\left(\right.$ for $\bar{C}_{1}=1.44 \mathrm{~mol} / \mathrm{m}^{3}$ ) to $R_{\text {det }}^{A}=0.7 \times 10^{27}$ $\mathrm{m}^{3} \mathrm{~N}^{3} \mathrm{~s}^{3} / \mathrm{mol}^{4}$ (for $\bar{C}_{1}=7.68 \mathrm{~mol} / \mathrm{m}^{3}$ ) and then grows nonlinearly to $R_{\text {det }}^{A}=36.86 \times 10^{27} \mathrm{~m}^{3} \mathrm{~N}^{3} \mathrm{~s}^{3} / \mathrm{mol}^{4}$ (for $\bar{C}_{1}=13.66 \mathrm{~mol} \mathrm{~m}{ }^{-3}, \bar{C}_{2}=37.71 \mathrm{~mol} / \mathrm{m}^{3}$ ). For $\bar{C}_{1}>13.66 \mathrm{~mol} / \mathrm{m}^{3} R_{\text {det }}^{A}$ decreases linearly to the value of $R_{\text {det }}^{A}=23.24 \times 10^{27} \mathrm{~m}^{3} \mathrm{~N}^{3} \mathrm{~s}^{3} / \mathrm{mol}^{4}$ (for $\bar{C}_{1}=21.67 \mathrm{~mol} / \mathrm{m}^{3}$ ). In turn for $\bar{C}_{1}=9.24 \mathrm{~mol} / \mathrm{m}^{3} R_{\text {det }}^{A}=R_{\text {det }}^{B}=$ $11.20 \times 10^{27} \mathrm{~m}^{3} \mathrm{~N}^{3} \mathrm{~s}^{3} / \mathrm{mol}^{4}$, whereas for $\bar{C}_{1}<9.24 \mathrm{~mol} / \mathrm{m}^{3} R_{\text {det }}^{A}<R_{\text {det }}^{B}$ and for $\bar{C}_{1}>9.24 \mathrm{~mol} / \mathrm{m}^{3} R_{\text {det }}^{A}>R_{\text {det }}^{B}$. For homogeneous solutions, $R_{\text {det }}^{A}=R_{\text {det }}^{B}=R_{\text {det }}$ increase linearly from $R_{\text {det }}=0.63 \times 10^{27} \mathrm{~m}^{3} \mathrm{~N}^{3} \mathrm{~s}^{3} / \mathrm{mol}^{4}$ (for $\bar{C}_{1}=1.44 \mathrm{~mol} / \mathrm{m}^{3}$ ) to $R_{\text {det }}=0.02 \times 10^{27} \mathrm{~m}^{3} \mathrm{~N}^{3} \mathrm{~s}^{3} / \mathrm{mol}^{4}$ (for $\bar{C}_{1}=21.67 \mathrm{~mol} / \mathrm{m}^{3}$ ).

\subsection{Concentration Dependencies of $\xi_{i j}$ and $\xi_{\text {det }}$}

To calculate coefficients $\xi_{i j}=\left(R_{i j}^{A}-R_{i j}^{B}\right) / R_{i j}$ and $\xi_{\operatorname{det}}=\left(\operatorname{det}\left[R^{A}\right]-\operatorname{det}\left[R^{B}\right]\right) / \operatorname{det}[R]$ the Equations (9) and (10) were used, respectively. The graph presented in Figure 11 illustrating the dependencies $\xi_{11}=f\left(\bar{C}_{1}, \bar{C}_{2}=37.71 \mathrm{~mol} / \mathrm{m}^{3}\right)$ was calculated on the basis of Equation (9). In that case the value of coefficient $\xi_{11}$ initially decreases to $\xi_{11}=-4.8$ (for $\bar{C}_{1}=1.44 \mathrm{~mol} / \mathrm{m}^{3}$ ) and next increases nonlinearly to $\xi_{11}=5.41$ (for $\bar{C}_{1}=13.66 \mathrm{~mol} / \mathrm{m}^{3}$ ) and then increases linearly to $\xi_{11}=6.39$ (for $\bar{C}_{1}=21.67 \mathrm{~mol} / \mathrm{m}^{3}$ ). Besides, it follows from this figure that for $\bar{C}_{1}=9.24 \mathrm{~mol} / \mathrm{m}^{3} \xi_{11}=0$ and that $\bar{C}_{1}<9.24 \mathrm{~mol} / \mathrm{m}^{3} \xi_{11}<0$ and for $\bar{C}_{1}>9.24 \mathrm{~mol} / \mathrm{m}^{3} \xi_{11}<0$. 


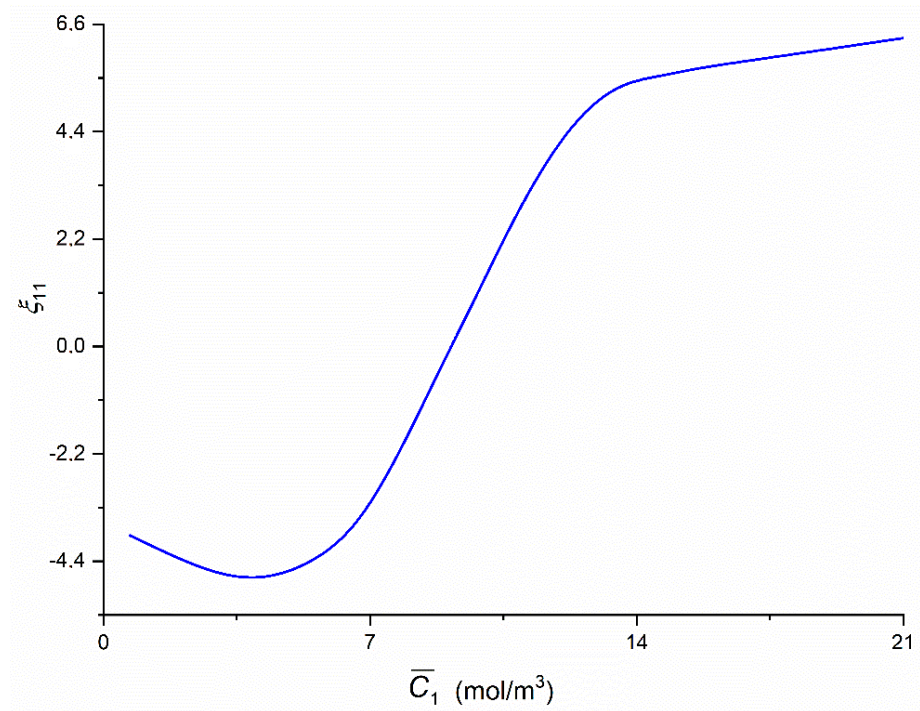

Figure 11. The graphic illustration of the dependence $\xi_{11}=f\left(\bar{C}_{1}, \bar{C}_{2}=37.71 \mathrm{~mol} / \mathrm{m}^{3}\right)$ for the glucose in aqueous ethanol solution.

The graphs presented in Figure 12 which illustrate the dependencies $\xi_{12}=f\left(\bar{C}_{1}, \bar{C}_{2}=37.71\right.$ $\left.\mathrm{mol} / \mathrm{m}^{3}\right), \xi_{13}=f\left(\bar{C}_{1}, \bar{C}_{2}=37.71 \mathrm{~mol} / \mathrm{m}^{3}\right), \xi_{21}=f\left(\bar{C}_{1}, \bar{C}_{2}=37.71 \mathrm{~mol} / \mathrm{m}^{3}\right), \xi_{31}=f\left(\bar{C}_{1}, \bar{C}_{2}=37.71\right.$ $\left.\mathrm{mol} / \mathrm{m}^{3}\right), \xi_{22}=f\left(\bar{C}_{1}, \bar{C}_{2}=37.71 \mathrm{~mol} / \mathrm{m}^{3}\right), \xi_{32}=f\left(\bar{C}_{1}, \bar{C}_{2}=37.71 \mathrm{~mol} / \mathrm{m}^{3}\right), \xi_{23}=f\left(\bar{C}_{1}, \bar{C}_{2}=37.71\right.$ $\left.\mathrm{mol} / \mathrm{m}^{3}\right)$ and $\xi_{33}=f\left(\bar{C}_{1}, \bar{C}_{2}=37.71 \mathrm{~mol} / \mathrm{m}^{3}\right)$ were calculated on the basis of Equation (9). For these graphs, the value of coefficients $\xi_{12}, \xi_{13}, \xi_{21}, \xi_{31}, \xi_{23}$ and $\xi_{32}$ decreases nonlinearly (initially slowly and then faster) from $\xi_{12}>0=$ constant, $\xi_{13}>0=$ constant, $\xi_{21}>0=$ constant, $\xi_{31}>0=$ constant, $\xi_{23}>$ $0=$ constant and $\xi_{32}>0=$ constant $\left(\xi_{21}<\xi_{12}<\xi_{31}<\xi_{13}<\xi_{32}<\xi_{23}\right)$, next $\xi_{12}, \xi_{13}, \xi_{21}, \xi_{31}, \xi_{23}$ and $\xi_{32}$ decreases linearly to $\xi_{12}<0=$ const., $\xi_{13}<0=$ const, $\xi_{21}<0=$ const., $\xi_{31}<0=$ const., $\xi_{23}<0=$ const. and $\xi_{32}<0=$ constant $\left(\xi_{21}>\xi_{32}>\xi_{12}>\xi_{23}>\xi_{31}>\xi_{13}\right)$. It results from this figure that $\xi_{12}=\xi_{13}=$ $\xi_{21}=\xi_{31}=\xi_{23}=\xi_{32}=0$ for $\bar{C}_{1}=9.24 \mathrm{~mol} / \mathrm{m}^{3}$. For these graphs the value of coefficients $\xi_{22}$ and $\xi_{33}$ increases nonlinearly (initially slowly and then faster) from $\xi_{22}<0=$ constant and $\xi_{33}<0=$ constant $\left(\xi_{22}>\xi_{33}\right)$, next $\xi_{22}$ and $\xi_{33}$ increases linearly to $\xi_{22}>0=$ const. and $\xi_{33}>0=$ constant. Besides, it follows from this figure that for $\bar{C}_{1}=9.24 \mathrm{~mol} \mathrm{~m}^{-3}, \xi_{22}=\xi_{33}=0$.

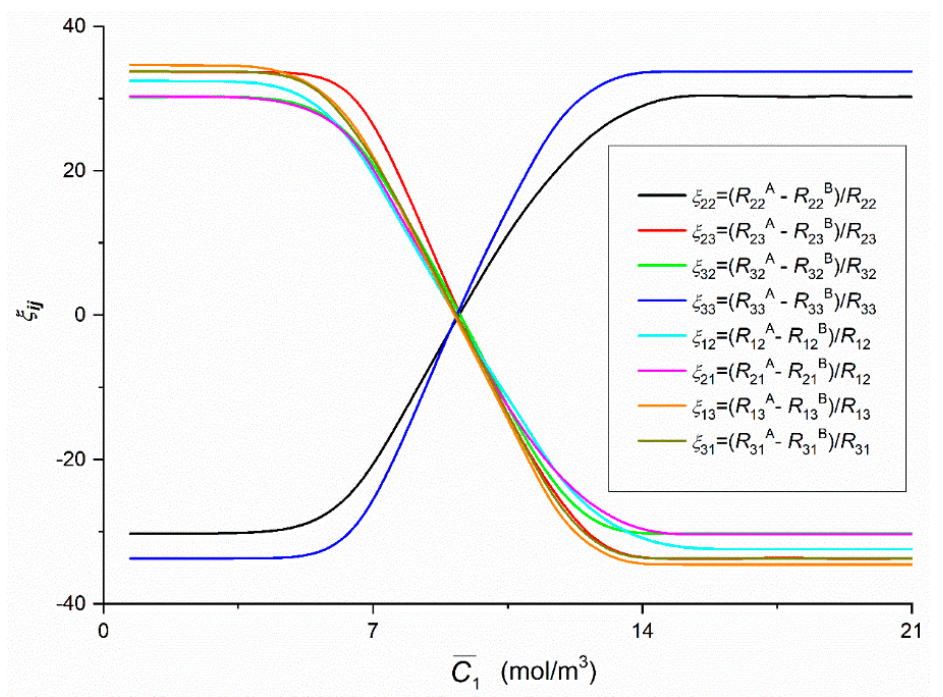

Figure 12. The graphic illustration of the dependence $\xi_{i j}=f\left(\bar{C}_{1}, \bar{C}_{2}=37.71 \mathrm{~mol} / \mathrm{m}^{3}\right)(i, j \in\{1,2,3\}$ and $r=\mathrm{A}, \mathrm{B})$.

The graph presented in Figure 13 illustrating the dependencies $\xi_{\text {det }}=f\left(\bar{C}_{1}, \bar{C}_{2}=37.71 \mathrm{~mol} / \mathrm{m}^{3}=\right.$ const.) was calculated on the basis of Equation (10). In the case of this curve the value of coefficient $\xi_{\text {det }}$ 
initially is constant and amounts $\xi_{\text {det }}=-0.034$ and next increases nonlinearly to $\xi_{\text {det }}=-1148.94$ (for $\bar{C}_{1}=$ $1.44 \mathrm{~mol} / \mathrm{m}^{3}$ ), then increases linearly to $\xi_{\text {det }}=866.38$ (for $\bar{C}_{1}=12.73 \mathrm{~mol} / \mathrm{m}^{3}$ ) and next, nonlinearly to $\xi_{\text {det }}$ $=1148.38$ (for $\bar{C}_{1} \geq 21.67 \mathrm{~mol} / \mathrm{m}^{3}$ ). Besides, it follows from this figure that for $\bar{C}_{1}=9.24 \mathrm{~mol} / \mathrm{m}^{3}, \xi_{\text {det }}=0$. In all cases of the dependencies, $R_{i j}^{r}=f\left(\bar{C}_{1}, \bar{C}_{2}=37.71 \mathrm{~mol} / \mathrm{m}^{3}\right)(i, j \in\{1,2,3\}, r=\mathrm{A}$ or B) and $R_{d e t}^{r}=f\left(\bar{C}_{1}, \bar{C}_{2}=37.71 \mathrm{~mol} / \mathrm{m}^{3}\right),(r=\mathrm{A}$ or B) shown in Figures $5-10$ show clearly that their values are determined by the hydrodynamic conditions in solutions near membrane which separates ternary non-electrolytes with different concentrations. It means that values of these coefficients in concentration polarization conditions are strongly connected with concentrations $\bar{C}_{1}$ and $\bar{C}_{2}$ and configuration of the membrane system. In turn, in the case of mechanical stirring of solutions, the values of these coefficients depend only on concentrations $\bar{C}_{1}$ and $\bar{C}_{2}$. Therefore, for interpretation of calculation results, the combinations of coefficients $R_{i j}^{A}, R_{i j}^{B}$ and $R_{i j}\left(i, j \in\{1,2,3)\right.$ of the same indicators and $R_{d e t}^{A}, R_{\text {det }}^{B}$ and $R_{\text {det }}$ were used. These combinations are presented by Equations (5)-(10). Concentration dependencies of new coefficients facilitate the location of areas differentiated by hydrodynamic conditions in adjacent membrane areas such as diffusion, natural convection-diffusion and natural convection.

Comparison of the results of the tests presented in Figures 4-10 and the results presented in Figures 11-13 results in the signs of the factors $\xi_{i j}$ and $\xi_{\text {det }}$. The results of this comparison are summarized in Table 2.

From the results presented in Figures 4-10, it also appears that the $R_{i j}^{r}$ and $R_{d e t}^{r}(i, j \in\{1,2,3\}$, $r=\mathrm{A}, \mathrm{B})$, have different physical significance. The unit of coefficients $R_{11}^{r}, R_{21}^{r}$ and $R_{31}^{r}$ is $\mathrm{Ns} / \mathrm{m}^{3}$. Therefore, they have the character of flow resistance coefficients (hydraulic resistance). In turn, the unit of coefficients $R_{12}^{r}$ i $R_{13}^{r}$ is Ns/mol, what makes them coefficients of flow resistance of dissolved substances (diffusion resistance). The unit of coefficients $R_{22}^{r}, R_{23}^{r}, R_{32}^{r}$ and $R_{33}^{r}$ is $\mathrm{m}^{3} \mathrm{Ns} / \mathrm{mol}^{2}$. This unit is a measure of the ratio of diffusion resistance to concentration. The unit of the coefficient $R_{d e t}^{r}$ is $\mathrm{m}^{3} \mathrm{~N}^{3} \mathrm{~s}^{3} / \mathrm{mol}^{4}$. It corresponds to the ratio of diffusion resistance raised to the power of third and concentration.

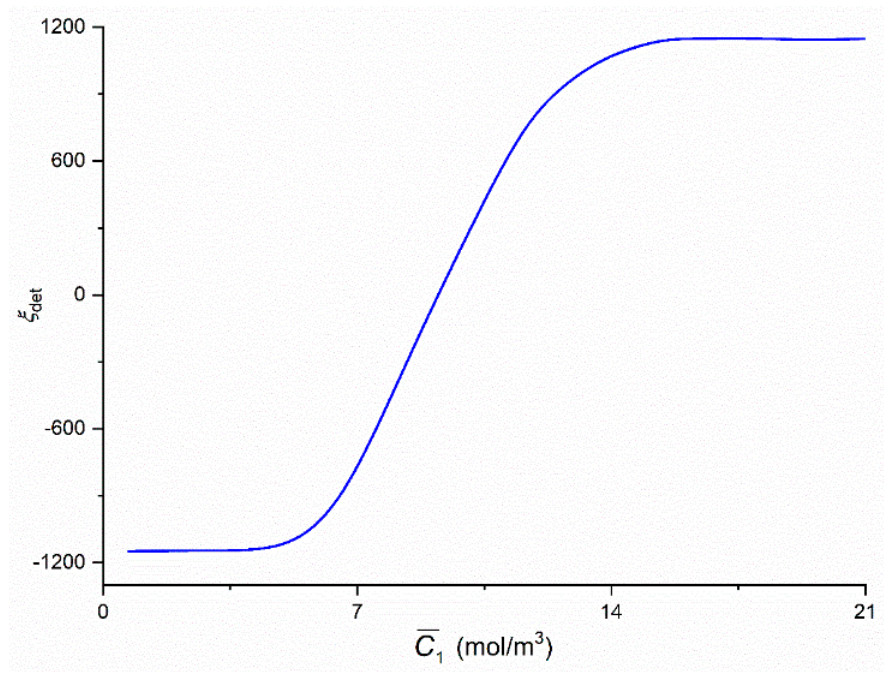

Figure 13. The graphic illustration of the dependence $\xi_{\text {det }}=f\left(\bar{C}_{1}, \bar{C}_{2}=37.71 \mathrm{~mol} / \mathrm{m}^{3}\right)$.

\subsection{Concentration Dependencies of $r_{i j}^{r}, r_{i j}, e_{i j^{\prime}}^{r}, e_{i j}, Q_{R}^{r}$ and $Q_{R}$}

Figures 14-16 show the dependences $r_{i j}^{r}=f\left(\bar{C}_{1}, \bar{C}_{2}=37.71 \mathrm{~mol} / \mathrm{m}^{3}\right)$ and $r_{i j}=f\left(\bar{C}_{1}, \bar{C}_{2}=37.71\right.$ $\left.\mathrm{mol} / \mathrm{m}^{3}\right),(i, j \in\{1,2,3\}$ and $r=\mathrm{A}, \mathrm{B})$ calculated on the basis of Equations (11) and (12) and data presented in Figures 4-9. Figure 14 shows that Curves $1 \mathrm{~A}$ and $1 \mathrm{~B}$ intersect at a point with coordinates: $r_{12}^{A}=r_{12}^{B}=0.36$ and $\bar{C}_{1}=9.15 \mathrm{~mol} / \mathrm{m}^{3}$, and the Curves $2 \mathrm{~A}$ and $2 \mathrm{~B}$ - at a point with coordinates: $r_{21}^{A}=$ $r_{21}^{B}=0.35$ and $\bar{C}_{1}=9.33 \mathrm{~mol} / \mathrm{m}^{3}$. The course of Curves $1 \mathrm{~A}, 1 \mathrm{~B}$ and 1 shows that for $\bar{C}_{1}<9.15 \mathrm{~mol} / \mathrm{m}^{3}$, $r_{12}^{B}>r_{12}^{A}>r_{12}$ and for $\bar{C}_{1}>9.15 \mathrm{~mol} / \mathrm{m}^{3}, r_{12}^{A}>r_{12}^{B}>r_{12}$. Similarly, Curves $2 \mathrm{~A}, 2 \mathrm{~B}$ and 2 show that for 
$\bar{C}_{1}<9.33 \mathrm{~mol} / \mathrm{m}^{3}, r_{21}^{B}>r_{21}^{A}>r_{21}$ and for $\bar{C}_{1}>9.33 \mathrm{~mol} / \mathrm{m}^{3}, r_{21}^{A}>r_{21}^{B}>r_{21}$. Curves $1 \mathrm{~B}$ and $2 \mathrm{~B}$ have maxima. The coordinates of the maximum of Curve $1 \mathrm{~B}$ are $r_{12}^{B}=0.48$ and $\bar{C}_{1}=6.53 \mathrm{~mol} / \mathrm{m}^{3}$. In turn, the coordinates of maximum of the $2 \mathrm{~B}$ curve are $r_{21}^{B}=0.46$ and $\bar{C}_{1}=7.14 \mathrm{~mol} / \mathrm{m}^{3}$. This means that the maximum of Curve $1 \mathrm{~B}$ is shifted relative to the maximum of Curve $2 \mathrm{~B}$ vertically by $\left(r_{12}^{B}-r_{21}^{B}\right)=$ 0.02 and horizontally by $\Delta \bar{C}_{1}=0.61 \mathrm{~mol} / \mathrm{m}^{3}$. In addition, Curves $1 \mathrm{~A}$ and $2 \mathrm{~A}$ and Curves $1 \mathrm{~B}$ and $2 \mathrm{~B}$ are shifted relative to each other, except for the point with coordinates $r_{12}^{A}=r_{21}^{A}=0.14$ and $\bar{C}_{1}=2.15$ $\mathrm{mol} / \mathrm{m}^{3}$. This means that for $\bar{C}_{1}<2.15 \mathrm{~mol} / \mathrm{m}^{3} r_{21}^{A}=r_{12}^{A}$ while for $\bar{C}_{1}>2.15 \mathrm{~mol} / \mathrm{m}^{3} r_{12}^{A}=r_{21}^{A}$. Curves $1 \mathrm{~B}$ and $2 \mathrm{~B}$ coincide on the section with coordinates $r_{12}^{B}=r_{21}^{B}=0.48$ and $\bar{C}_{1}=8.03 \mathrm{~mol} / \mathrm{m}^{3}$ and $r_{12}^{B}=r_{21}^{B}$ $=0.33$ and $\bar{C}_{1}=9.47 \mathrm{~mol} / \mathrm{m}^{3}$. For $\bar{C}_{1}<8.03 \mathrm{~mol} / \mathrm{m}^{3}$ and $\bar{C}_{1}>9.47 \mathrm{~mol} / \mathrm{m}^{3}$ the condition $r_{12}^{B}>r_{21}^{B}$ is fulfilled. Curves 1 and 2 show that the condition $r_{12}=r_{21}$ is fulfilled.

Table 2. Relationships between coefficients $R_{i j}^{A}, R_{i j}^{B}, R_{i j}, \xi_{i j}\left(\mathrm{i}, \mathrm{j} \in\{1,2,3\}, R_{\text {det }}^{A}, R_{\text {det }}^{B}\right.$ and $\xi_{\text {det }}$.

\begin{tabular}{|c|c|c|}
\hline \multirow{3}{*}{$R_{11}^{A}>0, R_{11}^{B}>0, R_{11}>0$} & $R_{11}^{A}<R_{11}^{B}, R_{11}<R_{11}^{A}, R_{11}<R_{11}^{B}$ & $\xi_{11}<0$ \\
\hline & $R_{11}^{A}>R_{11}^{B}, R_{11}<R_{11}^{A}, R_{11}<R_{11}^{B}$ & $\xi_{11}>0$ \\
\hline & $R_{11}^{A}=R_{11}^{B}, R_{11}<R_{11}^{A}, R_{11}<R_{11}^{B}$ & $\xi_{11}=0$ \\
\hline \multirow{3}{*}{$R_{12}^{A}<0, R_{12}^{B}<0, R_{12}<0$} & $R_{12}^{A}>R_{12}^{B}, R_{12}>R_{12}^{A}, R_{12}>R_{12}^{B}$ & $\xi_{12}<0$ \\
\hline & $R_{12}^{A}<R_{12}^{B}, R_{12}>R_{12}^{A}, R_{12}>R_{12}^{B}$ & $\xi_{12}>0$ \\
\hline & $R_{12}^{A}=R_{12}^{B}, R_{12}>R_{12}^{A}, R_{12}>R_{12}^{B}$ & $\xi_{12}=0$ \\
\hline \multirow{3}{*}{$R_{21}^{A}<0, R_{21}^{B}<0, R_{21}<0$} & $R_{21}^{A}>R_{21}^{B}, R_{21}>R_{21}^{A}, R_{21}>R_{21}^{B}$ & $\xi_{21}<0$ \\
\hline & $R_{21}^{A}<R_{21}^{B}, R_{21}>R_{21}^{A}, R_{21}>R_{21}^{B}$ & $\xi_{21}>0$ \\
\hline & $R_{21}^{A}=R_{21}^{B}, R_{21}>R_{21}^{A}, R_{21}>R_{21}^{B}$ & $\xi_{21}=0$ \\
\hline \multirow{3}{*}{$R_{13}^{A}<0, R_{13}^{B}<0, R_{13}<0$} & $R_{13}^{A}>R_{13}^{B}, R_{13}>R_{13}^{A}, R_{13}>R_{13}^{B}$ & $\xi_{13}<0$ \\
\hline & $R_{13}^{A}<R_{13^{\prime}}^{B}, R_{13}>R_{13^{\prime}}^{A}, R_{13}>R_{13}^{B}$ & $\xi_{13}>0$ \\
\hline & $R_{13}^{A}=R_{13}^{B}, R_{13}>R_{13}^{A}, R_{13}>R_{13}^{B}$ & $\xi_{13}=0$ \\
\hline \multirow{3}{*}{$R_{31}^{A}<0, R_{31}^{B}<0, R_{31}<0$} & $R_{31}^{A}>R_{31}^{B}, R_{31}>R_{31}^{A}, R_{31}>R_{31}^{B}$ & $\xi_{31}<0$ \\
\hline & $R_{31}^{A}<R_{31}^{B}, R_{31}>R_{31}^{A}, R_{31}>R_{31}^{B}$ & $\xi_{31}>0$ \\
\hline & $R_{31}^{A}=R_{31}^{B}, R_{31}>R_{31}^{A}, R_{31}>R_{31}^{B}$ & $\xi_{31}=0$ \\
\hline \multirow{3}{*}{$R_{22}^{A}>0, R_{22}^{B}>0, R_{22}>0$} & $R_{22}^{A}<R_{22}^{B}, R_{22}<R_{22}^{A}, R_{22}<R_{22}^{B}$ & $\xi_{22}<0$ \\
\hline & $R_{22}^{A}>R_{22}^{B}, R_{22}<R_{22}^{A}, R_{22}<R_{22}^{B}$ & $\xi_{22}>0$ \\
\hline & $R_{22}^{A}=R_{22}^{B}, R_{22}<R_{22}^{A}, R_{22}<R_{22}^{B}$ & $\xi_{22}=0$ \\
\hline \multirow{3}{*}{$R_{23}^{A}<0, R_{23}^{B}<0, R_{23}<0$} & $R_{23}^{A}>R_{23}^{B}, R_{23}>R_{23}^{A}, R_{23}>R_{23}^{B}$ & $\xi_{23}<0$ \\
\hline & $R_{23}^{A}<R_{23}^{B}, R_{23}>R_{23}^{A}, R_{23}>R_{23}^{B}$ & $\xi_{23}>0$ \\
\hline & $R_{23}^{A}=R_{23}^{B}, R_{23}>R_{23}^{A}, R_{23}>R_{23}^{B}$ & $\xi_{23}=0$ \\
\hline \multirow{3}{*}{$R_{32}^{A}<0, R_{32}^{B}<0, R_{32}<0$} & $R_{32}^{A}>R_{32}^{B}, R_{32}>R_{32}^{A}, R_{32}>R_{23}^{B}$ & $\xi_{32}<0$ \\
\hline & $R_{32}^{A}<R_{32}^{B}, R_{32}>R_{32}^{A}, R_{32}>R_{23}^{B}$ & $\xi_{32}>0$ \\
\hline & $R_{32}^{A}=R_{32}^{B}, R_{32}>R_{32}^{A}, R_{32}>R_{23}^{B}$ & $\xi_{32}=0$ \\
\hline \multirow{3}{*}{$R_{33}^{A}>0, R_{33}^{B}>0, R_{33}>0$} & $R_{33}^{A}<R_{33}^{B}, R_{33}<R_{33}^{A}, R_{33}<R_{33}^{B}$ & $\xi_{33}<0$ \\
\hline & $R_{33}^{A}>R_{33}^{B}, R_{33}<R_{33}^{A}, R_{33}<R_{33}^{B}$ & $\xi_{33}>0$ \\
\hline & $R_{33}^{A}=R_{33^{\prime}}^{B}, R_{33}<R_{33^{\prime}}^{A}, R_{33}<R_{33}^{B}$ & $\xi_{33}=0$ \\
\hline \multirow{3}{*}{$R_{\text {det }}^{A}>0, R_{\text {det }}^{B}>0, R_{\text {det }}>0$} & $R_{\text {det }}^{A}<R_{d e t^{\prime}}^{B}, R_{\text {det }}<R_{\text {det }}^{A}, R_{\text {det }}<R_{\text {det }}^{B}$ & $\xi_{\text {det }}<0$ \\
\hline & $R_{d e t}^{A}>R_{d e t^{\prime}}^{B} R_{\text {det }}<R_{d e t^{\prime}}^{A} R_{\text {det }}<R_{d e t}^{B}$ & $\xi_{\text {det }}>0$ \\
\hline & $R_{d e t}^{A}=R_{d e t^{\prime}}^{B} R_{d e t}<R_{d e t^{\prime}}^{A} R_{d e t}<R_{d e t}^{B}$ & $\xi_{\text {det }}=0$ \\
\hline
\end{tabular}




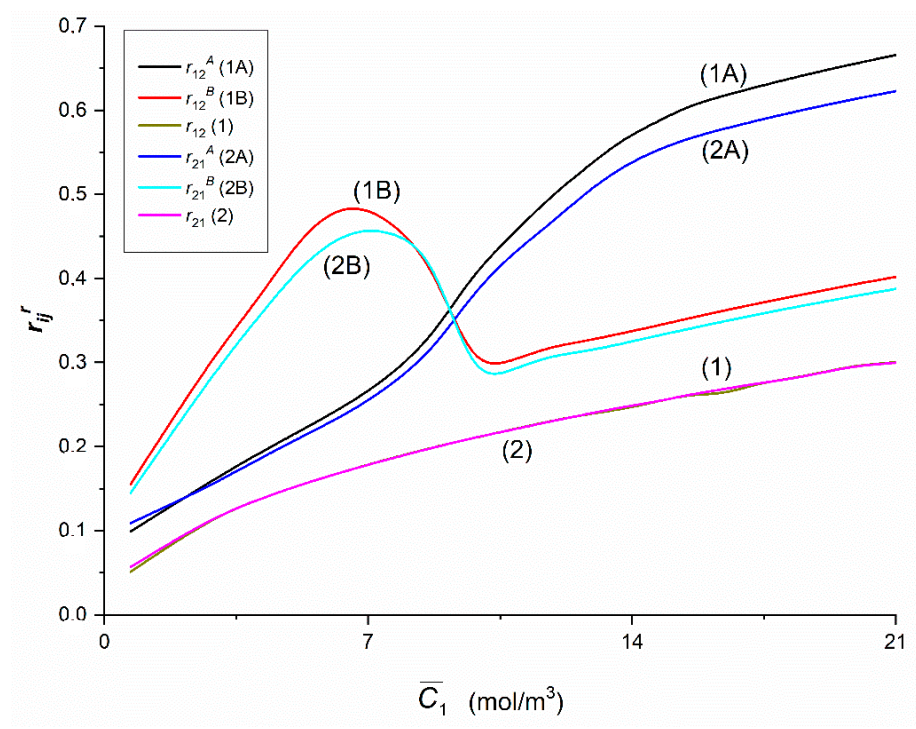

Figure 14. The $r_{i j}^{r}$ and $r_{i j}(i, j \in\{1,2\}, r=\mathrm{A}, \mathrm{B})$ coefficients as functions of glucose concentration.

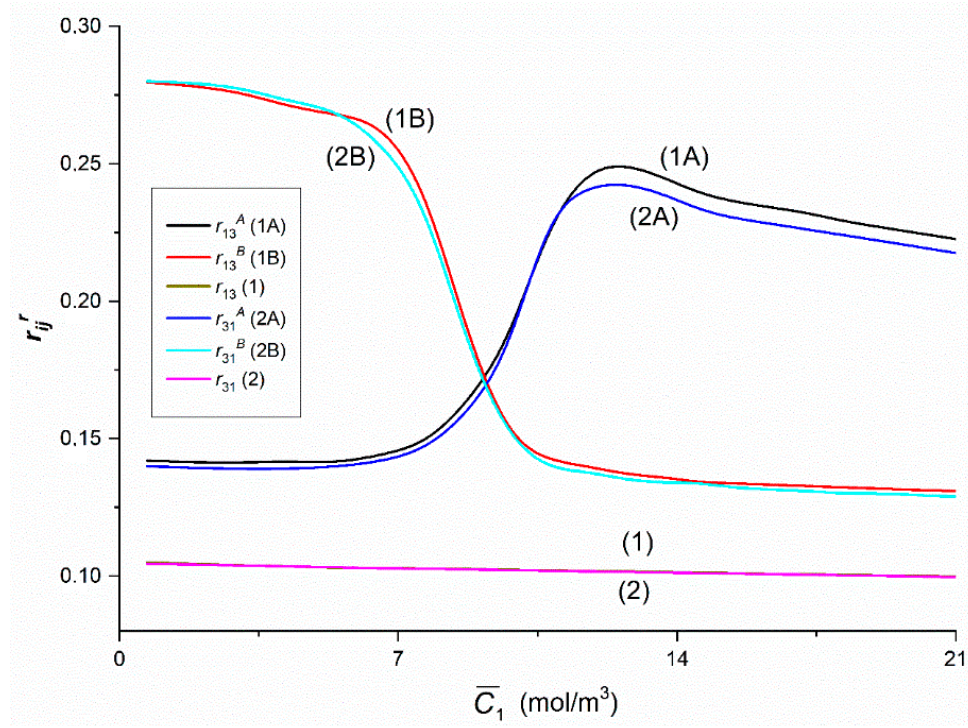

Figure 15. The $r_{i j}^{r}$ and $r_{i j}(i, j \in\{1,3\}, r=\mathrm{A}, \mathrm{B})$ coefficients as functions of glucose concentration.

Figure 15 shows that Curves $1 \mathrm{~A}$ and $1 \mathrm{~B}$ intersect at a point with coordinates $r_{13}^{A}=r_{13}^{B}=0.17$ and $\bar{C}_{1}=9.1 \mathrm{~mol} / \mathrm{m}^{3}$, and Curves $2 \mathrm{~A}$ and $2 \mathrm{~B}$-at a point with coordinates $r_{31}^{A}=r_{31}^{B}=0.17 \mathrm{i} \bar{C}_{1}=9.29 \mathrm{~mol} / \mathrm{m}^{3}$. The course of Curves $1 \mathrm{~A}, 1 \mathrm{~B}$ and 1 shows that for $\bar{C}_{1}<9.1 \mathrm{~mol} / \mathrm{m}^{3}, r_{13}^{B}>r_{13}^{A}>r_{13}$ and for $\bar{C}_{1}>9.1$ $\mathrm{mol} / \mathrm{m}^{3}, r_{13}^{A}>r_{13}^{B}>r_{13}$. Similarly, Curves $2 \mathrm{~A}, 2 \mathrm{~B}$ and 2 show that for $\bar{C}_{1}<9.29 \mathrm{~mol} / \mathrm{m}^{3}, r_{31}^{B}>r_{31}^{A}>r_{31}$ and for $\bar{C}_{1}>9.29 \mathrm{~mol} / \mathrm{m}^{3}, r_{31}^{A}>r_{31}^{B}>r_{31}$. Curves $1 \mathrm{~B}$ and $2 \mathrm{~B}$ overlap in the whole range of $\bar{C}_{1}$ used. Therefore, it can be assumed that $r_{13}^{B}=r_{31}^{B}$. In turn, Curves 1A and 2A do not coincide only beyond the point with the coordinates: $r_{13}^{A}=r_{31}^{A}=0.24$ and $\bar{C}_{1}=11.25 \mathrm{~mol} / \mathrm{m}^{3}$. For $\bar{C}_{1}>11.25 \mathrm{~mol} / \mathrm{m}^{3}, r_{13}^{A}>r_{31}^{A}$. Curves 1 and 2 show that the condition $r_{12}=r_{21}$ is fulfilled.

From the course of curves shown in Figure 16, it follows that $r_{23}^{A}=r_{23}^{B}=r_{23}$ and $r_{32}^{A}=r_{32}^{B}=r_{32}$. Curves 1 and 2 and $1 \mathrm{~A}$ and $2 \mathrm{~B}$ intersect at a point with coordinates $r_{23}=r_{32}=r_{23}^{A}=r_{32}^{B}=0.11 \times 10^{-3}$ and $\bar{C}_{1}=2.33 \mathrm{~mol} / \mathrm{m}^{3}$, while Curves $1 \mathrm{~B}$ and $2 \mathrm{~A}$ - at a point with coordinates $r_{23}^{B}=r_{32}^{A}=0.12$ and $\bar{C}_{1}=$ $2.6 \mathrm{~mol} / \mathrm{m}^{3}$. For $\bar{C}_{1}<2.33 \mathrm{~mol} / \mathrm{m}^{3}, r_{23}=r_{23}^{A}=r_{23}^{B}>r_{32}=r_{32}^{A}=r_{23}^{B}=r_{32}^{B}$ and for $\bar{C}_{1}>2.33 \mathrm{~mol} / \mathrm{m}^{3}, r_{23}=$ $r_{23}^{A}=r_{23}^{B}>r_{32}=r_{32}^{A}=r_{32}^{B}$. As can be seen, the values of the coefficients $r_{i j}, r_{i j^{\prime}}^{r} r_{j i}$ and $r_{j i}^{r}(i, j \in\{2,3\}$ and $r$ 
$=\mathrm{A}, \mathrm{B})$ (Figure 16) are three orders of magnitude smaller than the values of the coefficients $r_{i j}, r_{i j}^{r}, r_{j i}$ and $r_{j i}^{r}(i, j \in\{1,2\}$ and $r=\mathrm{A}, \mathrm{B})$ and $r_{i j}, r_{i j^{\prime}}^{r}, r_{j i}$ and $r_{j i}^{r}(i, j \in\{1,3\}$ and $r=\mathrm{A}, \mathrm{B})$ (Figures 14 and 15).

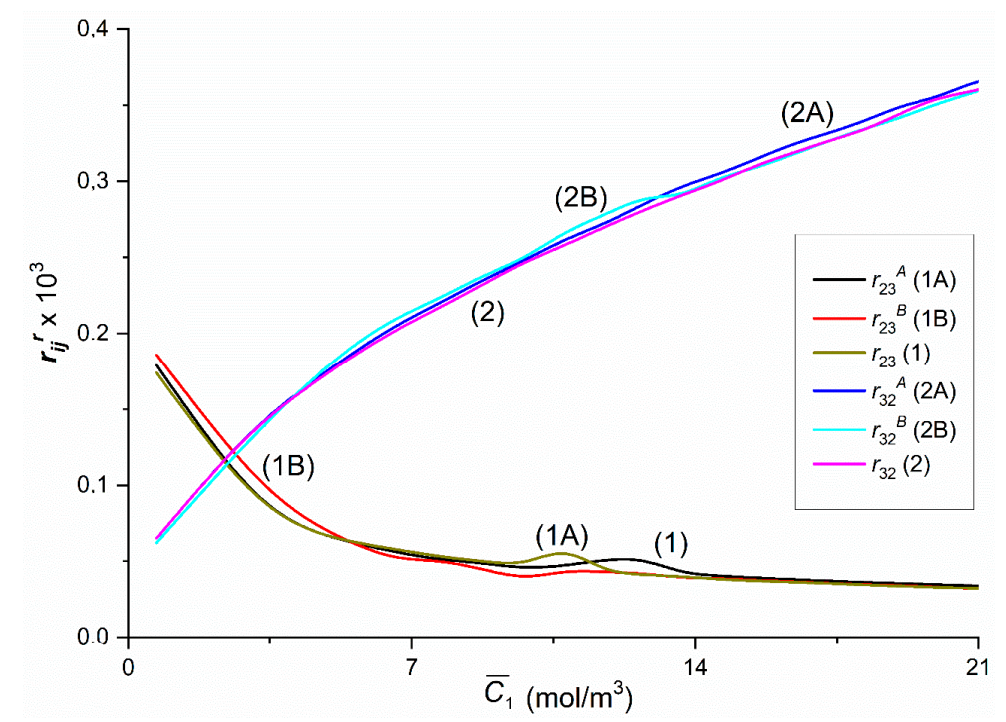

Figure 16. The $r_{i j}^{r}$ and $r_{i j}(i, j \in\{2,3\}, r=\mathrm{A}, \mathrm{B})$ coefficients as functions of glucose concentration.

Figures 14-16 show that Kedem-Caplan relations take the form: $0.05 \leq r_{12}=r_{21} \leq 0.3,0.1 \leq r_{12}^{A} \leq$ $0.67,0.15 \leq r_{12}^{B} \leq 0.48,0.11 \leq r_{21}^{A} \leq 0.63,0.15 \leq r_{21}^{B} \leq 0.46,0.1 \leq r_{13}=r_{31} \leq 0.11,0.14 \leq r_{13}^{A} \leq 0.24,0.13 \leq$ $r_{13}^{B} \leq 0.28,0.11 \leq r_{31}^{A} \leq 0.24,0.13 \leq r_{31}^{B} \leq 0.25,0.03 \times 10^{-3} \leq r_{23}=r_{23}^{A}=r_{23}^{B} \leq 0.18 \times 10^{-3}, 0.06 \times 10^{-3} \leq$ $r_{32}=r_{32}^{A}=r_{32}^{B} \leq 0.36 \times 10^{-3}$. Hence it follows that, $r_{12}^{A} \neq r_{21}^{A}, r_{12}^{B} \neq r_{21}^{B}, r_{13}^{A} \approx r_{31}^{A}, r_{13}^{B}=r_{31}^{B}, r_{23}=r_{23}^{A}=r_{23}^{B}$ $\neq r_{32}=r_{32}^{A}=r_{32}^{B}$. The values of all coupling coefficients presented in Figure 8a,b and Figure 9a fulfilled the conditions $0 \leq r_{i j} \leq 1,0 \leq r_{i j}^{r} \leq 1,0 \leq r_{j i} \leq 1$ and $0 \leq r_{j i}^{r} \leq 1$ determined by Roy Caplan [20].

Graphs in Figures 14 and 15 have characteristic shapes, depending on the configuration of the membrane system and the properties of the solutions. In the case of homogeneous solutions (mechanically stirred solutions-Graphs 1 and 2), the coefficients do not depend on the configuration of the membrane system and are approximately linearly dependent on the concentration of glucose. This means that mechanical stirring of solutions at a sufficiently high speed eliminates CBL creation and causes maximization of fluxes and forces on the membrane. In the case of heterogeneous solutions (without mechanical stirring of the solutions in the chambers), the appearance of CBL near the membrane, reduces the value of the respective fluxes and increases the value of coupling factors for the same concentrations of solute in relation to homogeneous conditions. In addition, coupling coefficients for heterogeneous conditions strongly depend on the membrane configuration.

In Configuration $\mathrm{A}$, the increase in glucose at a constant ethanol concentration at the beginning causes an increase in the coupling coefficients. In Configuration B, an increase in glucose causes a decrease in the value of coupling coefficients. The range of glucose concentrations for which the change in coupling coefficients in Configuration B is maximum is within the range similar to Configuration A of the membrane system. Analyzing the characteristics of coupling coefficients in heterogeneous conditions, we observed that for the respective characteristics in the A and B configurations of the membrane system, the respective graphs pairs (1A and 1B, 2A and $2 \mathrm{~B}$ ) intersect at a concentration of about $9.2 \mathrm{~mol} \mathrm{~m}^{-3}$. At this glucose concentration, the densities of the ternary solutions in the upper and lower chambers at the initial moment are the same. In this case, we observed the appearance of hydrodynamic instabilities that cause a disturbance of CBL diffusion reconstruction. Despite the fact that the solution densities were the same at the initial moment, the diffusion of glucose and ethanol through the membrane caused the appearance of sufficiently large and concentration gradients (and density gradients) in opposite direction to the gravitational field in the CBL areas. These gradients can cause hydrodynamic instabilities in the membrane system. 
Graphs in Figure 17 show that in the case of heterogeneous solutions (solutions not mechanically mixed-Graphs 1A and 1B, 2A and 2B), the coupling factors do not show their dependence on the configuration of the membrane system. Perhaps, because their value is very small.

Figures 17-19 show the dependences $\left(e_{i j}^{r}\right)_{r}=f\left(\bar{C}_{1}, \bar{C}_{2}=37.71 \mathrm{~mol} / \mathrm{m}^{3}\right)$ and $\left(e_{i j}\right)_{r}=f\left(\bar{C}_{1}, \bar{C}_{2}=\right.$ $\left.37.71 \mathrm{~mol} / \mathrm{m}^{3}\right),(i, j \in\{1,2,3\}$ and $r=\mathrm{A}, \mathrm{B})$ calculated on the basis of Equations (13) and (14) and data presented in Figures 14-16. Figure 17 shows that Curves 1A and 1B intersect at a point with coordinates: $\left(e_{12}^{A}\right)_{r}=\left(e_{12}^{B}\right)_{r}=0.036$ and $\bar{C}_{1}=9.18 \mathrm{~mol} / \mathrm{m}^{3}$, and Curves $2 \mathrm{~A}$ and $2 \mathrm{~B}$ - at a point with coordinates: $\left(e_{21}^{A}\right)_{r}=\left(e_{21}^{B}\right)_{r}=0.032$ and $\bar{C}_{1}=9.41 \mathrm{~mol} / \mathrm{m}^{3}$. The course of Curves $1 \mathrm{~A}, 1 \mathrm{~B}$ and 1 shows that for $\bar{C}_{1}<9.18 \mathrm{~mol} / \mathrm{m}^{3},\left(e_{12}^{B}\right)_{r}>\left(e_{12}^{A}\right)_{r}>\left(e_{12}\right)_{r}$ and for $\bar{C}_{1}>9.18 \mathrm{~mol} / \mathrm{m}^{3},\left(e_{12}^{A}\right)_{r}>\left(e_{12}^{B}\right)_{r}>\left(e_{12}\right)_{r}$. Similarly, the Curves $2 \mathrm{~A}, 2 \mathrm{~B}$ and 2 show that for $\bar{C}_{1}<9.41 \mathrm{~mol} / \mathrm{m}^{3},\left(e_{21}^{B}\right)_{r}>\left(e_{21}^{A}\right)_{r}>\left(e_{21}\right)_{r}$ and for $\bar{C}_{1}>9.41 \mathrm{~mol} / \mathrm{m}^{3}$, $\left(e_{21}^{A}\right)_{r}>\left(e_{21}^{B}\right)_{r}>\left(e_{21}\right)_{r}$. Curves $1 \mathrm{~B}$ and $2 \mathrm{~B}$ have maxima. The coordinates of the maximum of Curve $1 \mathrm{~B}$ are $\left(e_{12}^{B}\right)_{r}=0.065$ and $\bar{C}_{1}=6.54 \mathrm{~mol} / \mathrm{m}^{3}$. In turn, the coordinates of maximum of the $2 \mathrm{~B}$ curve are $\left(e_{21}^{B}\right)_{r}=0.054$ and $\bar{C}_{1}=7.23 \mathrm{~mol} / \mathrm{m}^{3}$. This means that the maximum Curve $1 \mathrm{~B}$ is shifted relative to the maximum Curve 2B vertically by $\left(r_{12}^{B}-r_{21}^{B}\right)=0.011$ and horizontally by $\Delta \bar{C}_{1}=0.69 \mathrm{~mol} / \mathrm{m}^{3}$. Curves $1 \mathrm{~B}$ and 2B coincide on the section with coordinates: $\left(e_{12}^{B}\right)_{r}=\left(e_{21}^{B}\right)_{r}=0.045$ and $\bar{C}_{1}=8.73 \mathrm{~mol} / \mathrm{m}^{3}$ and $\left(e_{12}^{B}\right)_{r}$ $=\left(e_{21}^{B}\right)_{r}=0.028$ and $\bar{C}_{1}=13.09 \mathrm{~mol} / \mathrm{m}^{3}$. For $\bar{C}_{1}<8.73 \mathrm{~mol} / \mathrm{m}^{3}$ the conditions: $\left(e_{12}^{B}\right)_{r}>\left(e_{21}^{B}\right)_{r}\left(\right.$ for $\bar{C}_{1}>$ $\left.13.09 \mathrm{~mol} / \mathrm{m}^{3}\right)$ and $\left(e_{12}^{B}\right)_{r}<\left(e_{21}^{B}\right)_{r}$ are fulfilled. Curves 1 and 2 show that the condition $\left(e_{12}\right)_{r}=\left(e_{21}\right)_{r}$ is fulfilled.

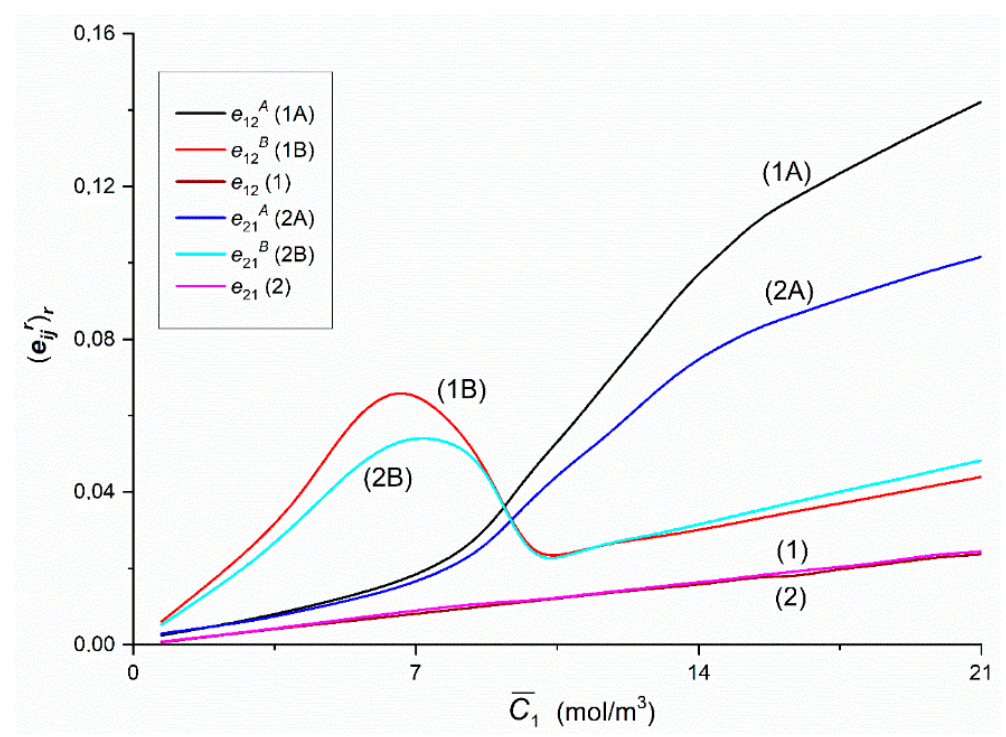

Figure 17. The $\left(e_{i j}^{r}\right)_{r}$ and $\left(e_{i j}\right)_{r}(i, j \in\{1,2\}, r=\mathrm{A}, \mathrm{B})$ coefficients as functions of glucose concentration.

Figure 18 shows that Graphs $1 \mathrm{~A}, 1 \mathrm{~B}, 2 \mathrm{~A}$ and $2 \mathrm{~B}$ intersect approximately at the point with the coordinates $\left(e_{13}^{A}\right)_{r}=\left(e_{13}^{B}\right)_{r} \approx 0.008$ and $\bar{C}_{1}=9.24 \mathrm{~mol} / \mathrm{m}^{3}$. Curves $1 \mathrm{~A}, 1 \mathrm{~B}$ and 1 show that for $\bar{C}_{1}<9.24$ $\mathrm{mol} / \mathrm{m}^{3}\left(e_{13}^{B}\right)_{r}>\left(e_{13}^{A}\right)_{r}>\left(e_{13}\right)_{r}$ and for $\bar{C}_{1}>9.24 \mathrm{~mol} / \mathrm{m}^{3}\left(e_{13}^{A}\right)_{r}>\left(e_{13}^{B}\right)_{r}>\left(e_{13}\right)_{r}$. Similarly, the course of Curves $2 \mathrm{~A}, 2 \mathrm{~B}$ and 2 shows that for $\bar{C}_{1}<9.24 \mathrm{~mol} / \mathrm{m}^{3}\left(e_{31}^{B}\right)_{r}>\left(e_{31}^{A}\right)_{r}>\left(e_{31}\right)_{r}$ and for $\bar{C}_{1}>9.24 \mathrm{~mol} / \mathrm{m}^{3}$ $\left(e_{31}^{A}\right)_{r}>\left(e_{31}^{B}\right)_{r}>\left(e_{31}\right)_{r}$. Curves $1 \mathrm{~B}$ and $2 \mathrm{~B}$ coincide for $\bar{C}_{1}>9.24 \mathrm{~mol} / \mathrm{m}^{3}$. Therefore, it can be assumed that for this concentration range $\left(e_{31}^{A}\right)_{r}>\left(e_{31}^{B}\right)_{r}>\left(e_{31}\right)_{r}$. In the other ranges $\bar{C}_{1}$ Curves 1A and 2A do not cover. This means that $\left(e_{13}^{B}\right)_{r}>\left(e_{31}^{B}\right)_{r}$ and $\left(e_{13}^{A}\right)_{r}>\left(e_{31}^{A}\right)_{r}$. Curves 1 and 2 show that the condition $\left(e_{13}\right)_{r}=\left(e_{31}\right)_{r}$. 


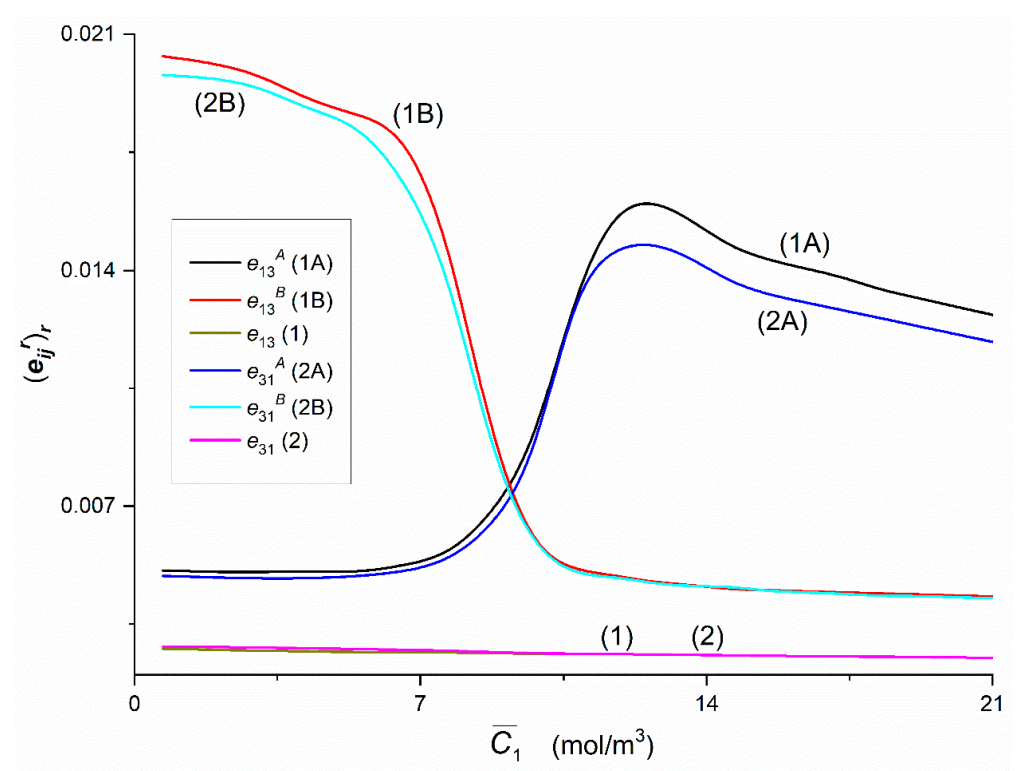

Figure 18. The $\left(e_{i j}^{r}\right)_{r}$ and $\left(e_{i j}\right)_{r}(i, j \in\{1,3\}, r=\mathrm{A}, \mathrm{B})$ coefficients as functions of glucose concentration.

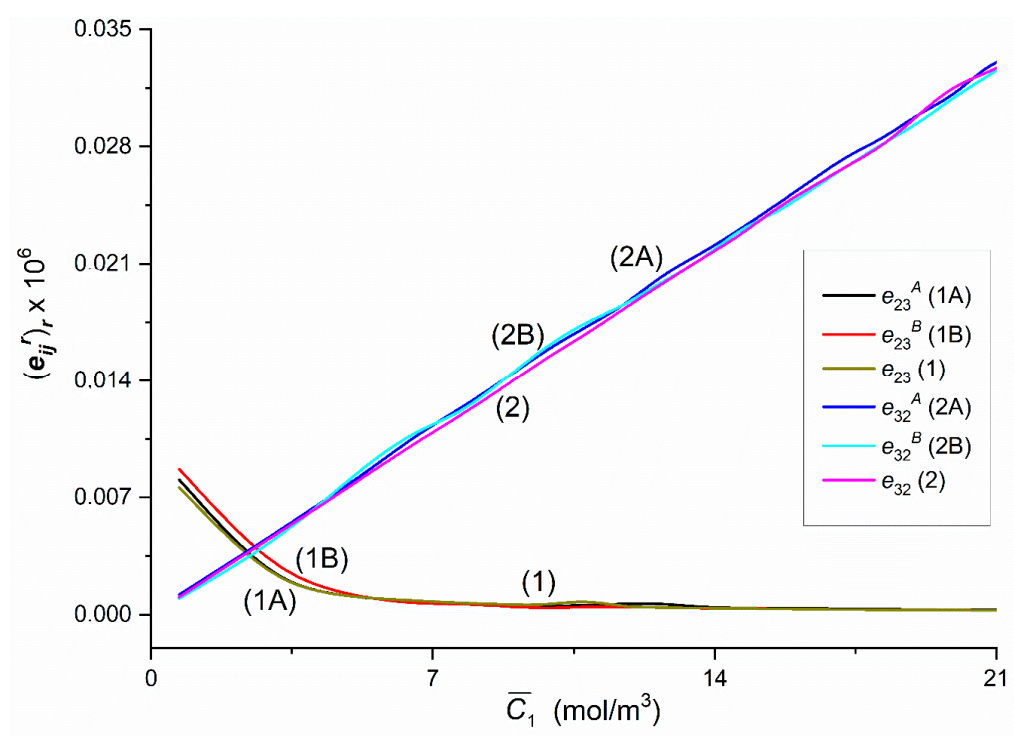

Figure 19. The $\left(e_{i j}^{r}\right)_{r}$ and $\left(e_{i j}\right)_{r}(i, j \in\{2,3\}(10 \mathrm{~b}), r=\mathrm{A}, \mathrm{B})$ coefficients as functions of glucose concentration.

From the course of Curves 1A, $1 \mathrm{~B}$ and 1 presented in Figure 19 it follows that $\left(e_{23}^{A}\right)_{r}=\left(e_{23}^{B}\right)_{r}=\left(e_{23}\right)_{r}$ and $\left(e_{32}^{A}\right)_{r}=\left(e_{32}^{B}\right)_{r}=\left(e_{32}\right)_{r}$. Curves $1,1 \mathrm{~A}$ and $1 \mathrm{~B}$ and $2,2 \mathrm{~A}$ and $2 \mathrm{~B}$ intersect at a point with coordinates $\left(e_{23}\right)_{r}=\left(e_{23}^{A}\right)_{r}=\left(e_{23}^{B}\right)_{r}=\left(e_{32}\right)_{r}=\left(e_{32}^{A}\right)_{r}=\left(e_{32}^{B}\right)_{r} \approx 0.004$ and $\bar{C}_{1}=2.57 \mathrm{~mol} / \mathrm{m}^{3}$. For $\bar{C}_{1}<2.57 \mathrm{~mol} / \mathrm{m}^{3}$, $\left(e_{23}\right)_{r}=\left(e_{23}^{A}\right)_{r}=\left(e_{23}^{B}\right)_{r}>\left(e_{32}\right)_{r}=\left(e_{32}^{A}\right)_{r}=\left(e_{32}^{B}\right)_{r}$ and for $\bar{C}_{1}>2.57 \mathrm{~mol} / \mathrm{m}^{3},\left(e_{23}\right)_{r}=\left(e_{23}^{A}\right)_{r}=\left(e_{23}^{B}\right)_{r}<\left(e_{32}\right)_{r}$ $=\left(e_{32}^{A}\right)_{r}=\left(e_{32}^{B}\right)_{r}$.

Figures 17-19 show that Kedem-Caplan relations take the form: $0.005 \leq\left(e_{12}\right)_{r}=\left(e_{21}\right)_{r} \leq 0.05$, $0.002 \leq\left(e_{12}^{A}\right)_{r} \leq 0.145,0.006 \leq\left(e_{12}^{B}\right)_{r} \leq 0.068,0.003 \leq\left(e_{21}^{A}\right)_{r} \leq 0.104,0.005 \leq\left(e_{21}^{B}\right)_{r} \leq 0.054,0.004 \leq\left(e_{13}\right)_{r}$ $=\left(e_{31}\right)_{r} \leq 0.02,0.005 \leq\left(e_{13}^{A}\right)_{r} \leq 0.016,0.004 \leq\left(e_{13}^{B}\right)_{r} \leq 0.02,0.005 \leq\left(e_{31}^{A}\right)_{r} \leq 0.015,0.04 \leq\left(e_{31}^{B}\right)_{r} \leq 0.02$, $0.003 \times 10^{-6} \leq\left(e_{23}\right)_{r}=\left(e_{23}^{A}\right)_{r}=\left(e_{23}^{B}\right)_{r} \leq 0.009 \times 10^{-6}, 0.001 \times 10^{-6} \leq\left(e_{32}\right)_{r}=\left(e_{32}^{A}\right)_{r}=\left(e_{32}^{B}\right)_{r} \leq 0.034 \times$ $10^{-6}$. Hence it follows that, $\left(e_{12}^{A}\right)_{r} \neq\left(e_{21}^{A}\right)_{r^{\prime}}\left(e_{12}^{B}\right)_{r} \neq\left(e_{21}^{B}\right)_{r^{\prime}}\left(e_{13}^{A}\right)_{r} \approx\left(e_{31}^{A}\right)_{r^{\prime}}\left(e_{13}^{B}\right)_{r}=\left(e_{31}^{B}\right)_{r^{\prime}}\left(e_{23}\right)_{r}=\left(e_{23}^{A}\right)_{r}=$ 
$\left(e_{23}^{B}\right)_{r} \neq\left(e_{32}\right)_{r}=\left(e_{32}^{A}\right)_{r}=\left(e_{32}^{B}\right)_{r}$. The values of all coupling coefficients presented in Figures 14-16 fulfill the conditions $0 \leq\left(e_{i j}\right)_{r} \leq 1,0 \leq\left(e_{i j}^{A}\right)_{r} \leq 1,0 \leq\left(e_{j i}\right)_{r} \leq 1,0 \leq\left(e_{j i}^{A}\right)_{r} \leq 1$ determined by Roy Caplan [20].

Figures 20 and 21 show the dependences $\left(Q_{R}^{r}\right)_{i j}=f\left(\bar{C}_{1}, \bar{C}_{2}=37.71 \mathrm{~mol} / \mathrm{m}^{3}\right)$ and $\left(Q_{r}\right)_{i j}=f\left(\bar{C}_{1}, \bar{C}_{2}\right.$ $\left.=37.71 \mathrm{~mol} / \mathrm{m}^{3}\right),(i, j \in\{1,2,3\}$ and $r=\mathrm{A}, \mathrm{B})$ calculated on the basis of Equations (15) and (16) and data presented in Figures 14-16. Figure 20 shows that Curves $1 \mathrm{~A}$ and $1 \mathrm{~B}$ intersect at a point with coordinates: $\left(Q_{R}^{A}\right)_{12}=\left(Q_{R}^{B}\right)_{12}=0.07$ and $\bar{C}_{1}=9.24 \mathrm{~mol} / \mathrm{m}^{3}$. The course of Curves $1 \mathrm{~A}, 1 \mathrm{~B}$ and 1 shows that for $\bar{C}_{1}<9.24 \mathrm{~mol} / \mathrm{m}^{3},\left(Q_{R}^{B}\right)_{12}>\left(Q_{R}^{A}\right)_{12}>\left(Q_{R}\right)_{12}$ and for $\bar{C}_{1}>9.24 \mathrm{~mol} / \mathrm{m}^{3},\left(Q_{R}^{A}\right)_{12}>\left(Q_{R}^{B}\right)_{12}>$ $\left(Q_{R}\right)_{12}$. Curve $1 \mathrm{~B}$ has a maximum. The coordinates of the maximum of Curve $1 \mathrm{~B}$ are $\left(Q_{R}^{B}\right)_{12}=0.12$ and $\bar{C}_{1}=6.77 \mathrm{~mol} / \mathrm{m}^{3}$. Figure 21 shows that Graphs $1 \mathrm{~A}$ and $1 \mathrm{~B}$ intersect at the point with the coordinates $\left(Q_{R}^{A}\right)_{13}=\left(Q_{R}^{B}\right)_{13}=0.015$ and $\bar{C}_{1}=9.16 \mathrm{~mol} / \mathrm{m}^{3}$. Curves $1 \mathrm{~A}, 1 \mathrm{~B}$ and 1 show that for $\bar{C}_{1}<9.24 \mathrm{~mol} / \mathrm{m}^{3}$ $\left(Q_{R}^{B}\right)_{13}>\left(Q_{R}^{A}\right)_{13}>\left(Q_{R}\right)_{13}$ and for $\bar{C}_{1}>9.24 \mathrm{~mol} / \mathrm{m}^{3}\left(Q_{R}^{A}\right)_{13}>\left(Q_{R}^{B}\right)_{13}>\left(Q_{R}\right)_{13}$. Moreover, it was shown that $\left(Q_{R}^{B}\right)_{23}=\left(Q_{R}^{A}\right)_{23}=\left(Q_{R}\right)_{23}=0.58 \times 10^{-8}=$ constant. Figures 20 and 21 show that Kedem-Caplan relations take the form: $0.01 \leq\left(Q_{R}\right)_{12} \leq 0.05,0.05 \leq\left(Q_{R}^{A}\right)_{12} \leq 0.27,0.01 \leq\left(Q_{R}^{B}\right)_{12} \leq 0.13,0.005 \leq\left(Q_{R}\right)_{13}$ $\leq 0.0055,0.008 \leq\left(Q_{R}^{A}\right)_{13} \leq 0.041,0.01 \leq\left(Q_{R}^{B}\right)_{13} \leq 0.031$. The values of all coupling coefficients presented in Figures 20 and 21 fulfill the conditions $0 \leq\left(Q_{R}\right)_{i j} \leq 1$ and $0 \leq\left(Q_{R}^{r}\right)_{i j} \leq 1$.

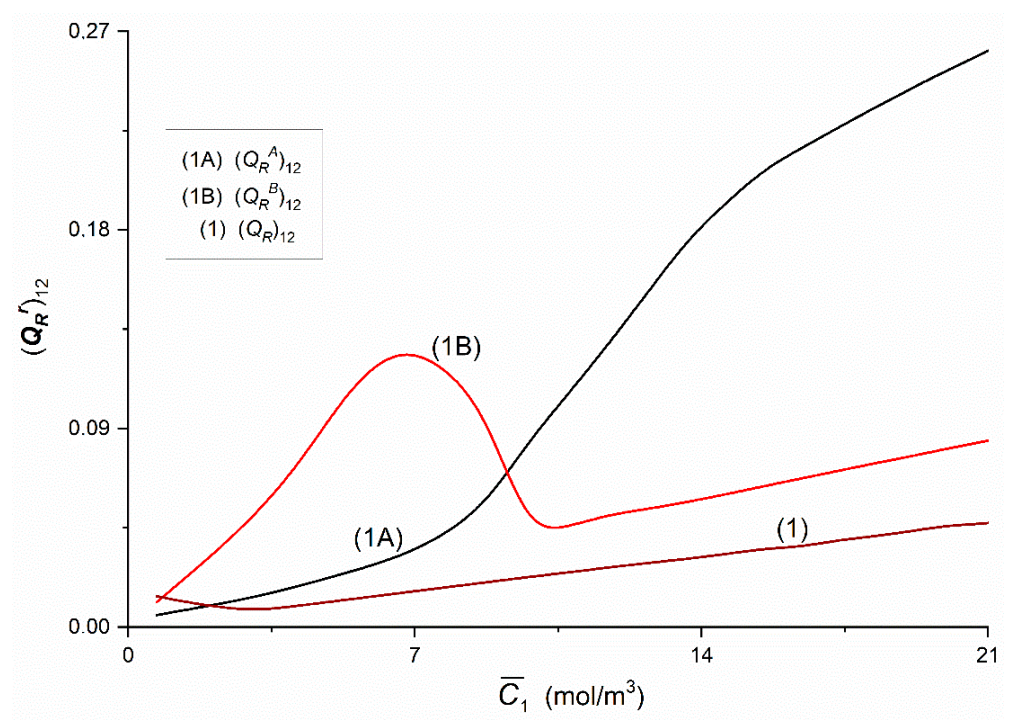

Figure 20. The $\left(Q_{R}^{r}\right)_{i j}$ and $\left(Q_{R}\right)_{i j}(i, j \in\{1,2\}, r=\mathrm{A}, \mathrm{B})$ coefficients as functions of glucose concentration.

The results of experimental research indicate that $\omega_{11}>>\omega_{12}, \omega_{22}>>\omega_{21}, \zeta_{p}^{r}=\zeta_{a 1}^{r}=\zeta_{a 2}^{r}==1, \zeta_{v 1}^{r}$ $=\zeta_{s 11}^{r}=\zeta_{s 12}^{r}=\zeta_{1}^{r}$ and $\zeta_{v 2}^{r}=\zeta_{s 22}^{r}=\zeta_{s 21}^{r}=\zeta_{2}^{r}(\mathrm{r}=\mathrm{A}, \mathrm{B})$. By accepting the above conditions and that $\zeta_{1}^{r} \approx$ $\zeta_{2}^{r}=\zeta^{r}$. Given this condition, and Equations (5), (9) and (10), we can write:

$$
\begin{gathered}
\xi_{11}=\frac{\zeta^{A}-\zeta^{B}}{\zeta^{A} \zeta^{B}} \frac{L_{p}\left[\bar{C}_{1} \omega_{22}\left(1-\sigma_{1}\right)+\bar{C}_{2} \omega_{11}\left(1-\sigma_{2}\right)\right]}{\omega_{11} \omega_{22}+L_{p}\left[\omega_{22} \bar{C}_{1}\left(1-\sigma_{1}\right)^{2}+\omega_{11} \bar{C}_{2}\left(1-\sigma_{2}\right)^{2}\right]} \\
\xi_{12}=\frac{\zeta^{A}-\zeta^{B}}{\zeta^{A} \zeta^{B}} \frac{1}{\left(1-\sigma_{1}\right)} \\
\xi_{13}=\frac{\zeta^{A}-\zeta^{B}}{\zeta^{A} \zeta^{B}} \frac{1}{\left(1-\sigma_{2}\right)} \\
\xi_{21}=-\frac{\zeta^{A}-\zeta^{B}}{\zeta^{A} \zeta^{B}}=\xi_{31}=-\xi_{22}=-\xi_{23}=-\xi_{32}=-\xi_{33}
\end{gathered}
$$




$$
\xi_{\text {det }}=\frac{\left(\zeta^{A}\right)^{2}-\left(\zeta^{B}\right)^{2}}{\left(\zeta^{A}\right)^{2}\left(\zeta^{B}\right)^{2}}
$$

Equations (17)-(20) contain the factor $\left(\zeta_{1}^{A}-\zeta_{1}^{B}\right)\left(\zeta_{1}^{A} \zeta_{1}^{B}\right)^{-1}$ and Equation (22)-the factor $\left[\left(\zeta^{A}\right)^{2}-\left(\zeta^{B}\right)^{2}\right]\left[\left(\zeta^{A}\right)^{2}\left(\zeta^{B}\right)^{2}\right]^{-1}$. This factor, using Equation (1) can be written in a form containing the thickness of CBLs. To simplify the accounts, using the conditions $\left(D_{i j}^{r}\right)_{l}=\left(D_{i j}^{r}\right)_{h}=D_{i j}$ and $\delta_{h}^{r}=\delta_{l}^{r}=$ $\delta^{r}$, we write the Equation (1) in the form:

$$
\zeta^{r}=\frac{D_{i j}}{D_{i j}+2 R T \omega_{i j} \delta^{r}}
$$

Using Equation (22) we can write:

$$
\begin{gathered}
\frac{\zeta^{A}-\zeta^{B}}{\zeta^{A} \zeta^{B}}=\frac{2 R T \omega_{i j}\left(\delta^{B}-\delta^{A}\right)}{D_{i j}} \\
\frac{\left(\zeta^{A}\right)^{2}-\left(\zeta^{B}\right)^{2}}{\left(\zeta^{A}\right)^{2}\left(\zeta^{B}\right)^{2}}=\frac{4 R T \omega_{i j}}{D_{i j^{2}}}\left\{D_{i j}\left(\delta^{B}-\delta^{A}\right)+R T \omega_{i j}\left[\left(\delta^{B}\right)^{2}-\left(\delta^{A}\right)^{2}\right]\right\}
\end{gathered}
$$

From all the foregoing considerations, it is clear that coefficients $\xi_{i j}\left(i, j \in\{1,2,3\}\right.$ and $\xi_{\text {det }}$ are measures of the natural convection effect. If the conditions $\xi_{i j}<0$ and $\xi_{\text {det }}<0$ are fulfilled, fluxes of natural convection in single-membrane system are directed vertically upwards. In turn, for coefficients $\xi_{i j}>0$ and $\xi_{\text {det }}>0$, the fluxes are directed vertically downwards. Zeroing of the coefficients ( $\xi_{i j}=0$ and $\xi_{\text {det }}=0$ ) means that the system is in the critical point where the flux turns its direction from vertically upwards to vertically downwards. In this point, the structure of layers lose its stability, but natural convection does not have precise turn yet, what means that the membrane system is not sensitive to changes in the gravitational field. This is shown by dependencies $\xi_{i j}=f\left(\bar{C}_{1}, \bar{C}_{2}=37.71 \mathrm{~mol} / \mathrm{m}^{3}\right),(i, j$ $\in\{1,2,3\}$ and $\xi_{\text {det }}=f\left(\bar{C}_{1}, \bar{C}_{2}=37.71 \mathrm{~mol} / \mathrm{m}^{3}\right)$, presented in Figures $11-13$ as well as interferograms presented in the previous publication $[37,38]$. Hydrodynamic stability in the membrane system is controlled by the concentration Rayleigh number [34-38]. The Rayleigh number value depends on the concentration of solutions separated by the membrane [34,35]. For the points where $\xi_{i j}=0$ and $\xi_{\text {det }}=$ $0,(i, j \in\{1,2,3\})$ the critical value of concentration Rayleigh number $\left(R_{C}\right)$ can be specified.

For example, we will consider Equations (20) and (23) and Figures 2 and 3 for the $\xi_{22}$ coefficient. This equation can be written as $\xi_{22}=2 R T \omega_{11}\left(\delta^{B}-\delta^{A}\right) D_{11}{ }^{-1}$. It is drawn from the equation and Figure 2 that if $\xi_{22}=0$, then $\zeta_{1}^{A}=\zeta_{1}^{B}=0.234$. From the equation, it becomes apparent that if $\xi_{22}$ $=0$, then $\delta^{A}=\delta^{B}$. The values of $\delta^{A}$ and $\delta^{B}$ can be determined by laser interferometry [35-38] or volume flux measurements [34]. Figure 3 presents the dependences $\delta^{r}=f\left(\rho_{h}-\rho_{l}\right)$ obtained by converting the dependence $\zeta_{i}^{r}=f\left(\bar{C}_{1}, \bar{C}_{2}=\right.$ const.) shown in Figure 3 , with the help of equations $\delta^{r}=D_{i j}\left(1-\zeta_{i}^{r}\right)\left(2 R T \omega_{i j} \zeta_{i}^{r}\right)^{-1}$ and $\rho_{h}-\rho_{l}=\left(\partial \rho / \partial C_{1}\right)\left(C_{1 h}-C_{1 l}\right)+\left(\partial \rho / \partial C_{2}\right)\left(C_{2 h}-C_{2 l}\right)$. From this figure it follows that $\delta^{A}=\delta^{B} \approx 1.3 \times 10^{-3} \mathrm{~m}$ for $\rho_{h}-\rho_{l}=0.046 \mathrm{~kg} / \mathrm{m}^{3}$.

Let us consider the dependency $\xi_{22}=f\left(\bar{C}_{1}, \bar{C}_{2}=37.71 \mathrm{~mol} / \mathrm{m}^{3}\right)$ shown in the Figure 12. It results from the figure that $\xi_{22}=0$ for $\bar{C}_{1}=9.24 \mathrm{~mol} / \mathrm{m}^{3}$ and $\bar{C}_{2}=37.71 \mathrm{~mol} / \mathrm{m}^{3}$. It should be pointed out that $\bar{C}_{1}=9.24 \mathrm{~mol} / \mathrm{m}^{3}$ if $C_{1 h}=33.44 \mathrm{~mol} / \mathrm{m}^{3}$ and $C_{1 l}=1 \mathrm{~mol} \mathrm{~m}{ }^{-3}$ while $\bar{C}_{2}=37.71 \mathrm{~mol} / \mathrm{m}^{3}$, for $C_{2 h}=201$ $\mathrm{mol} / \mathrm{m}^{3}$ and $C_{2 l}=1 \mathrm{~mol} / \mathrm{m}^{3}$. Therefore, consisting solution density amounts to $998.3 \mathrm{~kg} / \mathrm{m}^{3}$. In turn, kinematic viscosity of this solution is equal to $v=1.063 \times 10^{-6} \mathrm{~m}^{2} / \mathrm{s}$. Density difference of solutions located in the Compartments (h) and (l) calculated on the basis of equation $\rho_{h}-\rho_{l}=\left(\partial \rho / \partial C_{1}\right)\left(C_{1 h}\right.$ $\left.-C_{1 l}\right)+\left(\partial \rho / \partial C_{2}\right)\left(C_{2 h}-C_{2 l}\right)$, where $\left(\partial \rho / \partial C_{1}\right)=0.06 \mathrm{~kg} / \mathrm{mol},\left(\partial \rho / \partial C_{2}\right)=-0.0095 \mathrm{~kg} / \mathrm{mol}$, amounts to $\rho_{h}-\rho_{l}=0.046 \mathrm{~kg} / \mathrm{m}^{3}$. Taking these data into consideration, as well as $D_{11}=0.69 \times 10^{-9} \mathrm{~m}^{2} / \mathrm{s}, g=$ 
$9.81 \mathrm{~m} / \mathrm{s}^{2}, \omega_{11}=0.8 \times 10^{-9} \mathrm{~mol} / \mathrm{Ns}, \delta=1.3 \times 10^{-3} \mathrm{~m}$ in the expression for the concentration Rayleigh number $R_{C}=\left[g\left(\rho_{h}-\rho_{l}\right)(\delta)^{3}\right]\left(\rho_{h} v_{h} D_{11}\right)^{-1}[29,30]$, we get $R_{C}=1353.1$. This value corresponds to the $\left(R_{C}\right)_{\text {crit. }}=1100.6$, obtained for the case of the rigid membrane surface and the free liquid interior (rigid-free borders) [44,45]. For electrolysis occurring in a cell containing electrodes placed in parallel in horizontal planes, the critical Rayleigh number depends strongly on the distance between these electrodes and for amperostatic conditions takes the values in the range of $R_{C}=1070 \div 1540$ [46]. In turn, for potentiostatic conditions $R_{C}$ takes the values in the range of $R_{C}=763.3 \div 1351$ [47].

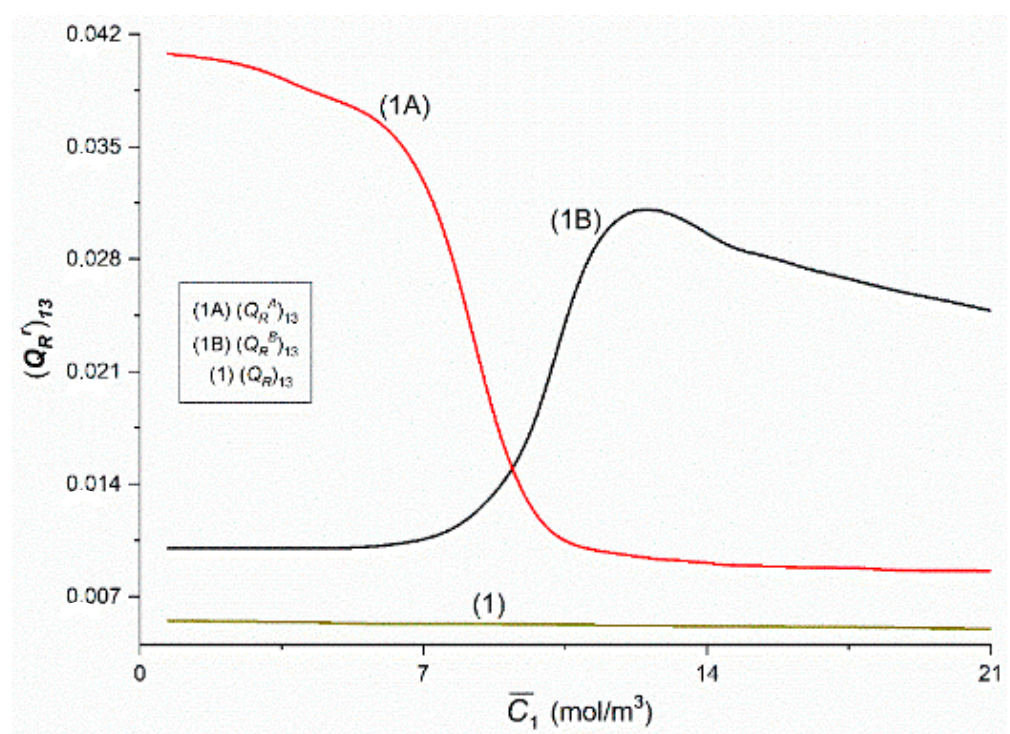

Figure 21. The $\left(Q_{R}^{r}\right)_{i j}$ and $\left(Q_{R}\right)_{i j}(i, j \in\{1,3\}, r=\mathrm{A}, \mathrm{B})$ coefficients as functions of glucose concentration.

\section{Conclusions}

From the above presented studies, the following results are obtained:

1. In order to describe transport processes of ternary solutions of nonelectrolytes through horizontally oriented membrane, nine Peusner's coefficients should be calculated $R_{i j}^{r}(i, j \in\{1,2,3\}, r=\mathrm{A}, \mathrm{B})$ and the determinant of the matrix of these coefficients is $\operatorname{det}\left[R^{r}\right]=R_{d e t}^{r}$. For the Nephrophan membrane and aqueous solutions of glucose and ethanol, the values of coefficients $R_{i j}^{r}(i, j \in$ $\{1,2,3\}, r=\mathrm{A}, \mathrm{B})$ and $R_{d e t}^{r}$ are dependent on concentration solutions and configuration of the membrane system. For $i \neq j$ these coefficients fulfill the relations $R_{i j}^{r} \neq R_{j i}^{r}$.

2. Concentration dependencies of coefficients $\xi_{i j}=\left(R_{i j}^{A}-R_{i j}^{B}\right) / R_{i j}=f\left(\bar{C}_{1}, \bar{C}_{2}=37.71 \mathrm{~mol} / \mathrm{m}^{3}\right)$ and $\left.\xi_{\text {det }}=\left(\operatorname{det}\left[R^{A}\right]-\operatorname{det}\left[R^{B}\right]\right) / \operatorname{det}[R]\right)=f\left(\bar{C}_{1}, \bar{C}_{2}=37.71 \mathrm{~mol} / \mathrm{m}^{3}\right)$ facilitate estimation of natural convection direction: for $\xi_{i j}<0$, natural convection is directed vertically upwards and for $\xi_{i j}>$ 0 -vertically downwards. The value of coefficients $\xi_{i j}$ and $\xi_{\text {det }}\left(\xi_{i j}<0, \xi_{\text {det }}<0, \xi_{i j}=0, \xi_{\text {det }}=\right.$ $0, \xi_{i j}>0$ or $\xi_{\text {det }}>0$ ) shows the influence of concentration polarization and natural convection on the membrane transport. For $\xi_{i j}=0$ the critical value of the concentration Rayleigh number $\left(R_{C}\right)$ can be estimated, for the point where convective stream changes its direction from vertical upwards into vertical downwards. The $R_{C}$ value estimated in this paper for the considered case amounts to $\left(R_{C}\right)_{\text {crit. }}=1353.1$.

3. For $(i, j \in\{1,2\}, r=\mathrm{A}, \mathrm{B})$ the coupling $\left(r_{i j}^{r}\right),\left(Q_{R}^{r}\right)_{i j}$ and energy conversion $\left(e_{i j}^{r}\right)_{r}$ Coefficients depend on the concentration of homogeneous solutions and in concentration polarization conditions-on the concentration of solutions and the configuration of the membrane system. For $(i, j \in\{1,3\}, r=$ A, B) these coefficients in concentration polarization conditions depend and in homogeneous solutions do not depend on the concentration of solutions and the configuration of the membrane system. The crisscrosses of suitable A and B characteristics are observed at a glucose concentration 
$\bar{C}_{1}=9.24 \mathrm{~mol} \mathrm{~m}^{-3}$. For $(i, j \in\{2,3\}, r=\mathrm{A}, \mathrm{B})$ the coefficients $\left(r_{i j}^{r}\right)$ and $\left(e_{i j}^{r}\right)_{r}$ depend on the concentration of homogeneous solutions and in concentration polarization conditions and do not depend on the configuration of the membrane system. The crisscrosses of suitable A and B characteristics are observed at a glucose concentration $\bar{C}_{1} \approx 2.5 \mathrm{~mol} \mathrm{~m}^{-3}$. The $\left(Q_{R}^{r}\right)_{i j}$ coefficient is independent of the concentration and configuration of the membrane system.

4. Curves marked with a number and the letters A or B are evidence that there are transition points associated with the change in the nature of membrane transport from osmotic-diffusion to osmotic-diffusion-convective or vice versa. This means that in Configuration A, we have a transition from convective to convective, and in Configuration B-from convective to non-convective. These transitions are a pseudo-phase transition.

5. The presented equations are a new research tool for membrane transport and the influence of gravity field on this transport.

Author Contributions: Conceptualization, K.M.B. and A.Ś.; methodology, K.M.B and A.Ś.; calculation and investigation: K.M.B., A.Ś.; writing—original draft preparation, K.M.B., A.S., S.M. and W.M.B.; writing—review and editing K.M.B., A.Ś., S.G. and W.M.B. All authors have read and approved the final manuscript.

Funding: This research received no external funding.

Acknowledgments: We would like to thank our astoundingly supportive research team and for those who have touched our science paths.

Conflicts of Interest: The authors declare no conflict of interest.

\section{References}

1. Kondepudi, D. Introduction to Modern Thermodynamics; John Wiley \& Sons: Chichester, UK, 2008; ISBN 978-0-470-01598-8.

2. Baker, R. Membrane Technology and Application; John Wiley \& Sons: New York, NY, USA, 2012; ISBN 978-0-470-74372-0.

3. Uragami, T. Science and Technology of Separation Membranes; John Wiley \& Sons: Chichester, UK, 2017; ISBN 978-1-11893-254-4.

4. Vafai, K. Porous Media: Applications in Biological Systems and Biotechnology; CRC Press: Boca Raton, FL, USA, 2011; ISBN 978-1-4200-6541-1.

5. Plawsky, J.L. Transport Phenomena Fundamentals; CRC Press: Boca Raton, FL, USA, 2020; ISBN 978-1-138-08056-0.

6. Hoogendoorn, A.; van Kasteren, H. Transportation Biofules: Pathways for Production; Royal Society of Chemistry: London, UK, 2020; ISBN 978-1-78801-504-2.

7. Speight, J.G. Natural Water Remediation: Chemistry and Technology; Elsevier: Amsterdam, The Netherlands, 2019; ISBN 978-0-12-803810-9.

8. Dermirel, Y. Nonequilibrium Thermodynamics: Transport and Rate Processes in Physical, Chemical and Biological Systems; Elsevier: Amsterdam, The Netherlands, 2007; pp. 275-540. ISBN 978-0-444-53079-0.

9. Nikonenko, V.V.; Kovalenko, A.V.; Urtenov, M.K.; Pismenskaya, N.D.; Han, J.; Sistet, P.; Pourcelly, G. Desalination at Overlimitinng Currents: State-Of-Theart and Perspectives. Desalination 2014, 342, 85-106. [CrossRef]

10. Kedem, O.; Katchalsky, A. Thermodynamics Analysis of the Permeability of Biological Membranes to Non-Electrolytes. Biochim. Biophys. Acta 1958, 27, 229-246. [CrossRef]

11. Katchalsky, A.; Curran, P.F. Nonequilibrium Thermodynamics in Biophysics; Harvard University Press: Cambridge, MA, USA, 1965; ISBN 9780674494121.

12. Kargol, A.; Kargol, M.; Przestalski, S. The Kedem-Katchalsky Equations as Applied for Describing Substance Transport Across Biological Membranes. Cell. Mol. Biol. Lett. 1997, 2, 117-124.

13. Kargol, A. A Mechanistic Model of Transport Processes in Porous Membranes Generated by Osmotic and Hydrostatic Pressure. J. Membr. Sci. 2001, 191, 61-69. [CrossRef]

14. Kargol, M.; Kargol, A. Mechanistic Formalism for Membrane Transport Generated by Osmotic and Mechanical Pressure. Gen. Physiol. Biophys. 2003, 22, 51-68. [PubMed] 
15. Peusner, L. Studies in Network Thermodynamics; Elsevier: Amsterdam, The Netherlands, 1986; pp. 144-342. ISBN 0-444-42580-2.

16. Elmoazzen, H.Y.; Elliot, J.A.W.; McGann, L.E. Osmotic Transport across Cell Membranes in Nondilute Solutions: A New Nondilute Solute Transport Equation. Biophys. J. 2009, 96, 2559-2571. [CrossRef] [PubMed]

17. Cheng, X.; Pinsky, P.M. The Balance of Fluid and Osmotic Pressures across Active Biological Membranes with Application to the Corneal Endothelium. PLoS ONE 2015, 10, e0145422. [CrossRef]

18. Cardoso, S.S.S.; Cartwright, J.H.E. Dynamic of Osmosis in a Porous Medium. R. Soc. Open Sci. 2014, 1, 140352. [CrossRef]

19. Kedem, O.; Caplan, S.R. Degree of Coupling and Its Relation to Efficiency of Energy Conversion. Trans. Faraday Soc. 1965, 61, 1897-1911. [CrossRef]

20. Caplan, S.R. The Degree of Coupling and Its Relation to Efficiency of Energy Conversion in Multiple-Flow Systems. J. Theor. Biol. 1965, 10, 209-235. [CrossRef]

21. Peusner, L. Hierarchies of Irreversible Energy Conversion Systems: A Network Thermodynamics Approach. I. Linear Steady State without Storage. J. Theor. Biol. 1983, 10, 27-39. [CrossRef]

22. Peusner, L. Hierarchies of Irreversible Energy Conversion Systems II. Network Derivation of Linear Transport Equations. J. Theor. Biol. 1985, 115, 319-335. [CrossRef]

23. Peusner, L. The Principles of Network Thermodynamics: Theory and Biophysical Applications. Ph.D. Thesis, Harvard University, Cambridge, MA, USA, 1970.

24. Oster, G.; Perelson, A.; Katchalsky, A. Network Thermodynamics. Nature 1971, 234, 393-399. [CrossRef]

25. Ślęzak, A.; Grzegorczyn, S.; Batko, K.M. Resistance Coefficients of Polymer Membrane with Concentration Polarization. Transp. Porous Media 2012, 95, 151-170. [CrossRef]

26. Batko, K.M.; Ślęzak-Prochazka, I.; Grzegorczyn, S.; Ślęzak, A. Membrane Transport in Concentration Polarization Conditions: Network Thermodynamics Model Equations. J. Porous Media 2014, 17, 573-586. [CrossRef]

27. Batko, K.M.; Ślęzak-Prochazka, I.; Ślęzak, A. Network Hybrid Form of the Kedem-Katchalsky Equations for Non-Homogenous Binary Non-Electrolyte Solutions: Evaluation of $P_{i j}$ * Peusner's Tensor Coefficients. Transp. Porous Media 2015, 106, 1-20. [CrossRef]

28. Ślęzak-Prochazka, I.; Batko, K.M.; Wasik, S.; Ślęzak, A. $H^{*}$ Peusner's Form of the Kedem-Katchalsky Equations Fon On-Homogeneous Non-Electrolyte Binary Solutions. Transp. Porous Media 2016, 111, 457-477. [CrossRef]

29. Ślęzak, A.; Dworecki, K.; Anderson, J.A. Gravitational Effects on Transmembrane Flux: The Rayleigh-Taylor Convective Instability. J. Membr. Sci. 1985, 23, 71-81. [CrossRef]

30. Ślęzak, A.; Grzegorczyn, S.; Jasik-Ślęzak, J.; Michalska-Małecka, K. Natural Convection as an Asymmetrical Factor of the Transport through Porous Membrane. Transp. Porous Media 2010, 84, 685-698. [CrossRef]

31. Schlichting, H.; Gersten, K. Boundary Layers Theory; Springer: Berlin/Heidelberg, Germany, 2000; ISBN 978-3-642-85831-4.

32. Barry, P.H.; Diamond, J.M. Effects of Unstirred Layers on Membrane Phenomena. Physiol. Rev. 1984, 64, 763-872. [CrossRef]

33. Ślęzak, A. Irreversible Thermodynamic Model Equations of the Transport across a Horizontally Mounted Membrane. Biophys. Chem. 1989, 34, 91-102. [CrossRef]

34. Jasik-Ślęzak, J.; Olszówka, K.M.; Ślęzak, A. Estimation of Thickness of Concentration Boundary Layers by oSmotic Volume Flux Determination. Gen. Physiol. Biophys. 2011, 30, 186-195. [CrossRef] [PubMed]

35. Dworecki, K.; Ślęzak, A.; Ornal-Wąsik, B.; Wąsik, S. Effect of Hydrodynamic Instabilities on Solute Transport in Membrane System. J. Membr. Sci. 2005, 265, 94-100. [CrossRef]

36. Ślęzak, A.; Dworecki, K.; Jasik-Ślęzak, J.; Wassik, J. Method to Determine the Practical Concentration Rayleigh Number in Isothermal Passive Membrane Transport Processes. Desalination 2004, 168, 397-412. [CrossRef]

37. Ślęzak, A.; Dworecki, K.; Ślęzak, I.H.; Wąsik, S. Permeability Coefficient Model Equations of the Complex: Membrane-Concentration Boundary Layers for Ternary Nonelectrolyte Solutions. J. Membr. Sci. 2005, 267, 50-57. [CrossRef]

38. Dworecki, K.; Wąsik, S.; Ślęzak, A. Temporal and Spatial Structure of the Concentration Boundary Layers in Membrane System. Physica A 2003, 326, 360-369. [CrossRef]

39. Levitt, M.D.; Strocchi, D.; Levitt, G. Human Jejunum Unstirred Layer: Evidence for Efficient Luminal Stirring. Am. J. Physiol. 1989, 93, 631-647. 
40. Shibayama, T.; Morales, M.; Zhang, X.; Martínez-Guerrero, L.J.; Berteloot, A.; Secomb, T.W.; Wright, S.H. Unstirred Water Layers and the kinEtics of Organic Cation Transport. Pharm. Res. 2015, 32, 2937-2949. [CrossRef]

41. Winne, D. Unstirred Layer, Source of Biased Michaelis Constant in Membrane Transport. Biochem. Biophys. Acta 1973, 298, 27-31. [CrossRef]

42. Batko, K.; Ślęzak, A. Membrane Transport of Nonelectrolyte Solutions in Concentration Polarization Conditions: $\mathrm{H}^{\mathrm{r}}$ Form of the Kedem-Katchalsky-Peusner Equations. Int. J. Chem. Eng. 2019, 2019. ID5629259. [CrossRef]

43. Ślęzak, A.; Grzegorczyn, S.; Batko, K.M.; Bajdur, W.M.; Makuła-Włodarczyk, M. Applicability of the $L_{r}$ Form of the Kedem-Katchalsky-Peusner Equations for Membrane Transport in Water Purification Technology. Des. Water Treat. 2020. in print.

44. Lebon, G.; Jou, D.; Casas-Vasquez, J. Understanding Non-Equilibrium Thermodynamics. Foundations, Applications, Frontiers; Springer: Berlin/Heidelberg, Germany, 2008. [CrossRef]

45. Batko, K.M.; Slęzak, A.; Bajdur, W.M. The Role of Gravity in the Evolution of the Concentration Field in the Electrochemical Membrane Cell. Entropy 2020, 22, 680. [CrossRef]

46. Baranowski, B.; Kawczyński, A. Experimental Determination of the Critical Rayleigh Number Inelectrolyte Solutions with Concentration Polarization. Electrochim. Acta 1972, 17, 695-699. [CrossRef]

47. Baranowski, B. The Electrochemical Analogon of the Benard Instability Studied at Isothermal and Potentiostatic Conditions. J. Non-Equilib. Thermodyn. 1980, 5, 67-72. [CrossRef]

(C) 2020 by the authors. Licensee MDPI, Basel, Switzerland. This article is an open access article distributed under the terms and conditions of the Creative Commons Attribution (CC BY) license (http://creativecommons.org/licenses/by/4.0/). 